\title{
Hitting probabilities for systems of non-linear stochastic heat equations with multiplicative noise
}

\author{
Robert C. Dalang ${ }^{1,4}$, Davar Khoshnevisan ${ }^{2,5}$, and Eulalia Nualart ${ }^{3}$
}

\begin{abstract}
We consider a system of $d$ non-linear stochastic heat equations in spatial dimension 1 driven by $d$-dimensional space-time white noise. The non-linearities appear both as additive drift terms and as multipliers of the noise. Using techniques of Malliavin calculus, we establish upper and lower bounds on the one-point density of the solution $u(t, x)$, and upper bounds of Gaussian-type on the two-point density of $(u(s, y), u(t, x))$. In particular, this estimate quantifies how this density degenerates as $(s, y) \rightarrow(t, x)$. From these results, we deduce upper and lower bounds on hitting probabilities of the process $\{u(t, x)\}_{t \in \mathbb{R}_{+}, x \in[0,1]}$, in terms of respectively Hausdorff measure and Newtonian capacity. These estimates make it possible to show that points are polar when $d \geq 7$ and are not polar when $d \leq 5$. We also show that the Hausdorff dimension of the range of the process is 6 when $d>6$, and give analogous results for the processes $t \mapsto u(t, x)$ and $x \mapsto u(t, x)$. Finally, we obtain the values of the Hausdorff dimensions of the level sets of these processes.
\end{abstract}

AMS 2000 subject classifications: Primary: 60H15, 60J45; Secondary: 60H07, 60G60.

Key words and phrases. Hitting probabilities, stochastic heat equation, space-time white noise, Malliavin calculus.

\footnotetext{
${ }^{1}$ Institut de Mathématiques, Ecole Polytechnique Fédérale de Lausanne, Station 8, CH-1015 Lausanne, Switzerland. robert.dalang@epfl.ch

${ }^{2}$ Department of Mathematics, The University of Utah, 155 S. 1400 E. Salt Lake City, UT 84112-0090, USA. davar@math.utah.edu

${ }^{3}$ Institut Galilée, Université Paris 13, 93430 Villetaneuse, France. nualart@math.univ-paris13.fr

${ }^{4}$ Supported in part by the Swiss National Foundation for Scientific Research.

${ }^{5}$ Research supported in part by a grant from the US National Science Foundation.
} 


\section{Introduction and main results}

Consider the following system of non-linear stochastic partial differential equations (spde's)

$$
\frac{\partial u_{i}}{\partial t}(t, x)=\frac{\partial^{2} u_{i}}{\partial x^{2}}(t, x)+\sum_{j=1}^{d} \sigma_{i, j}(u(t, x)) \dot{W}^{j}(t, x)+b_{i}(u(t, x)),
$$

for $1 \leq i \leq d, t \in[0, T]$, and $x \in[0,1]$, where $u:=\left(u_{1}, \ldots, u_{d}\right)$, with initial conditions $u(0, x)=0$ for all $x \in[0,1]$, and Neumann boundary conditions

$$
\frac{\partial u_{i}}{\partial x}(t, 0)=\frac{\partial u_{i}}{\partial x}(t, 1)=0, \quad 0 \leq t \leq T
$$

Here, $\dot{W}:=\left(\dot{W}^{1}, \ldots, \dot{W}^{d}\right)$ is a vector of $d$ independent space-time white noises on $[0, T] \times$ $[0,1]$. For all $1 \leq i, j \leq d, b_{i}, \sigma_{i j}: \mathbb{R}^{d} \rightarrow \mathbb{R}$ are globally Lipschitz functions. We set $b=\left(b_{i}\right)$, $\sigma=\left(\sigma_{i j}\right)$. Equation (1.1) is formal: the rigorous formulation of WALSH [W86] will be recalled in Section 2.

The objective of this paper is to develop a potential theory for the $\mathbb{R}^{d}$-valued process $u=(u(t, x), t \geq 0, x \in(0,1))$. In particular, given $A \subset \mathbb{R}^{d}$, we want to determine whether the process $u$ visits (or hits) $A$ with positive probability.

The only potential-theoretic result that we are aware of for systems of non-linear spde's with multiplicative noise ( $\sigma$ non-constant) is DALANG AND NUALART [DN04], who study the case of the reduced hyperbolic spde on $\mathbb{R}_{+}^{2}$ (essentially equivalent to the wave equation in spatial dimension 1):

$$
\frac{\partial^{2} X_{t}^{i}}{\partial t_{1} \partial t_{2}}=\sum_{j=1}^{d} \sigma_{i, j}\left(X_{t}\right) \frac{\partial^{2} W_{t}^{j}}{\partial t_{1} \partial t_{2}}+b_{i}\left(X_{t}\right)
$$

where $t=\left(t_{1}, t_{2}\right) \in \mathbb{R}_{+}^{2}$, and $X_{t}^{i}=0$ if $t_{1} t_{2}=0$, for all $1 \leq i \leq d$. There, Dalang and Nualart used Malliavin calculus to show that the solution $\left(X_{t}\right)$ of this spde satisfies

$$
K^{-1} \operatorname{Cap}_{d-4}(A) \leq \mathrm{P}\left\{\exists t \in[a, b]^{2}: X_{t} \in A\right\} \leq K_{\operatorname{Cap}_{d-4}}(A),
$$

where $\mathrm{Cap}_{\beta}$ denotes the capacity with respect to the Newtonian $\beta$-kernel $\mathrm{K}_{\beta}(\cdot)$ (see (1.6)). This result, particularly the upper bound, relies heavily on properties of the underlying two-parameter filtration and uses Cairoli's maximal inequality for two-parameter processes.

Hitting probabilities for systems of linear heat equations have been obtained in MUELLER AND TRIBE [MT03]. For systems of non-linear stochastic heat equations with additive noise, that is, $\sigma$ in (1.1) is a constant matrix, so (1.1) becomes

$$
\frac{\partial u_{i}}{\partial t}(t, x)=\frac{\partial^{2} u_{i}}{\partial x^{2}}(t, x)+\sum_{j=1}^{d} \sigma_{i, j} \dot{W}^{j}(t, x)+b_{i}(u(t, x)),
$$

estimates on hitting probabilities have been obtained in Dalang, Khoshnevisan And NuALART [DKN07]. That paper develops some general results that lead to upper and lower bounds on hitting probabilities for continuous two-parameter random fields, and then uses 
these, together with a careful analysis of the linear equation $\left(b \equiv 0, \sigma \equiv I_{d}\right.$, where $I_{d}$ denotes the $d \times d$ identity matrix) and Girsanov's theorem, to deduce bounds on hitting probabilities for the solution to (1.3).

In this paper, we make use of the general results of [DKN07], but then, in order to handle the solution of (1.1), we use a very different approach. Indeed, the results of [DKN07] require in particular information about the probability density function $p_{t, x}$ of the random vector $u(t, x)$. In the case of multiplicative noise, estimates on $p_{t, x}$ can be obtained via Malliavin calculus.

We refer in particular on the results of BALLY AND PARDOux [BP98], who used Malliavin calculus in the case $d=1$ to prove that for any $t>0, k \in \mathbb{N}$ and $0 \leq x_{1}<\cdots<x_{k} \leq 1$, the law of $\left(u\left(t, x_{1}\right), \ldots, u\left(t, x_{k}\right)\right)$ is absolutely continuous with respect to Lebesgue measure, with a smooth and strictly positive density on $\{\sigma \neq 0\}^{k}$, provided $\sigma$ and $b$ are infinitely differentiable functions which are bounded together with their derivatives of all orders. A Gaussian-type lower bound for this density is established by KoHATSU-HigA [K03] under a uniform ellipticity condition. MORIEn [M98] showed that the density function is also Hölder-continuous as a function of $(t, x)$.

In this paper, we shall use techniques of Malliavin calculus to establish the following theorem. Let $p_{t, x}(z)$ denote the probability density function of the $\mathbb{R}^{d}$-valued random vector $u(t, x)=\left(u_{1}(t, x), \ldots, u_{d}(t, x)\right)$ and for $(s, y) \neq(t, x)$, let $p_{s, y ; t, x}\left(z_{1}, z_{2}\right)$ denote the joint density of the $\mathbb{R}^{2 d}$-valued random vector

$$
(u(s, y), u(t, x))=\left(u_{1}(s, y), \ldots, u_{d}(s, y), u_{1}(t, x), \ldots, u_{d}(t, x)\right)
$$

(the existence of $p_{t, x}(\cdot)$ is essentially a consequence of the result of BALLY AND PARDOUX [BP98], see our Corollary 4.3; the existence of $p_{s, y ; t, x}(\cdot, \cdot)$ is a consequence of Theorems 3.1 and 6.3).

Consider the following two hypotheses on the coefficients of the system (1.1):

$\mathbf{P 1}$ The functions $\sigma_{i j}$ and $b_{i}$ are bounded and infinitely differentiable with bounded partial derivatives of all orders, for $1 \leq i, j \leq d$.

P2 The matrix $\sigma$ is uniformly elliptic, that is, $\|\sigma(x) \xi\|^{2} \geq \rho^{2}>0$ for some $\rho>0$, for all $x \in \mathbb{R}^{d}, \xi \in \mathbb{R}^{d},\|\xi\|=1\left(\|\cdot\|\right.$ denotes the Euclidean norm on $\mathbb{R}^{d}$ ).

Theorem 1.1. Assume $\mathbf{P 1}$ and P2. Fix $T>0$ and let $I \subset(0, T]$ and $J \subset(0,1)$ be two compact nonrandom intervals.

(a) The density $p_{t, x}(z)$ is uniformly bounded over $z \in \mathbb{R}^{d}, t \in I$ and $x \in J$.

(b) There exists $c>0$ such that for any $t \in I, x \in J$ and $z \in \mathbb{R}^{d}$,

$$
p_{t, x}(z) \geq c t^{-d / 4} \exp \left(-\frac{\|z\|^{2}}{c t^{1 / 2}}\right) .
$$

(c) For all $\eta>0$, there exists $c>0$ such that for any $s, t \in I, x, y \in J,(s, y) \neq(t, x)$ and $z_{1}, z_{2} \in \mathbb{R}^{d}$

$$
p_{s, y ; t, x}\left(z_{1}, z_{2}\right) \leq c\left(|t-s|^{1 / 2}+|x-y|\right)^{-(d+\eta) / 2} \exp \left(-\frac{\left\|z_{1}-z_{2}\right\|^{2}}{c\left(|t-s|^{1 / 2}+|x-y|\right)}\right) .
$$


(d) There exists $c>0$ such that for any $t \in I, x, y \in J, x \neq y$ and $z_{1}, z_{2} \in \mathbb{R}^{d}$,

$$
p_{t, y ; t, x}\left(z_{1}, z_{2}\right) \leq c(|x-y|)^{-d / 2} \exp \left(-\frac{\left\|z_{1}-z_{2}\right\|^{2}}{c|x-y|}\right) .
$$

The main technical effort in this paper is to obtain the upper bound in (c). Indeed, it is not difficult to check that for fixed $(s, y ; t, x),\left(z_{1}, z_{2}\right) \mapsto p_{s, y ; t, x}\left(z_{1}, z_{2}\right)$ behaves like a Gaussian density function. However, for $(s, y)=(t, x)$, the $\mathbb{R}^{2 d}$-valued random vector $(u(s, y), u(t, x))$ is concentrated on a $d$-dimensional subspace in $\mathbb{R}^{2 d}$ and therefore does not have a density with respect to Lebesgue measure in $\mathbb{R}^{2 d}$. So the main effort is to estimate how this density blows up as $(s, y) \rightarrow(t, x)$. This is achieved by a detailed analysis of the behavior of the Malliavin matrix of $(u(s, y), u(t, x))$ as a function of $(s, y ; t, x)$, using a perturbation argument. The presence of $\eta$ in statement (c) may be due to the method of proof. When $t=s$, it is possible to set $\eta=0$ as in Theorem 1.1(d).

This paper is organized as follows. After introducing some notation and stating our main results on hitting probabilities (Theorems 1.2 and 1.6), we assume Theorem 1.1 and use the theorems of [DKN07] to prove these results in Section 2. In Section 3, we recall some basic facts of Malliavin calculus and state and prove two results that are tailored to our needs (Propositions 3.4 and 3.5). In Section 4, we establish the existence, smoothness and uniform boundedness of the one-point density function $p_{t, x}$, proving Theorem 1.1(a). In Section 5, we establish a lower bound on $p_{t, x}$, which proves Theorem 1.1(b). This upper (respectively lower) bound is a fairly direct extension to $d \geq 1$ of a result of BALLY AND PARDOUX [BP98] (respectively Kohatsu-Higa [K03]) when $d=1$. In Section 6, we establish Theorem 1.1(c) and $(\mathrm{d})$. The main steps are as follows.

The upper bound on the two-point density function $p_{s, y ; t, x}$ involves a bloc-decomposition of the Malliavin matrix of the $\mathbb{R}^{2 d}$-valued random vector $(u(s, y), u(s, y)-u(t, x))$. The entries of this matrix are of different orders of magnitude, depending on which bloc they are in: see Theorem 6.3. Assuming Theorem 6.3, we prove Theorem 1.1(c) and (d) in Section 6.3. The exponential factor in (1.5) is obtained from an exponential martingale inequality, while the factor $\left(|t-s|^{1 / 2}+|x-y|\right)^{-(d+\eta) / 2}$ comes from an estimate of the iterated Skorohod integrals that appear in Corollary 3.3 and from the block structure of the Malliavin matrix.

The proof of Theorem 6.3 is presented in Section 6.4: this is the main technical effort in this paper. We need bounds on the inverse of the Malliavin matrix. Bounds on its cofactors are given in Proposition 6.5, while bounds on negative moments of its determinant are given in Proposition 6.6. The determinant is equal to the product of the $2 d$ eigenvalues of the Malliavin matrix. It turns out that at least $d$ of these eigenvalues are of order 1 ("large eigenvalues") and do not contribute to the upper bound in (1.5), and at most $d$ are of the same order as the smallest eigenvalue ("small eigenvalues"), that is, of order $|t-s|^{1 / 2}+|x-y|$. If we did not distinguish between these two types of eigenvalues, but estimated all of them by the smallest eigenvalue, we would obtain a factor of $\left(|t-s|^{1 / 2}+|x-y|\right)^{-d+\eta / 2}$ in (1.5), which would not be the correct order. The estimates on the smallest eigenvalue are obtained by refining a technique that appears in [BP98]; indeed, we obtain a precise estimate on the density whereas they only showed existence. The study of the large eigenvalues does not seem to appear elsewhere in the literature. 
Coming back to potential theory, let us introduce some notation. For all Borel sets $F \subset \mathbb{R}^{d}$, we define $\mathscr{P}(F)$ to be the set of all probability measures with compact support contained in $F$. For all integers $k \geq 1$ and $\mu \in \mathscr{P}\left(\mathbb{R}^{k}\right)$, we let $I_{\beta}(\mu)$ denote the $\beta$-dimensional energy of $\mu$, that is,

$$
I_{\beta}(\mu):=\iint \mathrm{K}_{\beta}(\|x-y\|) \mu(d x) \mu(d y),
$$

where $\|x\|$ denotes the Euclidian norm of $x \in \mathbb{R}^{k}$,

$$
\mathrm{K}_{\beta}(r):= \begin{cases}r^{-\beta} & \text { if } \beta>0 \\ \log \left(N_{0} / r\right) & \text { if } \beta=0 \\ 1 & \text { if } \beta<0\end{cases}
$$

and $N_{0}$ is a sufficiently large constant (see Dalang, Khoshnevisan, And Nualart [DKN07, (1.5)].

For all $\beta \in \mathbb{R}$, integers $k \geq 1$, and Borel sets $F \subset \mathbb{R}^{k}, \operatorname{Cap}_{\beta}(F)$ denotes the $\beta$-dimensional capacity of $F$, that is,

$$
\operatorname{Cap}_{\beta}(F):=\left[\inf _{\mu \in \mathscr{P}(F)} I_{\beta}(\mu)\right]^{-1},
$$

where $1 / \infty:=0$. Note that if $\beta<0$, then $\operatorname{Cap}_{\beta}(\cdot) \equiv 1$.

Given $\beta \geq 0$, the $\beta$-dimensional Hausdorff measure of $F$ is defined by

$$
\mathscr{H}_{\beta}(F)=\lim _{\epsilon \rightarrow 0^{+}} \inf \left\{\sum_{i=1}^{\infty}\left(2 r_{i}\right)^{\beta}: F \subseteq \bigcup_{i=1}^{\infty} B\left(x_{i}, r_{i}\right), \sup _{i \geq 1} r_{i} \leq \epsilon\right\},
$$

where $B(x, r)$ denotes the open (Euclidean) ball of radius $r>0$ centered at $x \in \mathbb{R}^{d}$. When $\beta<0$, we define $\mathscr{H}_{\beta}(F)$ to be infinite. $[0,1]$,

Throughout, we consider the following parabolic metric: For all $s, t \in[0, T]$ and $x, y \in$

$$
\boldsymbol{\Delta}((t, x) ;(s, y)):=|t-s|^{1 / 2}+|x-y| .
$$

Clearly, this is a metric on $\mathbb{R}^{2}$ which generates the usual Euclidean topology on $\mathbb{R}^{2}$. Then we obtain an energy form

$$
I_{\beta}^{\Delta}(\mu):=\iint \mathrm{K}_{\beta}(\Delta((t, x) ;(s, y))) \mu(d t d x) \mu(d s d y)
$$

and a corresponding capacity

$$
\operatorname{Cap}_{\beta}^{\Delta}(F):=\left[\inf _{\mu \in \mathscr{P}(F)} I_{\beta}^{\Delta}(\mu)\right]^{-1} .
$$

For the Hausdorff measure, we write

$$
\mathscr{H}_{\beta}^{\boldsymbol{\Delta}}(F)=\lim _{\epsilon \rightarrow 0^{+}} \inf \left\{\sum_{i=1}^{\infty}\left(2 r_{i}\right)^{\beta}: F \subseteq \bigcup_{i=1}^{\infty} B^{\boldsymbol{\Delta}}\left(\left(t_{i}, x_{i}\right), r_{i}\right), \sup _{i \geq 1} r_{i} \leq \epsilon\right\},
$$


where $B^{\boldsymbol{\Delta}}((t, x), r)$ denotes the open $\boldsymbol{\Delta}$-ball of radius $r>0$ centered at $(t, x) \in[0, T] \times$ $[0,1]$.

Using Theorem 1.1 together with results from Dalang, KHOSHnEvisan, AND NualarT [DKN07], we shall prove the following result. Let $u(E)$ denote the (random) range of $E$ under the map $(t, x) \mapsto u(t, x)$, where $E$ is some Borel-measurable subset of $\mathbb{R}^{2}$.

Theorem 1.2. Assume $\mathbf{P 1}$ and P2. Fix $T>0, M>0$, and $\eta>0$. Let $I \subset(0, T]$ and $J \subset(0,1)$ be two fixed non-trivial compact intervals.

(a) There exists $c>0$ depending on $M, I, J$ and $\eta$ such that for all compact sets $A \subseteq$ $[-M, M]^{d}$,

$$
c^{-1} \operatorname{Cap}_{d-6+\eta}(A) \leq \mathrm{P}\{u(I \times J) \cap A \neq \emptyset\} \leq c \mathscr{H}_{d-6-\eta}(A) .
$$

(b) For all $t \in(0, T]$, there exists $c_{1}>0$ depending on $T, M$ and $J$, and $c_{2}>0$ depending on $T, M, J$ and $\eta>0$ such that for all compact sets $A \subseteq[-M, M]^{d}$,

$$
c_{1} \operatorname{Cap}_{d-2}(A) \leq \mathrm{P}\{u(\{t\} \times J) \cap A \neq \emptyset\} \leq c_{2} \mathscr{H}_{d-2-\eta}(A) .
$$

(c) For all $x \in(0,1)$, there exists $c>0$ depending on $M, I$ and $\eta$ such that for all compact sets $A \subseteq[-M, M]^{d}$,

$$
c^{-1} \operatorname{Cap}_{d-4+\eta}(A) \leq \mathrm{P}\{u(I \times\{x\}) \cap A \neq \emptyset\} \leq c \mathscr{H}_{d-4-\eta}(A) .
$$

Remark 1.3. (i) Because of the inequalities between capacity and Hausdorff measure, the right-hand sides of Theorem 1.2 can be replaced by $c \operatorname{Cap}_{d-6-\eta}(A), c \operatorname{Cap}_{d-2-\eta}(A)$ and $c \operatorname{Cap}_{d-4-\eta}(A)$ in (a), (b) and (c), respectively (cf. KaHANe [K85, p. 133]).

(ii) Theorem 1.2 also holds if we consider Dirichlet boundary conditions (i.e. $u_{i}(t, 0)=$ $u_{i}(t, 1)=0$, for $\left.t \in[0, T]\right)$ instead of Neumann boundary conditions.

(iii) In the upper bounds of Theorem 1.2, the condition in $\mathbf{P} \mathbf{1}$ that $\sigma$ and $b$ are bounded can be removed, but their derivatives of all orders must exist and be bounded.

As a consequence of Theorem 1.2, we deduce the following result on the polarity of points. Recall that a Borel set $A \subseteq \mathbb{R}^{d}$ is called polar for $u$ if $\mathrm{P}\{u((0, T] \times(0,1)) \cap A \neq$ $\varnothing\}=0$; otherwise, $A$ is called nonpolar.

Corollary 1.4. Assume $\mathbf{P 1}$ and $\mathbf{P 2}$.

(a) Singletons are nonpolar for $(t, x) \mapsto u(t, x)$ when $d \leq 5$, and are polar when $d \geq 7$ (the case $d=6$ is open).

(b) Fix $t \in(0, T]$. Singletons are nonpolar for $x \mapsto u(t, x)$ when $d=1$, and are polar when $d \geq 3$ (the case $d=2$ is open).

(c) Fix $x \in(0,1)$. Singletons are not polar for $t \mapsto u(t, x)$ when $d \leq 3$ and are polar when $d \geq 5$ (the case $d=4$ is open). 
Another consequence of Theorem 1.2 is the Hausdorff dimension of the range of the process $u$.

Corollary 1.5. Assume $\mathbf{P} 1$ and $\mathbf{P 2}$.

(a) If $d>6$, then $\operatorname{dim}_{\mathrm{H}}(u((0, T] \times(0,1)))=6$ a.s.

(b) Fix $t \in \mathbb{R}_{+}$. If $d>2$, then $\operatorname{dim}_{\mathrm{H}}(u(\{t\} \times(0,1)))=2$ a.s.

(c) Fix $x \in(0,1)$. If $d>4$, then $\operatorname{dim}_{\mathrm{H}}\left(u\left(\mathbb{R}_{+} \times\{x\}\right)\right)=4$ a.s.

As in Dalang, Khoshnevisan, and Nualart [DKN07], it is also possible to use Theorem 1.1 to obtain results concerning level sets of $u$. Define

$$
\begin{aligned}
& \mathscr{L}(z ; u):=\{(t, x) \in I \times J: u(t, x)=z\}, \\
& \mathscr{T}(z ; u)=\{t \in I: u(t, x)=z \text { for some } x \in J\}, \\
& \mathscr{X}(z ; u)=\{x \in J: u(t, x)=z \text { for some } t \in I\}, \\
& \mathscr{L}_{x}(z ; u):=\{t \in I: u(t, x)=z\}, \\
& \mathscr{L}^{t}(z ; u):=\{x \in J: u(t, x)=z\} .
\end{aligned}
$$

We note that $\mathscr{L}(z ; u)$ is the level set of $u$ at level $z, \mathscr{T}(z ; u)$ (resp. $\mathscr{X}(z ; u))$ is the projection of $\mathscr{L}(z ; u)$ onto $I$ (resp. $J)$, and $\mathscr{L}_{x}(z ; u)$ (resp. $\left.\mathscr{L}^{t}(z ; u)\right)$ is the $x$-section (resp. $t$-section) of $\mathscr{L}(z ; u)$.

Theorem 1.6. Assume $\mathbf{P} 1$ and $\mathbf{P 2}$. Then for all $\eta>0$ and $R>0$ there exists a positive and finite constant $c$ such that the following holds for all compact sets $E \subset(0, T] \times(0,1)$, $F \subset(0, T], G \subset(0,1)$, and for all $z \in B(0, R)$ :

(a) $c^{-1} \operatorname{Cap}_{(d+\eta) / 2}^{\Delta}(E) \leq \mathrm{P}\{\mathscr{L}(z ; u) \cap E \neq \varnothing\} \leq c \mathscr{H}_{(d-\eta) / 2}^{\Delta}(E)$;

(b) $c^{-1} \operatorname{Cap}_{(d-2+\eta) / 4}(F) \leq \mathrm{P}\{\mathscr{T}(z ; u) \cap F \neq \varnothing\} \leq c \mathscr{H}_{(d-2-\eta) / 4}(F)$;

(c) $c^{-1} \operatorname{Cap}_{(d-4+\eta) / 2}(G) \leq \mathrm{P}\{\mathscr{X}(z ; u) \cap G \neq \varnothing\} \leq c \mathscr{H}_{(d-4-\eta) / 2}(G)$;

(d) for all $x \in(0,1), c^{-1} \operatorname{Cap}_{(d+\eta) / 4}(F) \leq \mathrm{P}\left\{\mathscr{L}_{x}(z ; u) \cap F \neq \varnothing\right\} \leq c \mathscr{H}_{(d-\eta) / 4}(F)$;

(e) for all $t \in(0, T], c^{-1} \operatorname{Cap}_{d / 2}(G) \leq \mathrm{P}\left\{\mathscr{L}^{t}(z ; u) \cap G \neq \varnothing\right\} \leq c \mathscr{H}_{(d-\eta) / 2}(G)$.

Corollary 1.7. Assume $\mathbf{P 1}$ and $\mathbf{P 2}$. Choose and fix $z \in \mathbb{R}^{d}$.

(a) If $2<d<6$, then $\operatorname{dim}_{\mathrm{H}} \mathscr{T}(z ; u)=\frac{1}{4}(6-d)$ a.s. on $\{\mathscr{T}(z ; u) \neq \varnothing\}$.

(b) If $4<d<6$ (i.e. $d=5$ ), then $\operatorname{dim}_{\mathrm{H}} \mathscr{X}(z ; u)=\frac{1}{2}(6-d)$ a.s. on $\{\mathscr{X}(z ; u) \neq \varnothing\}$.

(c) If $1 \leq d<4$, then $\operatorname{dim}_{\mathrm{H}} \mathscr{L}_{x}(z ; u)=\frac{1}{4}(4-d)$ a.s. on $\left\{\mathscr{L}_{x}(z ; u) \neq \varnothing\right\}$.

(d) If $d=1$, then $\operatorname{dim}_{\mathrm{H}} \mathscr{L}^{t}(z ; u)=\frac{1}{2}(2-d)=\frac{1}{2}$ a.s. on $\left\{\mathscr{L}^{t}(z ; u) \neq \varnothing\right\}$.

In addition, all four right-most events have positive probability.

Remark 1.8. The results of the two theorems and corollaries above should be compared with those of Dalang, Khsohnevisan and Nualart [DKN07]. 


\section{Proof of Theorems 1.2, 1.6 and their corollaries (assuming Theorem 1.1)}

We start this section recalling the hypotheses and consequences of Theorems 3.3 and 2.1 in Dalang, Khsohnevisan and Nualart [DKN07].

Theorem 2.1. [DKN07, Theorem 3.3] Consider two compact nonrandom intervals $I \subset$ $[0, T]$ and $J \subset[0,1]$, and suppose $v=\{v(t, x)\}_{(t, x) \in I \times J}$ is an $\mathbb{R}^{d}$-valued random field. For $M>0$ fixed, assume the following two conditions:

(i) For any $(t, x) \in I \times J$, the random vector $v(t, x)$ has a density $p_{t, x}(z)$ which is is uniformly bounded over $z \in[-M, M]^{d}$ and $(t, x) \in I \times J$.

(ii) For all $p>1$, there exists a constant $C$ depending on $p, I, J$ such that for any $(t, x),(s, y) \in I \times J$,

$$
\mathrm{E}\left[|v(t, x)-v(s, y)|^{p}\right] \leq C[\boldsymbol{\Delta}((t, x) ;(s, y))]^{p / 2} .
$$

Then for any $\beta \in] 0, d[$, there exists a positive and finite constant a such that for all Borel sets $A \subset[-M, M]^{d}$ :

(1) $\mathrm{P}\{v(I \times J) \cap A \neq \varnothing\} \leq a \mathscr{H}_{\beta-6}(A)$;

(2) for every $t \in I, \mathrm{P}\{v(\{t\} \times J) \cap A \neq \varnothing\} \leq a \mathscr{H}_{\beta-2}(A)$;

(3) for every $x \in J, \mathrm{P}\{v(I \times\{x\}) \cap A \neq \varnothing\} \leq a \mathscr{H}_{\beta-4}(A)$.

Theorem 2.2. [DKN07, Theorem 2.1] Fix two compact intervals $I$ and $J$ of $\mathbb{R}$. Suppose that $\{v(t, x)\}_{(t, x) \in I \times J}$ is a two-parameter, continuous random field with values in $\mathbb{R}^{d}$, such that $(v(t, x), v(s, y))$ has a joint probability density function $p_{t, x ; s, y}(\cdot, \cdot)$, for all $s, t \in I$ and $x, y \in J$ such that $(t, x) \neq(s, y)$. We denote by $p_{t, x}(\cdot)$ the density function of $v(t, x)$. Assume the following hypotheses:

A1. For all $M>0$, there exists a positive and finite constant $C=C(I, J, M, d)$ such that for all $(t, x) \in I \times J$ and all $z \in[-M, M]^{d}$,

$$
p_{t, x}(z) \geq C \text {. }
$$

A2. There exists $\beta>0$ such that for all $M>0$, there exists $c=c(I, J, \beta, M, d)>0$ such that for all $s, t \in I$ and $x, y \in J$ with $(t, x) \neq(s, y)$, and for every $z_{1}, z_{2} \in[-M, M]^{d}$,

$$
p_{t, x ; s, y}\left(z_{1}, z_{2}\right) \leq \frac{c}{[\boldsymbol{\Delta}((t, x) ;(s, y))]^{\beta / 2}} \exp \left(-\frac{\left\|z_{1}-z_{2}\right\|^{2}}{c \boldsymbol{\Delta}((t, x) ;(s, y))}\right) .
$$

Then the following inequalities hold.

(1) There exists a positive and finite constant $a=a(I, J, \beta, M, d)$ such that for all compact sets $A \subseteq[-M, M]^{d}$,

$$
\mathrm{P}\{v(I \times J) \cap A \neq \varnothing\} \geq a \operatorname{Cap}_{\beta-6}(A) .
$$


(2) There exists a positive and finite constant $a=a(J, M \beta, d)$ such that for all $t \in I$ and for all compact sets $A \subseteq[-M, M]^{d}$,

$$
\mathrm{P}\{v(\{t\} \times J) \cap A \neq \varnothing\} \geq a \operatorname{Cap}_{\beta-2}(A) .
$$

(3) There exists a positive and finite constant $a=a(I, M, \beta, d)$ such that for all $x \in J$ and for all compact sets $A \subseteq[-M, M]^{d}$,

$$
\mathrm{P}\{v(I \times\{x\}) \cap A \neq \varnothing\} \geq a \operatorname{Cap}_{\beta-4}(A) .
$$

We shall apply these two theorems to the solution of equation (1.1). We first recall that equation (1.1) is formal: a rigorous formulation, following WALSH [W86], is as follows. Let $W^{i}=\left(W^{i}(s, x)\right)_{s \in \mathbb{R}_{+}, x \in[0,1]}, i=1, \ldots, d$, be independent Brownian sheets defined on a probability space $(\Omega, \mathscr{F}, \mathrm{P})$, and set $W=\left(W^{1}, \ldots, W^{d}\right)$. For $t \geq 0$, let $\mathscr{F}_{t}=\sigma\{W(s, x), s \in$ $[0, t], x \in[0,1]\}$. We say that a process $u=\{u(t, x), t \in[0, T], x \in[0,1]\}$ is adapted to $\left(\mathscr{F}_{t}\right)$ if $u(t, x)$ is $\mathscr{F}_{t}$-measurable for each $(t, x) \in[0, T] \times[0,1]$. We say that $u$ is a solution of (1.1) if $u$ is adapted to $\left(\mathscr{F}_{t}\right)$ and if for $i \in\{1, \ldots, d\}$,

$$
\begin{aligned}
u_{i}(t, x)=\int_{0}^{t} \int_{0}^{1} & G_{t-r}(x, v) \sum_{j=1}^{d} \sigma_{i, j}(u(r, v)) W^{j}(d r, d v) \\
& +\int_{0}^{t} \int_{0}^{1} G_{t-r}(x, v) b_{i}(u(r, v)) d r d v
\end{aligned}
$$

where $G_{t}(x, y)$ denotes the Green kernel for the heat equation with Neumann boundary conditions (see WALSH [W86, Chap 3]), and the stochastic integral in (2.2) is interpreted as in [W86].

Adapting the results from [W86] to the case $d \geq 1$, one can show that there exists a unique continuous process $u=\{u(t, x), t \in[0, T], x \in[0,1]\}$ adapted to $\left(\mathscr{F}_{t}\right)$ that is a solution of (1.1). Moreover, it is shown in Bally, Millet, AND Sanz-Solé [BMS95] that for any $s, t \in[0, T]$ with $s \leq t, x, y \in[0,1]$, and $p>1$,

$$
\mathrm{E}\left[|u(t, x)-u(s, y)|^{p}\right] \leq C_{T, p}(\boldsymbol{\Delta}((t, x) ;(s, y)))^{p / 2},
$$

where $\boldsymbol{\Delta}$ is the parabolic metric defined in (1.8). In particular, for any $0<\alpha<1 / 2, u$ is a.s. $\alpha$-Hölder continuous in $x$ and $\alpha / 2$-Hölder continuous in $t$.

Assuming Theorem 1.1, we now prove Theorems 1.2, 1.6 and their corollaries.

Proof of Theorem 1.2. (a) In order to prove the upper bound, we use Theorem 2.1. Indeed, Theorem 1.1(a) and (2.3) imply that the hypotheses (i) and (ii), respectively, of this theorem, are satisfied, and so the conclusion (with $\beta=d-\eta$ ) is too.

In order to prove the lower bound, we shall use Theorem 2.2. Hypothesis A1 is an immediate consequence of Theorem 1.1(b). Hypothesis A2 holds with $\beta=d+\eta$ by Theorem 1.1(c). Therefore, the lower bound in Theorem 1.2(a) follows from Theorem 2.2(1). This proves (a).

(b) For the upper bound, we again refer to Theorem 2.1. For the lower bound, which involves $\operatorname{Cap}_{d-2}(A)$ instead of $\operatorname{Cap}_{d-2+\eta}(A)$, we refer to [DKN07, Remark 2.5] and observe 
that hypotheses $\mathbf{A} 1^{t}$ and $\mathbf{A} \mathbf{2}^{t}$ there are satisfied with $\beta=d$ (by Theorem 1.1(d)). This proves (b).

(c) As in (a), the upper bound follows from Theorem 2.1 with $\beta=d-\eta$, and the lower bound follows from Theorem 2.2(3), with $\beta=d+\eta$. Theorem 1.2 is proved.

Proof of Corollary 1.4. We first prove (a). Let $z \in \mathbb{R}^{d}$. If $d \leq 5$, then there is $\eta>0$ such that $d-6+\eta<0$, and thus $\operatorname{Cap}_{d-6+\eta}(\{z\})=1$. Hence, the lower bound of Theorem 1.2 (a) implies that $\{z\}$ is not polar. On the other hand, if $d>6$, then for small $\eta>0$, $d-6-\eta>0$. Therefore, $\mathscr{H}_{d-6-\eta}(\{z\})=0$ and the upper bound of Theorem 1.2(a) implies that $\{z\}$ is polar. This proves (a). One proves (b) and (c) exactly along the same lines using Theorem 1.2(b) and (c).

Proof of Theorem 1.6. For the upper bounds in (a)-(e), we use Dalang, Khoshnevisan, AND NUALART [DKN07, Theorems 3.3 and 3.1] whose assumptions we verified above with $\beta=d-\eta$; these upper bounds then follow immediately from [DKN07, Theorem 3.2].

For the lower bounds in (a)-(d), we use [DKN07, Theorem 2.4] since we have shown above that the assumptions of this theorem, with $\beta=d+\eta$, are satisfied by Theorem 1.1. For the lower bound in (e), we refer to [DKN07, Remark 2.5] and note that by Theorem 1.1(d), Hypothesis $\mathbf{A} 2^{t}$ there is satisfied with $\beta=d$. This proves Theorem 1.6.

Proof of Corollaries 1.5 and 1.7. The final positive-probability assertion in Corollary 1.7 is an immediate consequence of Theorem 1.6 and Taylor's theorem (see KHOSHNEVISAN [K02, Corollary 2.3.1 p. 523]).

Let $E$ be a random set. When it exists, the codimension of $E$ is the real number $\beta \in[0, d]$ such that for all compact sets $A \subset \mathbb{R}^{d}$,

$$
\mathrm{P}\{E \cap A \neq \varnothing\} \begin{cases}>0 & \text { whenever } \operatorname{dim}_{\mathrm{H}}(A)>\beta, \\ =0 & \text { whenever } \operatorname{dim}_{\mathrm{H}}(A)<\beta .\end{cases}
$$

See Khoshnevisan [K02, Chap.11, Section 4]. When it is well defined, we write the said codimension as $\operatorname{codim}(E)$. Theorems 1.2 and 1.6 imply that for $d \geq 1: \operatorname{codim}\left(u\left(\mathbb{R}_{+} \times\right.\right.$ $(0,1)))=(d-6)^{+} ; \operatorname{codim}(u(\{t\} \times(0,1)))=(d-2)^{+} ; \operatorname{codim}\left(u\left(\mathbb{R}_{+} \times\{x\}\right)\right)=(d-4)^{+} ;$ $\operatorname{codim}(\mathscr{T}(z))=\left(\frac{d-2}{4}\right)^{+} ; \operatorname{codim}(\mathscr{X}(z))=\left(\frac{d-4}{2}\right)^{+} ; \operatorname{codim}\left(\mathscr{L}_{x}(z)\right)=\frac{d}{4} ;$ and $\operatorname{codim}\left(\mathscr{L}^{t}(z)\right)=$ $\frac{d}{2}$. According to Theorem 4.7.1 of Khoshnevisan [K02, Chapter 11], given a random set $E$ in $\mathbb{R}^{n}$ whose codimension is strictly between 0 and $n$,

$$
\operatorname{dim}_{\mathrm{H}} E+\operatorname{codim} E=n \quad \text { a.s. on }\{E \neq \varnothing\} .
$$

This implies the statements of Corollaries 1.5 and 1.7.

\section{Elements of Malliavin calculus}

In this section, we introduce, following NualarT [N95] (see also SANZ-Solé [S05]), some elements of Malliavin calculus. Let $\mathscr{S}$ denote the class of smooth random variables of the form

$$
F=f\left(W\left(h_{1}\right), \ldots, W\left(h_{n}\right)\right),
$$


where $n \geq 1, f \in \mathscr{C}_{P}^{\infty}\left(\mathbb{R}^{n}\right)$, the set of real-valued functions $f$ such that $f$ and all its partial derivatives have at most polynomial growth, $h_{i} \in \mathscr{H}:=L^{2}\left([0, T] \times[0,1], \mathbb{R}^{d}\right)$, and $W\left(h_{i}\right)$ denotes the Wiener integral

$$
W\left(h_{i}\right)=\int_{0}^{T} \int_{0}^{1} h_{i}(t, x) \cdot W(d x, d t), \quad 1 \leq i \leq n .
$$

Given $F \in \mathscr{S}$, its derivative is defined to be the $\mathbb{R}^{d}$-valued stochastic process $D F=$ $\left(D_{t, x} F=\left(D_{t, x}^{(1)} F, \ldots, D_{t, x}^{(d)} F\right),(t, x) \in[0, T] \times[0,1]\right)$ given by

$$
D_{t, x} F=\sum_{i=1}^{n} \frac{\partial f}{\partial x_{i}}\left(W\left(h_{1}\right), \ldots, W\left(h_{n}\right)\right) h_{i}(t, x) .
$$

More generally, we can define the derivative $D^{k} F$ of order $k$ of $F$ by setting

$$
D_{\alpha}^{k} F=\sum_{i_{1}, \ldots, i_{k}=1}^{n} \frac{\partial}{\partial x_{i_{1}}} \cdots \frac{\partial}{\partial x_{i_{k}}} f\left(W\left(h_{1}\right), \ldots, W\left(h_{n}\right)\right) h_{i_{1}}\left(\alpha_{1}\right) \otimes \cdots \otimes h_{i_{k}}\left(\alpha_{k}\right),
$$

where $\alpha=\left(\alpha_{1}, \ldots, \alpha_{k}\right)$, and $\alpha_{i}=\left(t_{i}, x_{i}\right), 1 \leq i \leq k$.

For $p, k \geq 1$, the space $\mathbb{D}^{k, p}$ is the closure of $\mathscr{S}$ with respect to the seminorm $\|\cdot\|_{k, p}^{p}$ defined by

$$
\|F\|_{k, p}^{p}=\mathrm{E}\left[|F|^{p}\right]+\sum_{j=1}^{k} \mathrm{E}\left[\left\|D^{j} F\right\|_{\mathscr{H}^{\otimes j}}^{p}\right]
$$

where

$$
\left\|D^{j} F\right\|_{\mathscr{H}^{\otimes j}}^{2}=\sum_{i_{1}, \ldots, i_{j}=1}^{d} \int_{0}^{T} d t_{1} \int_{0}^{1} d x_{1} \cdots \int_{0}^{T} d t_{j} \int_{0}^{1} d x_{j}\left(D_{\left(t_{1}, x_{1}\right)}^{\left(i_{1}\right)} \cdots D_{\left(t_{j}, x_{j}\right)}^{\left(i_{j}\right)} F\right)^{2} .
$$

We set $\left(\mathbb{D}^{\infty}\right)^{d}=\cap_{p \geq 1} \cap_{k \geq 1} \mathbb{D}^{k, p}$.

The derivative operator $D$ on $L^{2}(\Omega)$ has an adjoint, termed the Skorohod integral and denoted by $\delta$, which is an unbounded operator on $L^{2}(\Omega, \mathscr{H})$. Its domain, denoted by Dom $\delta$, is the set of elements $u \in L^{2}(\Omega, \mathscr{H})$ such that there exists a constant $c$ such that $\left|E\left[\langle D F, u\rangle_{\mathscr{H}}\right]\right| \leq c\|F\|_{0,2}$, for any $F \in \mathbb{D}^{1,2}$.

If $u \in \operatorname{Dom} \delta$, then $\delta(u)$ is the element of $L^{2}(\Omega)$ characterized by the following duality relation:

$$
\mathrm{E}[F \delta(u)]=\mathrm{E}\left[\sum_{j=1}^{d} \int_{0}^{T} \int_{0}^{1} D_{t, x}^{(j)} F u_{j}(t, x) d t d x\right] \text {, for all } F \in \mathbb{D}^{1,2} .
$$

A first application of Malliavin calculus to the study of probability laws is the following global criterion for smoothness of densities.

Theorem 3.1. [N95, Thm.2.1.2 and Cor.2.1.2] or [S05, Thm.5.2] Let $F=\left(F^{1}, \ldots, F^{d}\right)$ be an $\mathbb{R}^{d}$-valued random vector satisfying the following two conditions: 
(i) $F \in\left(\mathbb{D}^{\infty}\right)^{d}$;

(ii) the Malliavin matrix of $F$ defined by $\gamma_{F}=\left(\left\langle D F^{i}, D F^{j}\right\rangle_{\mathscr{H}}\right)_{1 \leq i, j \leq d}$ is invertible a.s. and $\left(\operatorname{det} \gamma_{F}\right)^{-1} \in L^{p}(\Omega)$ for all $p \geq 1$.

Then the probability law of $F$ has an infinitely differentiable density function.

A random vector $F$ that satisfies conditions (i) and (ii) of Theorem 3.1 is said to be nondegenerate. For a nondegenerate random vector, the following integration by parts formula plays a key role.

Proposition 3.2. [N98, Prop.3.2.1] or [S05, Prop.5.4] Let $F=\left(F^{1}, \ldots, F^{d}\right) \in\left(\mathbb{D}^{\infty}\right)^{d}$ be a nondegenerate random vector, let $G \in \mathbb{D}^{\infty}$ and let $g \in \mathscr{C}_{P}^{\infty}\left(\mathbb{R}^{d}\right)$. Fix $k \geq 1$. Then for any multi-index $\alpha=\left(\alpha_{1}, \ldots, \alpha_{k}\right) \in\{1, \ldots, d\}^{k}$, there is an element $H_{\alpha}(F, G) \in \mathbb{D}^{\infty}$ such that

$$
\mathrm{E}\left[\left(\partial_{\alpha} g\right)(F) G\right]=\mathrm{E}\left[g(F) H_{\alpha}(F, G)\right] .
$$

In fact, the random variables $H_{\alpha}(F, G)$ are recursively given by

$$
\begin{aligned}
H_{\alpha}(F, G) & =H_{\left(\alpha_{k}\right)}\left(F, H_{\left(\alpha_{1}, \ldots, \alpha_{k-1}\right)}(F, G)\right), \\
H_{(i)}(F, G) & =\sum_{j=1}^{d} \delta\left(G\left(\gamma_{F}^{-1}\right)_{i, j} D F^{j}\right) .
\end{aligned}
$$

Proposition 3.2 with $G=1$ and $\alpha=(1, \ldots, d)$ implies the following expression for the density of a nondegenerate random vector.

Corollary 3.3. [N98, Corollary 3.2.1] Let $F=\left(F^{1}, \ldots, F^{d}\right) \in\left(\mathbb{D}^{\infty}\right)^{d}$ be a nondegenerate random vector and let $p_{F}(z)$ denote the density of $F$. Then for every subset $\sigma$ of the set of indices $\{1, \ldots, d\}$,

$$
p_{F}(z)=(-1)^{d-|\sigma|} \mathrm{E}\left[1_{\left\{F^{i}>z^{i}, i \in \sigma, F^{i}<z^{i}, i \notin \sigma\right\}} H_{(1, \ldots, d)}(F, 1)\right],
$$

where $|\sigma|$ is the cardinality of $\sigma$, and, in agreement with Proposition 3.2,

$$
H_{(1, \ldots, d)}(F, 1)=\delta\left(\left(\gamma_{F}^{-1} D F\right)^{d} \delta\left(\left(\gamma_{F}^{-1} D F\right)^{d-1} \delta\left(\cdots \delta\left(\left(\gamma_{F}^{-1} D F\right)^{1}\right) \cdots\right)\right)\right) .
$$

The next result gives a criterion for uniform boundedness of the density of a nondegenerate random vector.

Proposition 3.4. For all $p>1$ and $\ell \geq 1$, let $c_{1}=c_{1}(p)>0$ and $c_{2}=c_{2}(\ell, p) \geq 0$ be fixed. Let $F \in\left(\mathbb{D}^{\infty}\right)^{d}$ be a nondegenerate random vector such that

(a) $\mathrm{E}\left[\left(\operatorname{det} \gamma_{F}\right)^{-p}\right] \leq c_{1}$;

(b) $\mathrm{E}\left[\left\|D^{l}\left(F^{i}\right)\right\|_{\mathscr{H} \otimes \ell}^{p}\right] \leq c_{2}, i=1, \ldots, d$.

Then the density of $F$ is uniformly bounded, and the bound does not depend on $F$ but only on the constants $c_{1}(p)$ and $c_{2}(\ell, p)$. 
Proof. The proof of this result uses the same arguments as in the proof of DALANG AND NualarT [DN04, Lemma 4.11]. Therefore, we will only give the main steps.

Fix $z \in \mathbb{R}^{d}$. Thanks to Corollary 3.3 and the Cauchy-Schwarz inequality we find that

$$
\left|p_{F}(z)\right| \leq\left\|H_{(1, \ldots, d)}(F, 1)\right\|_{0,2} .
$$

Using the continuity of the Skorohod integral $\delta$ (cf. Nualart [N95, Proposition 3.2.1] and NualarT [N98, (1.11) and p.131]) and Hölder's inequality for Malliavin norms (cf. Watanabe [W84, Proposition 1.10, p.50]), we obtain

$$
\left\|H_{(1, \ldots, d)}(F, 1)\right\|_{0,2} \leq c\left\|H_{(1, \ldots, d-1)}(F, 1)\right\|_{1,4} \sum_{j=1}^{d}\left\|\left(\gamma_{F}^{-1}\right)_{d, j}\right\|_{1,8}\left\|D\left(F^{j}\right)\right\|_{1,8} .
$$

In agreement with hypothesis (b), $\left\|D\left(F^{j}\right)\right\|_{m, p} \leq c$. In order to bound the second factor in (3.1), note that

$$
\left\|\left(\gamma_{F}^{-1}\right)_{i, j}\right\|_{m, p}=\left\{\mathrm{E}\left[\left|\left(\gamma_{F}^{-1}\right)_{i, j}\right|^{p}\right]+\sum_{k=1}^{m} \mathrm{E}\left[\left\|D^{k}\left(\gamma_{F}^{-1}\right)_{i, j}\right\|_{\mathscr{H} \otimes k}^{p}\right]\right\}^{1 / p} .
$$

For the first term in (3.2), we use Cramer's formula to get that

$$
\left|\left(\gamma_{F}^{-1}\right)_{i, j}\right|=\left|\left(\operatorname{det} \gamma_{F}\right)^{-1}\left(A_{F}\right)_{i, j}\right|,
$$

where $A_{F}$ denotes the cofactor matrix of $\gamma_{F}$. By means of Cauchy-Schwarz inequality and hypotheses (a) and (b) we find that

$$
\begin{aligned}
& \mathrm{E}\left[\left(\left(\gamma_{F}^{-1}\right)_{i, j}\right)^{p}\right] \leq c_{d, p}\left\{\mathrm{E}\left[\left(\operatorname{det} \gamma_{F}\right)^{-2 p}\right]\right\}^{1 / 2} \times\left\{\mathrm{E}\left[\|D(F)\|_{\mathscr{H}}^{4 p(d-1)}\right]\right\}^{1 / 2} \\
& \quad \leq c_{d, p}
\end{aligned}
$$

where none of the constants depend on $F$. For the second term on the right-hand side of (3.2), we iterate the equality (cf. NuAlarT [N95, Lemma 2.1.6])

$$
D\left(\gamma_{F}^{-1}\right)_{i, j}=-\sum_{k, \ell=1}^{d}\left(\gamma_{F}^{-1}\right)_{i, k} D\left(\gamma_{F}\right)_{k, \ell}\left(\gamma_{F}^{-1}\right)_{\ell, j}
$$

in the same way as in the proof of DALANG AND NuALART [DN04, Lemma 4.11]. Then, appealing again to hypotheses (a) and (b) and iterating the inequality (3.1) to bound the first factor on the right-hand side of (3.2), we obtain the uniform boundedness of $p_{F}(z)$.

We finish this section with a result that will be used later on to bound negative moments of a random variable, as is needed to check hypothesis (a) of Proposition 3.4.

Proposition 3.5. Suppose $Z \geq 0$ is a random variable for which we can find $\epsilon_{0} \in(0,1)$, processes $\left\{Y_{i, \epsilon}\right\}_{\epsilon \in(0,1)}(i=1,2)$, and constants $c>0$ and $0 \leq \alpha_{2} \leq \alpha_{1}$ with the property that 
$Z \geq \min \left(c \epsilon^{\alpha_{1}}-Y_{1, \epsilon} c \epsilon^{\alpha_{2}}-Y_{2, \epsilon}\right)$ for all $\epsilon \in\left(0, \epsilon_{0}\right)$. Also suppose that we can find $\beta_{i}>\alpha_{i}$ $(i=1,2)$, not depending on $\epsilon_{0}$, such that

$$
C(q):=\sup _{0<\epsilon<1} \max \left(\frac{\mathrm{E}\left[\left|Y_{1, \epsilon}\right|^{q}\right]}{\epsilon^{q \beta_{1}}}, \frac{\mathrm{E}\left[\left|Y_{2, \epsilon}\right|^{q}\right]}{\epsilon^{q \beta_{2}}}\right)<\infty \quad \text { for all } q \geq 1 .
$$

Then for all $p \geq 1$, there exists a constant $c^{\prime} \in(0, \infty)$, not depending on $\epsilon_{0}$, such that $\mathrm{E}\left[|Z|^{-p}\right] \leq c^{\prime} \epsilon_{0}^{-p \alpha_{1}}$.

Remark 3.6. This lemma is of interest mainly when $\beta_{2} \leq \alpha_{1}$.

Proof. Define $k:=(2 / c) \epsilon_{0}^{-\alpha_{1}}$. Suppose that $y \geq k$, and let $\epsilon:=(2 / c)^{1 / \alpha_{1}} y^{-1 / \alpha_{1}}$. Then $0<\epsilon \leq \epsilon_{0}, y^{-1}=(c / 2) \epsilon^{\alpha_{1}}$, and for all $q \geq 1$,

$$
\begin{aligned}
\mathrm{P}\left\{Z^{-1}>y\right\} & =\mathrm{P}\left\{Z<y^{-1}\right\} \\
& \leq \mathrm{P}\left\{Y_{1, \epsilon} \geq \frac{c}{2} \epsilon^{\alpha_{1}}\right\}+\mathrm{P}\left\{Y_{2, \epsilon} \geq c \epsilon^{\alpha_{2}}-\frac{c}{2} \epsilon^{\alpha_{1}}\right\} \\
& \leq \frac{C(q)}{c^{q}}\left(2^{q} \epsilon^{q\left(\beta_{1}-\alpha_{1}\right)}+\epsilon^{q \beta_{2}}\left[\epsilon^{\alpha_{2}}-\frac{1}{2} \epsilon^{\alpha_{1}}\right]^{-q}\right) .
\end{aligned}
$$

The inequality $\epsilon^{\alpha_{2}}-(1 / 2) \epsilon^{\alpha_{1}} \geq(1 / 2) \epsilon^{\alpha_{2}}$ implies that

$$
\mathrm{P}\left\{Z^{-1}>y\right\} \leq \frac{C(q)}{c^{q}}\left(2^{q} \epsilon^{q\left(\beta_{1}-\alpha_{1}\right)}+2^{q} \epsilon^{q\left(\beta_{2}-\alpha_{2}\right)}\right) \leq a y^{-q b},
$$

where $a$ and $b$ are positive and finite constants that do not depend on $y, \epsilon_{0}$ or $q$. We apply this with $q:=(p / b)+1$ to find that for all $p \geq 1$,

$$
\begin{aligned}
\mathrm{E}\left[|Z|^{-p}\right] & =p \int_{0}^{\infty} y^{p-1} \mathrm{P}\left\{Z^{-1}>y\right\} d y \\
& \leq k^{p}+a p \int_{k}^{\infty} y^{-b-1} d y=k^{p}+\left(\frac{a p}{b}\right) k^{-b} .
\end{aligned}
$$

Because $k \geq(2 / c)$ and $b>0$, it follows that $\mathrm{E}\left[|Z|^{-p}\right] \leq\left(1+c_{1}(a p / b)\right) k^{p}$, where $c_{1}:=$ $(c / 2)^{b+p}$. This is the desired result.

\section{Existence, smoothness and uniform boundedness of the one-point density}

Let $u=\{u(t, x), t \in[0, T], x \in[0,1]\}$ be the solution of equation (2.2). In this section, we prove the existence, smoothness and uniform boundedness of the density of the random vector $u(t, x)$. In particular, this will prove Theorem 1.1(a).

The first result concerns the Malliavin differentiability of $u$ and the equations satisfied by its derivatives. We refer to BALly AND PARDoux [BP98, Proposition 4.3, (4.16), (4.17)] for its proof in dimension one. As we work coordinate by coordinate, the following proposition follows in the same way and its proof is therefore omitted. 
Proposition 4.1. Assume P1. Then $u(t, x) \in\left(\mathbb{D}^{\infty}\right)^{d}$ for any $t \in[0, T]$ and $x \in[0,1]$. Moreover, its iterated derivative satisfies

$$
\begin{aligned}
& D_{r_{1}, v_{1}}^{\left(k_{1}\right)} \cdots D_{r_{n}, v_{n}}^{\left(k_{n}\right)}\left(u_{i}(t, x)\right) \\
& =\sum_{l=1}^{d} G_{t-r_{l}}\left(x, v_{l}\right)\left(D_{r_{1}, v_{1}}^{\left(k_{1}\right)} \cdots D_{r_{l-1}, v_{l-1}}^{\left(k_{l-1}\right)} D_{r_{l+1}, v_{l+1}}^{\left(k_{l+1}\right)} \cdots D_{r_{n}, v_{n}}^{\left(k_{n}\right)}\left(\sigma_{i k_{l}}\left(u\left(r_{l}, v_{l}\right)\right)\right)\right) \\
& \quad+\sum_{j=1}^{d} \int_{r_{1} \vee \cdots \vee r_{n}}^{t} \int_{0}^{1} G_{t-\theta}(x, \eta) \prod_{l=1}^{n} D_{r_{l}, v_{l}}^{\left(k_{l}\right)}\left(\sigma_{i j}(u(\theta, \eta))\right) W^{j}(d \theta, d \eta) \\
& \quad+\int_{r_{1} \vee \cdots \vee r_{n}}^{t} \int_{0}^{1} G_{t-\theta}(x, \eta) \prod_{l=1}^{n} D_{r_{l}, v_{l}}^{\left(k_{l}\right)}\left(b_{i}(u(\theta, \eta))\right) d \theta d \eta
\end{aligned}
$$

if $t \leq r_{1} \vee \cdots \vee r_{n}$ and $D_{r_{1}, v_{1}}^{\left(k_{1}\right)} \cdots D_{r_{n}, v_{n}}^{\left(k_{n}\right)}\left(u_{i}(t, x)\right)=0$ otherwise. Finally, for any $p>1$,

$$
\sup _{(t, x) \in[0, T] \times[0,1]} \mathrm{E}\left[\left\|D^{n}\left(u_{i}(t, x)\right)\right\|_{\mathscr{H}^{\otimes n}}^{p}\right]<+\infty .
$$

Note that, in particular, the first-order Malliavin derivative satisfies, for $r<t$,

$$
D_{r, v}^{(k)}\left(u_{i}(t, x)\right)=G_{t-r}(x, v) \sigma_{i k}(u(r, v))+a_{i}(k, r, v, t, x),
$$

where

$$
\begin{aligned}
a_{i}(k, r, v, t, x)=\sum_{j=1}^{d} \int_{r}^{t} & \int_{0}^{1} G_{t-\theta}(x, \eta) D_{r, v}^{(k)}\left(\sigma_{i j}(u(\theta, \eta))\right) W^{j}(d \theta, d \eta) \\
& +\int_{r}^{t} \int_{0}^{1} G_{t-\theta}(x, \eta) D_{r, v}^{(k)}\left(b_{i}(u(\theta, \eta))\right) d \theta d \eta
\end{aligned}
$$

and $D_{r, v}^{(k)}\left(u_{i}(t, x)\right)=0$ when $r>t$.

The next result proves property (a) in Proposition 3.4 when $F$ is replaced by $u(t, x)$.

Proposition 4.2. Assume $\mathbf{P} 1$ and $\mathbf{P 2}$. Let $I$ and $J$ be two compact intervals as in Theorem 1.1. Then, for any $p \geq 1$,

$$
\mathrm{E}\left[\left(\operatorname{det} \gamma_{u(t, x)}\right)^{-p}\right]
$$

is uniformly bounded over $(t, x) \in I \times J$.

Proof. This proof follows Nualart [N98, Proof of (3.22)], where it is shown that for fixed $(t, x), \mathrm{E}\left[\left(\operatorname{det} \gamma_{u(t, x)}\right)^{-p}\right]<+\infty$. Our emphasis here is on the uniform bound over $(t, x) \in$ $I \times J$. Assume that $I=\left[t_{1}, t_{2}\right]$ and $J=\left[x_{1}, x_{2}\right]$, where $0<t_{1}<t_{2} \leq T, 0<x_{1}<x_{2}<1$. Let $(t, x) \in I \times J$ be fixed. We write

$$
\operatorname{det} \gamma_{u(t, x)} \geq\left(\inf _{\xi \in \mathbb{R}^{d}:\|\xi\|=1} \xi^{T} \gamma_{u(t, x)} \xi\right)^{d}
$$


Let $\xi=\left(\xi_{1}, \ldots, \xi_{d}\right) \in \mathbb{R}^{d}$ with $\|\xi\|=1$ and fix $\epsilon \in(0,1)$. Note the inequality

$$
(a+b)^{2} \geq \frac{2}{3} a^{2}-2 b^{2},
$$

valid for all $a, b \in \mathbb{R}$. Using (4.2) and the fact that $\gamma_{u(t, x)}$ is a matrix whose entries are inner-products, this implies that

$$
\begin{aligned}
\xi^{T} \gamma_{u(t, x)} \xi & =\int_{0}^{t} d r \int_{0}^{1} d v\left\|\mid \sum_{i=1}^{d} D_{r, v}\left(u_{i}(t, x)\right) \xi_{i}\right\|^{2} \\
& \geq \int_{t(1-\epsilon)}^{t} d r \int_{0}^{1} d v\left\|\sum_{i=1}^{d} D_{r, v}\left(u_{i}(t, x)\right) \xi_{i}\right\|^{2} \geq I_{1}-I_{2},
\end{aligned}
$$

where

$$
\begin{aligned}
& I_{1}=\frac{2}{3} \int_{t(1-\epsilon)}^{t} d r \int_{0}^{1} d v \sum_{k=1}^{d}\left(\sum_{i=1}^{d} G_{t-r}(x, v) \sigma_{i k}(u(r, v)) \xi_{i}\right)^{2}, \\
& I_{2}=2 \int_{t(1-\epsilon)}^{t} d r \int_{0}^{1} d v \sum_{k=1}^{d}\left(\sum_{i=1}^{d} a_{i}(k, r, v, t, x) \xi_{i}\right)^{2},
\end{aligned}
$$

and $a_{i}(k, r, v, t, x)$ is defined in (4.3). In accord with hypothesis $\mathbf{P 2}$ and thanks to Lemma 7.2 ,

$$
I_{1} \geq c(t \epsilon)^{1 / 2}
$$

where $c$ is uniform over $(t, x) \in I \times J$.

Next we apply the Cauchy-Schwarz inequality to find that, for any $q \geq 1$,

$$
\mathrm{E}\left[\sup _{\xi \in \mathbb{R}^{d}:\|\xi\|=1}\left|I_{2}\right|^{q}\right] \leq c\left(\mathrm{E}\left[\left|A_{1}\right|^{q}\right]+\mathrm{E}\left[\left|A_{2}\right|^{q}\right]\right)
$$

where

$$
\begin{aligned}
& A_{1}=\sum_{i, j, k=1}^{d} \int_{t(1-\epsilon)}^{t} d r \int_{0}^{1} d v\left(\int_{r}^{t} \int_{0}^{1} G_{t-\theta}(x, \eta) D_{r, v}^{(k)}\left(\sigma_{i j}(u(\theta, \eta))\right) W^{j}(d \theta, d \eta)\right)^{2}, \\
& A_{2}=\sum_{i, k=1}^{d} \int_{t(1-\epsilon)}^{t} d r \int_{0}^{1} d v\left(\int_{r}^{t} \int_{0}^{1} G_{t-\theta}(x, \eta) D_{r, v}^{(k)}\left(b_{i}(u(\theta, \eta))\right) d \theta d \eta\right)^{2} .
\end{aligned}
$$

We bound the $q$-th moment of $A_{1}$ and $A_{2}$ separately. As regards $A_{1}$, we use Burkholder's inequality for martingales with values in a Hilbert space (Lemma 7.6) to obtain

$$
\mathrm{E}\left[\left|A_{1}\right|^{q}\right] \leq c \sum_{k, i=1}^{d} \mathrm{E}\left[\left|\int_{t(1-\epsilon)}^{t} d \theta \int_{0}^{1} d \eta \int_{t(1-\epsilon)}^{t} d r \int_{0}^{1} d v \Theta^{2}\right|^{q}\right],
$$


where

$$
\begin{aligned}
\Theta & :=1_{\{\theta>r\}} G_{t-\theta}(x, \eta)\left|D_{r, v}^{(k)}\left(\sigma_{i j}(u(\theta, \eta))\right)\right| \\
& \leq c 1_{\{\theta>r\}} G_{t-\theta}(x, \eta)\left|\sum_{l=1}^{d} D_{r, v}^{(k)}\left(u_{l}(\theta, \eta)\right)\right|,
\end{aligned}
$$

thanks to hypothesis $\mathbf{P} 1$. Hence,

$$
\mathrm{E}\left[\left|A_{1}\right|^{q}\right] \leq c \sum_{k=1}^{d} \mathrm{E}\left[\left|\int_{t(1-\epsilon)}^{t} d \theta \int_{0}^{1} d \eta G_{t-\theta}^{2}(x, \eta) \int_{t(1-\epsilon)}^{t \wedge \theta} d r \int_{0}^{1} d v \Psi^{2}\right|^{q}\right],
$$

where $\Psi:=\sum_{l=1}^{d} D_{r, v}^{(k)}\left(u_{l}(\theta, \eta)\right)$. We now apply Hölder's inequality with respect to the measure $G_{t-\theta}^{2}(x, \eta) d \theta d \eta$ to find that

$$
\begin{aligned}
\mathrm{E}\left[\left|A_{1}\right|^{q}\right] \leq C\left|\int_{t(1-\epsilon)}^{t} d \theta \int_{0}^{1} d \eta G_{t-\theta}^{2}(x, \eta)\right|^{q-1} \\
\quad \times \int_{t(1-\epsilon)}^{t} d \theta \int_{0}^{1} d \eta G_{t-\theta}^{2}(x, \eta) \sum_{k=1}^{d} \mathrm{E}\left[\left|\int_{t(1-\epsilon)}^{t} d r \int_{0}^{1} d v \Psi^{2}\right|^{q}\right] .
\end{aligned}
$$

Lemmas 7.3 and 7.5 assure that

$$
\mathrm{E}\left[\left|A_{1}\right|^{q}\right] \leq C_{T}(t \epsilon)^{\frac{q-1}{2}}(t \epsilon)^{q / 2} \int_{t(1-\epsilon)}^{t} \int_{0}^{1} G_{t-\theta}^{2}(x, \eta) d \theta d \eta \leq C_{T}(t \epsilon)^{q},
$$

where $C_{T}$ is uniform over $(t, x) \in I \times J$.

We next derive a similar bound for $A_{2}$. By the Cauchy-Schwarz inequality,

$$
\mathrm{E}\left[\left|A_{2}\right|^{q}\right] \leq c(t \epsilon)^{q} \sum_{i, k=1}^{d} \mathrm{E}\left[\left|\int_{t(1-\epsilon)}^{t} d r \int_{0}^{1} d v \int_{r}^{t} d \theta \int_{0}^{1} d \eta \Phi^{2}\right|^{q}\right],
$$

where $\Phi:=G_{t-\theta}(x, \eta)\left|D_{r, v}^{(k)}\left(b_{i}(u(\theta, \eta))\right)\right|$. From here on, the $q$-th moment of $A_{2}$ is estimated as that of $A_{1}$ was; cf. (4.6), and this yields $\mathrm{E}\left[\left|A_{2}\right|^{q}\right] \leq C_{T}(t \epsilon)^{2 q}$.

Thus, we have proved that

$$
\mathrm{E}\left[\sup _{\xi \in \mathbb{R}^{d}:\|\xi\|=1}\left|I_{2}\right|^{q}\right] \leq C_{T}(t \epsilon)^{q},
$$

where the constant $C_{T}$ is clearly uniform over $(t, x) \in I \times J$.

Finally, we apply Proposition 3.5 with $Z:=\inf _{\|\xi\|=1}\left(\xi^{T} \gamma_{u(t, x)} \xi\right), Y_{1, \epsilon}=Y_{2, \epsilon}=\sup _{\|\xi\|=1} I_{2}$, $\epsilon_{0}=1, \alpha_{1}=\alpha_{2}=1 / 2$ and $\beta_{1}=\beta_{2}=1$, to get

$$
\mathrm{E}\left[\left(\operatorname{det} \gamma_{u(t, x)}\right)^{-p}\right] \leq C_{T}
$$

where all the constants are clearly uniform over $(t, x) \in I \times J$. This is the desired result. 
The two previous propositions have the following corollary.

Corollary 4.3. Assume $\mathbf{P} 1$ and $\mathbf{P 2}$. Fix $T>0$ and let $I$ and $J$ be a compact intervals as in Theorem 1.1. Then, for any $(t, x) \in(0, T] \times(0,1), u(t, x)$ is a nondegenerate random vector and its density function is infinitely differentiable and uniformly bounded over $z \in \mathbb{R}^{d}$ and $(t, x) \in I \times J$.

Proof. The conclusions are a consequence of Propositions 4.1 and 4.2 together with Theorem 3.1 and Proposition 3.4.

Proof of Theorem 1.1(a). This is an immediate consequence of Corollary 4.3.

\section{The Gaussian-type lower bound on the one-point density}

The aim of this section is to prove the lower bound of Gaussian-type for the density of $u$ stated in Theorem 1.1(b). The proof of this result was given in KoHATsu-Higa [K03, Theorem 10] for dimension 1 , therefore we will only sketch the main steps.

Proof of Theorem 1.1(b). We follow [K03] and we show that for each $(t, x), F=u(t, x)$ is a $d$-dimensional uniformly elliptic random vector and then we apply [K03, Theorem 5]. Let

$$
F_{n}^{i}=\int_{0}^{t_{n}} \int_{0}^{1} G_{t-r}(x, v) \sum_{j=1}^{d} \sigma_{i j}(u(r, v)) W^{j}(d r, d v)+\int_{0}^{t_{n}} \int_{0}^{1} G_{t-r}(x, v) b_{i}(u(r, v)) d r d v,
$$

$1 \leq i \leq d$, where $0=t_{0}<t_{1}<\cdots<t_{N}=t$ is a sufficiently fine partition of $[0, t]$. Note that $F_{n} \in \mathscr{F}_{t_{n}}$. Set $g(s, y)=G_{t-s}(x, y)$. We shall need the following two lemmas.

Lemma 5.1. [K03, Lemma 7] Assume $\mathbf{P 1}$ and P2. Then:

(i) $\left\|F_{n}^{i}\right\|_{k, p} \leq c_{k, p}, 1 \leq i \leq d ;$

(ii) $\left\|\left(\left(\gamma_{F_{n}}\left(t_{n-1}\right)\right)_{i j}\right)^{-1}\right\|_{p, t_{n-1}} \leq c_{p}\left(\Delta_{n-1}(g)\right)^{-1}=c_{p}\left(\|g\|_{L^{2}\left(\left[t_{n-1}, t_{n}\right] \times[0,1]\right)}^{2}\right)^{-1}$,

where $\gamma_{F_{n}}\left(t_{n-1}\right)$ denotes the conditional Malliavin matrix of $F_{n}$ given $\mathscr{F}_{t_{n-1}}$ and $\|\cdot\|_{p, t_{n-1}}$ denotes the conditional $L^{p}$-norm.

We define

$$
\begin{aligned}
u_{i}^{n-1}\left(s_{1}, y_{1}\right)= & \int_{0}^{t_{n-1}} \int_{0}^{1} G_{s_{1}-s_{2}}\left(y_{1}, y_{2}\right) \sum_{j=1}^{d} \sigma_{i j}\left(u\left(s_{2}, y_{2}\right)\right) W^{j}\left(d s_{1}, d y_{2}\right) \\
& +\int_{0}^{t_{n-1}} \int_{0}^{1} G_{s_{1}-s_{2}}\left(y_{1}, y_{2}\right) b_{i}\left(u\left(s_{2}, y_{2}\right)\right) d s_{2} d y_{2}, \quad 1 \leq i \leq d .
\end{aligned}
$$

Note that $u^{n-1} \in \mathscr{F}_{t_{n-1}}$. As in [K03], the following holds. 
Lemma 5.2. [K03, Lemma 8] Under hypothesis $\mathbf{P} \mathbf{1}$, for $s \in\left[t_{n-1}, t_{n}\right]$,

$$
\left\|u_{i}(s, y)-u_{i}^{n-1}(s, y)\right\|_{n, p, t_{n-1}} \leq\left(s-t_{n-1}\right)^{1 / 8}, \quad 1 \leq i \leq d,
$$

where $\|\cdot\|_{n, p, t_{n-1}}$ denotes the conditional Malliavin norm given $\mathscr{F}_{t_{n-1}}$.

The rest of the proof of Theorem 1.1(b) follows along the same lines as in [K03] for $d=1$. We only sketch the remaining main points where the fact that $d>1$ is important. In order to obtain the expansion of $F_{n}^{i}-F_{n-1}^{i}$ as in [K03, Lemma 9], we proceed as follows. By the mean value theorem,

$$
\begin{aligned}
F_{n}^{i}- & F_{n-1}^{i}=\int_{t_{n-1}}^{t_{n}} \int_{0}^{1} G_{t-r}(x, v) \sum_{j=1}^{d} \sigma_{i j}\left(u^{n-1}(r, v)\right) W^{j}(d r, d v) \\
& +\int_{t_{n-1}}^{t_{n}} \int_{0}^{1} G_{t-r}(x, v) b_{i}(u(r, v)) d r d v \\
& +\int_{t_{n-1}}^{t_{n}} \int_{0}^{1} G_{t-r}(x, v) \sum_{j, l=1}^{d}\left(\int_{0}^{1} \partial_{l} \sigma_{i j}(u(r, v, \lambda)) d \lambda\right)\left(u_{l}(r, v)-u_{l}^{n-1}(r, v)\right) W^{j}(d r, d v),
\end{aligned}
$$

where $u(r, v, \lambda)=(1-\lambda) u(r, v)+\lambda u^{n-1}(r, v)$. Using the terminology of [K03], the first term is a process of order 1 and the next two terms are residues of order 1 (as in [K03]). In the next step, we write the residues of order 1 as the sum of processes of order 2 and residues of order 2 and 3 as follows:

$$
\begin{aligned}
\int_{t_{n-1}}^{t_{n}} & \int_{0}^{1} G_{t-r}(x, v) b_{i}(u(r, v)) d r d v \\
= & \int_{t_{n-1}}^{t_{n}} \int_{0}^{1} G_{t-r}(x, v) b_{i}\left(u^{n-1}(r, v)\right) d r d v \\
& \quad+\int_{t_{n-1}}^{t_{n}} \int_{0}^{1} G_{t-r}(x, v) \sum_{l=1}^{d}\left(\int_{0}^{1} \partial_{l} b_{i}(u(r, v, \lambda)) d \lambda\right)\left(u_{l}(r, v)-u_{l}^{n-1}(r, v)\right) d r d v
\end{aligned}
$$

and

$$
\begin{aligned}
& \int_{t_{n-1}}^{t_{n}} \int_{0}^{1} G_{t-r}(x, v) \sum_{j, l=1}^{d}\left(\int_{0}^{1} \partial_{l} \sigma_{i j}(u(r, v, \lambda)) d \lambda\right)\left(u_{l}(r, v)-u_{l}^{n-1}(r, v)\right) W^{j}(d r, d v) \\
& =\int_{t_{n-1}}^{t_{n}} \int_{0}^{1} G_{t-r}(x, v) \sum_{j, l=1}^{d} \partial_{l} \sigma_{i j}\left(u^{n-1}(r, v)\right)\left(u_{l}(r, v)-u_{l}^{n-1}(r, v)\right) W^{j}(d r, d v) \\
& \quad+\int_{t_{n-1}}^{t_{n}} \int_{0}^{1} G_{t-r}(x, v) \sum_{j, l, l^{\prime}=1}^{d}\left(\int_{0}^{1} \partial_{l} \partial_{l^{\prime}} \sigma_{i j}(u(r, v, \lambda)) d \lambda\right) \\
& \quad \times\left(u_{l}(r, v)-u_{l}^{n-1}(r, v)\right)\left(u_{l^{\prime}}(r, v)-u_{l^{\prime}}^{n-1}(r, v)\right) W^{j}(d r, d v) .
\end{aligned}
$$

It is then clear that the remainder of the proof of [K03, Lemma 9] follows for $d>1$ along the same lines as in [K03], working coordinate by coordinate. 
Finally, in order to complete the proof of the proposition, it suffices to verify the hypotheses of [K03, Theorem 5]. Again the proof follows as in the proof of [K03, Theorem 10], working coordinate by coordinate. We will only sketch the proof of his (H2c), where hypothesis $\mathbf{P 2}$ is used:

$$
\begin{aligned}
& \left(\Delta_{n-1}(g)\right)^{-1} \int_{t_{n-1}}^{t_{n}} \int_{0}^{1}\left(G_{t-r}(x, v)\right)^{2}\left\|\sigma\left(u^{n-1}(r, v)\right) \xi\right\|^{2} d r d v \\
& \geq \rho^{2}\left(\Delta_{n-1}(g)\right)^{-1} \int_{t_{n-1}}^{t_{n}} \int_{0}^{1}\left(G_{t-r}(x, v)\right)^{2} d r d v=\rho^{2}>0
\end{aligned}
$$

by the definition of $g$. This concludes the proof of Theorem 1.1 (b).

\section{The Gaussian-type upper bound on the two-point density}

Let $p_{s, y ; t, x}\left(z_{1}, z_{2}\right)$ denote the joint density of the $2 d$-dimensional random vector

$$
\left(u_{1}(s, y), \ldots, u_{d}(s, y), u_{1}(t, x), \ldots, u_{d}(t, x)\right),
$$

for $s, t \in(0, T], x, y \in(0,1),(s, y) \neq(t, x)$ and $z_{1}, z_{2} \in \mathbb{R}^{d}$ (the existence of this joint density will be a consequence of Theorem 3.1, Proposition 4.1 and Theorem 6.3).

The next subsections lead to the proofs of Theorem 1.1(c) and (d).

\subsection{Bounds on the increments of the Malliavin derivatives}

In this subsection, we prove an upper bound for the Sobolev norm of the derivative of the increments of our process $u$. For this, we will need the following preliminary estimate.

Lemma 6.1. For any $s, t \in[0, T], s \leq t$, and $x, y \in[0,1]$,

$$
\int_{0}^{T} \int_{0}^{1}(g(r, v))^{2} d r d v \leq C_{T}\left(|t-s|^{1 / 2}+|x-y|\right),
$$

where

$$
g(r, v):=g_{t, x, s, y}(r, v)=1_{\{r \leq t\}} G_{t-r}(x, v)-1_{\{r \leq s\}} G_{s-r}(y, v) .
$$

Proof. Using Bally, Millet, And Sanz-Solé [BMS95, Lemma B.1] with $\alpha=2$, we see that

$$
\begin{aligned}
& \int_{0}^{T} \int_{0}^{1}(g(r, v))^{2} d r d v \\
& \leq \int_{s}^{t} \int_{0}^{1}\left(G_{t-r}(x, v)\right)^{2} d r d v+2 \int_{0}^{s} \int_{0}^{1}\left(G_{t-r}(x, v)-G_{s-r}(x, v)\right)^{2} d r d v \\
& \quad+2 \int_{0}^{s} \int_{0}^{1}\left(G_{s-r}(x, v)-G_{s-r}(y, v)\right)^{2} d r d v \\
& \leq C_{T}\left(|t-s|^{1 / 2}+|x-y|\right) .
\end{aligned}
$$


Proposition 6.2. Assuming P1, for any $s, t \in[0, T], s \leq t, x, y \in[0,1], p>1, m \geq 1$,

$$
\mathrm{E}\left[\left\|D^{m}\left(u_{i}(t, x)-u_{i}(s, y)\right)\right\|_{\mathscr{H} \otimes m}^{p}\right] \leq C_{T}\left(|t-s|^{1 / 2}+|x-y|\right)^{p / 2}, \quad i=1, \ldots, d .
$$

Proof. Let $m=1$. Consider the function $g(r, v)$ defined in Lemma 6.1. Using the integral equation (4.2) satisfied by the first-order Malliavin derivative, we find that

$$
\mathrm{E}\left[\left\|D\left(u_{i}(t, x)-u_{i}(s, y)\right)\right\|_{\mathscr{H}}^{p}\right] \leq C\left(\mathrm{E}\left[\left|I_{1}\right|^{p / 2}\right]+\mathrm{E}\left[\left|I_{2}\right|^{p / 2}\right]+\mathrm{E}\left[\left|I_{3}\right|^{p / 2}\right]\right),
$$

where

$$
\begin{aligned}
& I_{1}=\sum_{k=1}^{d} \int_{0}^{T} d r \int_{0}^{1} d v\left(g(r, v) \sigma_{i k}(u(r, v))\right)^{2}, \\
& I_{2}=\sum_{j, k=1}^{d} \int_{0}^{T} d r \int_{0}^{1} d v\left(\int_{0}^{T} \int_{0}^{1} g(\theta, \eta) D_{r, v}^{(k)}\left(\sigma_{i j}(u(\theta, \eta))\right) W^{j}(d \theta, d \eta)\right)^{2}, \\
& I_{3}=\sum_{k=1}^{d} \int_{0}^{T} d r \int_{0}^{1} d v\left(\int_{0}^{T} \int_{0}^{1} g(\theta, \eta) D_{r, v}^{(k)}\left(b_{i}(u(\theta, \eta))\right) d \theta d \eta\right)^{2} .
\end{aligned}
$$

We bound the $p / 2$-moments of $I_{1}, I_{2}$ and $I_{3}$ separately.

By hypothesis $\mathbf{P 1}$ and Lemma $6.1, E\left[\left|I_{1}\right|^{p / 2}\right] \leq C_{T}\left(|t-s|^{1 / 2}+|x-y|\right)^{p / 2}$. Using Burkholder's inequality for Hilbert-space-valued martingales (Lemma 7.6) and hypothesis P1, we obtain

$$
\mathrm{E}\left[\left|I_{2}\right|^{p / 2}\right] \leq C \sum_{k=1}^{d} \mathrm{E}\left[\left|\int_{0}^{T} d \theta \int_{0}^{1} d \eta(g(\theta, \eta))^{2} \int_{0}^{T} d r \int_{0}^{1} d v \Theta^{2}\right|^{p / 2}\right]
$$

where $\Theta:=\sum_{l=1}^{d} D_{r, v}^{(k)}\left(u_{l}(\theta, \eta)\right)$. From Hölder's inequality with respect to the measure $(g(\theta, \eta))^{2} d \theta d \eta$, we see that this is bounded above by

$$
\begin{aligned}
& C\left(\int_{0}^{T} \int_{0}^{1}(g(\theta, \eta))^{2} d \theta d \eta\right)^{\frac{p}{2}-1} \\
& \quad \times \sup _{(\theta, \eta) \in[0, T] \times[0,1]} \sum_{k=1}^{d} \mathrm{E}\left[\left|\int_{0}^{T} d \theta \int_{0}^{1} d \eta(g(\theta, \eta))^{2} \int_{0}^{T} d r \int_{0}^{1} d v \Theta^{2}\right|^{p / 2}\right] \\
& \leq C_{T}\left(|t-s|^{1 / 2}+|x-y|\right)^{p / 2}
\end{aligned}
$$

thanks to (4.1) and Lemma 6.1.

We next derive a similar bound for $I_{3}$. By the Cauchy-Schwarz inequality,

$$
\mathrm{E}\left[\left|I_{3}\right|^{p / 2}\right] \leq C_{T} \sum_{k=1}^{d} \mathrm{E}\left[\left|\int_{0}^{T} d \theta \int_{0}^{1} d \eta(g(\theta, \eta))^{2} \int_{0}^{T} d r \int_{0}^{1} d v \Theta^{2}\right|^{p / 2}\right] .
$$


From here on, the $p / 2$-moment of $I_{3}$ is estimated as was that of $I_{2}$, and this yields $\mathrm{E}\left[\left|I_{3}\right|^{p / 2}\right] \leq$ $C_{T}\left(|t-s|^{1 / 2}+|x-y|\right)^{p / 2}$. This proves the desired result for $m=1$. The case $m>1$ follows using the stochastic differential equation satisfied by the iterated Malliavin derivatives (Proposition 4.1), Hölder's and Burkholder's inequalities, hypothesis P1, (4.1) and Lemma 6.1 in the same way as we $\operatorname{did}$ for $m=1$, to obtain the desired bound.

\subsection{Study of the Malliavin matrix}

For $s, t \in[0, T], s \leq t$, and $x, y \in[0,1]$ consider the $2 d$-dimensional random vector

$$
Z:=\left(u_{1}(s, y), \ldots, u_{d}(s, y), u_{1}(t, x)-u_{1}(s, y), \ldots, u_{d}(t, x)-u_{d}(s, y)\right) .
$$

Let $\gamma_{Z}$ the Malliavin matrix of $Z$. Note that $\gamma_{Z}=\left(\left(\gamma_{Z}\right)_{m, l}\right)_{m, l=1, \ldots, 2 d}$ is a symmetric $2 d \times 2 d$ random matrix with four $d \times d$ blocs of the form

$$
\gamma_{Z}=\left(\begin{array}{ccc}
\gamma_{Z}^{(1)} & \vdots & \gamma_{Z}^{(2)} \\
\cdots & \vdots & \cdots \\
\gamma_{Z}^{(3)} & \vdots & \gamma_{Z}^{(4)}
\end{array}\right)
$$

where

$$
\begin{aligned}
\gamma_{Z}^{(1)} & =\left(\left\langle D\left(u_{i}(s, y)\right), D\left(u_{j}(s, y)\right)\right\rangle_{\mathscr{H}}\right)_{i, j=1, \ldots, d} \\
\gamma_{Z}^{(2)} & =\left(\left\langle D\left(u_{i}(s, y)\right), D\left(u_{j}(t, x)-u_{j}(s, y)\right)\right\rangle_{\mathscr{H}}\right)_{i, j=1, \ldots, d}, \\
\gamma_{Z}^{(3)} & =\left(\left\langle D\left(u_{i}(t, x)-u_{i}(s, y)\right), D\left(u_{j}(s, y)\right)\right\rangle_{\mathscr{H}}\right)_{i, j=1, \ldots, d}, \\
\gamma_{Z}^{(4)} & =\left(\left\langle D\left(u_{i}(t, x)-u_{i}(s, y)\right), D\left(u_{j}(t, x)-u_{j}(s, y)\right)\right\rangle_{\mathscr{H}}\right)_{i, j=1, \ldots, d}
\end{aligned}
$$

We let (1) denote the set of indices $\{1, \ldots, d\} \times\{1, \ldots, d\},(2)$ the set $\{1, \ldots, d\} \times\{d+1, \ldots, 2 d\}$, (3) the set $\{d+1, \ldots, 2 d\} \times\{1, \ldots, d\}$ and (4) the set $\{d+1, \ldots, 2 d\} \times\{d+1, \ldots, 2 d\}$.

The following theorem gives an estimate on the Sobolev norm of the entries of the inverse of the matrix $\gamma_{Z}$, which depends on the position of the entry in the matrix.

Theorem 6.3. Fix $\eta, T>0$. Assume $\mathbf{P 1}$ and $\mathbf{P 2}$. Let $I$ and $J$ be two compact intervals as in Theorem 1.1.

(a) For any $(s, y) \in I \times J,(t, x) \in I \times J, s \leq t,(s, y) \neq(t, x), k \geq 0, p>1$,

$$
\left\|\left(\gamma_{Z}^{-1}\right)_{m, l}\right\|_{k, p} \leq\left\{\begin{array}{lll}
c_{k, p, \eta, T}\left(|t-s|^{1 / 2}+|x-y|\right)^{-d \eta} & \text { if } \quad(m, l) \in(\mathbf{1}), \\
c_{k, p, \eta, T}\left(|t-s|^{1 / 2}+|x-y|\right)^{-1 / 2-d \eta} & \text { if } \quad(m, l) \in(\mathbf{2}) \text { or }(\mathbf{3}), \\
c_{k, p, \eta, T}\left(|t-s|^{1 / 2}+|x-y|\right)^{-1-d \eta} & \text { if } \quad(m, l) \in(\mathbf{4})
\end{array}\right.
$$

(b) For any $s=t \in(0, T],(t, y) \in I \times J,(t, x) \in I \times J, x \neq y, k \geq 0, p>1$,

$$
\left\|\left(\gamma_{Z}^{-1}\right)_{m, l}\right\|_{k, p} \leq \begin{cases}c_{k, p, T} & \text { if } \quad(m, l) \in(\mathbf{1}), \\ c_{k, p, T}|x-y|^{-1 / 2} & \text { if } \quad(m, l) \in(\mathbf{2}) \text { or }(\mathbf{3}), \\ c_{k, p, T}|x-y|^{-1} & \text { if } \quad(m, l) \in(\mathbf{4}) .\end{cases}
$$


(Note the slight improvements in the exponents in case (b) where $s=t$. )

The proof of this theorem is deferred to Section 6.4. We assume it for the moment and complete the proof of Theorem 1.1(c) and (d).

\subsection{Proof of Theorem 1.1(c) and (d)}

Fix two compact intervals $I$ and $J$ as in Theorem 1.1. Let $(s, y),(t, x) \in I \times J, s \leq t$, $(s, y) \neq(t, x)$, and $z_{1}, z_{2} \in \mathbb{R}^{d}$. Let $Z$ be as in (6.1) and let $p_{Z}$ be the density of $Z$. Then

$$
p_{s, y ; t, x}\left(z_{1}, z_{2}\right)=p_{Z}\left(z_{1}, z_{1}-z_{2}\right) .
$$

Apply Corollary 3.3 with $\sigma=\left\{i \in\{1, \ldots, d\}: z_{1}^{i}-z_{2}^{i} \geq 0\right\}$ and Hölder's inequality to see that

$$
p_{Z}\left(z_{1}, z_{1}-z_{2}\right) \leq \prod_{i=1}^{d}\left(\mathrm{P}\left\{\left|u_{i}(t, x)-u_{i}(s, y)\right|>\left|z_{1}^{i}-z_{2}^{i}\right|\right\}\right)^{\frac{1}{2 d}} \times\left\|H_{(1, \ldots, 2 d)}(Z, 1)\right\|_{0,2} .
$$

Therefore, in order to prove the desired results (c) and (d) of Theorem 1.1, it suffices to prove that:

$$
\begin{gathered}
\prod_{i=1}^{d}\left(\mathrm{P}\left\{\left|u_{i}(t, x)-u_{i}(s, y)\right|>\left|z_{1}^{i}-z_{2}^{i}\right|\right\}\right)^{\frac{1}{2 d}} \leq c \exp \left(-\frac{\left\|z_{1}-z_{2}\right\|^{2}}{c_{T}\left(|t-s|^{1 / 2}+|x-y|\right)}\right) \\
\left\|H_{(1, \ldots, 2 d)}(Z, 1)\right\|_{0,2} \leq c_{T}\left(|t-s|^{1 / 2}+|x-y|\right)^{-(d+\eta) / 2}
\end{gathered}
$$

and if $s=t$, then

$$
\left\|H_{(1, \ldots, 2 d)}(Z, 1)\right\|_{0,2} \leq c_{T}|x-y|^{-d / 2}
$$

Proof of (6.2). Let $\tilde{u}$ denote the solution of (2.2) for $b \equiv 0$. Consider the continuous oneparameter martingale $\left(M_{u}=\left(M_{u}^{1}, \ldots, M_{u}^{d}\right), 0 \leq u \leq t\right)$ defined by

$$
M_{u}^{i}=\left\{\begin{array}{cc}
\int_{0}^{u} \int_{0}^{1}\left(G_{t-r}(x, v)-G_{s-r}(y, v)\right) \sum_{j=1}^{d} \sigma_{i j}(\tilde{u}(r, v)) W^{j}(d r, d v) & \text { if } 0 \leq u \leq s, \\
\int_{0}^{s} \int_{0}^{1}\left(G_{t-r}(x, v)-G_{s-r}(y, v)\right) \sum_{j=1}^{d} \sigma_{i j}(\tilde{u}(r, v)) W^{j}(d r, d v) & \\
+\int_{s}^{u} \int_{0}^{1} G_{t-r}(x, v) \sum_{j=1}^{d} \sigma_{i j}(\tilde{u}(r, v)) W^{j}(d r, d v) & \text { if } s \leq u \leq t,
\end{array}\right.
$$

for all $i=1, \ldots, d$, with respect to the filtration $\left(\mathscr{F}_{u}, 0 \leq u \leq t\right)$. Notice that

$$
M_{0}=0, \quad M_{t}=\tilde{u}(t, x)-\tilde{u}(s, y) .
$$


Moreover, by hypothesis P1 and Lemma 6.1,

$$
\begin{aligned}
\left\langle M^{i}\right\rangle_{t} & =\int_{0}^{s} \int_{0}^{1}\left(G_{t-r}(x, v)-G_{s-r}(y, v)\right)^{2} \sum_{j=1}^{d}\left(\sigma_{i j}(\tilde{u}(r, v))\right)^{2} d r d v \\
& \quad+\int_{s}^{t} \int_{0}^{1}\left(G_{t-r}(x, v)\right)^{2} \sum_{j=1}^{d}\left(\sigma_{i j}(\tilde{u}(r, v))\right)^{2} d r d v \\
& \leq C \int_{0}^{T} \int_{0}^{1}(g(r, v))^{2} d r d v \\
\leq & C_{T}\left(|t-s|^{1 / 2}+|x-y|\right) .
\end{aligned}
$$

By the exponential martingale inequality Nualart [N95, A.5],

$$
\mathrm{P}\left\{\left|\tilde{u}_{i}(t, x)-\tilde{u}_{i}(s, y)\right|>\left|z_{1}^{i}-z_{2}^{i}\right|\right\} \leq 2 \exp \left(-\frac{\left|z_{1}^{i}-z_{2}^{i}\right|^{2}}{C_{T}\left(|t-s|^{1 / 2}+|x-y|\right)}\right) .
$$

We will now treat the case $b \not \equiv 0$ using Girsanov's theorem. Consider the random variable

$$
\begin{aligned}
L_{t}=\exp \left(-\int_{0}^{t} \int_{0}^{1} \sigma^{-1}(u(r, v)) b(u(r, v)) \cdot W(d r, d v)\right. \\
\left.\quad-\frac{1}{2} \int_{0}^{t} \int_{0}^{1}\left\|\sigma^{-1}(u(r, v)) b(u(r, v))\right\|^{2} d r d v\right) .
\end{aligned}
$$

The following Girsanov's theorem holds.

Theorem 6.4. [NP94, Prop.1.6] $\mathrm{E}\left[L_{t}\right]=1$, and if $\tilde{P}$ denotes the probability measure on $(\Omega, \mathscr{F})$ defined by

$$
\frac{d \tilde{\mathrm{P}}}{d \mathrm{P}}(\omega)=L_{t}(\omega)
$$

then $\tilde{W}(t, x)=W(t, x)+\int_{0}^{t} \int_{0}^{x} \sigma^{-1}(u(r, v)) b(u(r, v)) d r d v$ is a standard Brownian sheet under $\tilde{\mathrm{P}}$.

Consequently, the law of $u$ under $\tilde{\mathrm{P}}$ coincides with the law of $\tilde{u}$ under $\mathrm{P}$. Consider now the random variable

$$
\begin{array}{r}
J_{t}=\exp \left(-\int_{0}^{t} \int_{0}^{1} \sigma^{-1}(\tilde{u}(r, v)) b(\tilde{u}(r, v)) \cdot W(d r, d v)\right. \\
\left.+\frac{1}{2} \int_{0}^{t} \int_{0}^{1}\left\|\sigma^{-1}(\tilde{u}(r, v)) b(\tilde{u}(r, v))\right\|^{2} d r d v\right) .
\end{array}
$$


Then, by Theorem 6.4, the Cauchy-Schwarz inequality and (6.5),

$$
\begin{aligned}
\mathrm{P}\left\{\mid u_{i}(t, x)-\right. & \left.u_{i}(s, y)|>| z_{1}^{i}-z_{2}^{i} \mid\right\}=\mathrm{E}_{\tilde{\mathrm{P}}}\left[1_{\left\{\left|u^{i}(t, x)-u^{i}(s, y)\right|>\left|z_{1}^{i}-z_{2}^{i}\right|\right\}} L_{t}^{-1}\right] \\
& =\mathrm{E}_{\mathrm{P}}\left[1_{\left\{\left|\tilde{u}^{i}(t, x)-\tilde{u}^{i}(s, y)\right|>\left|z_{1}^{i}-z_{2}^{i}\right|\right\}} J_{t}^{-1}\right] \\
& \leq\left(\mathrm{P}\left\{\left|\tilde{u}^{i}(t, x)-\tilde{u}^{i}(s, y)\right|>\left|z_{1}^{i}-z_{2}^{i}\right|\right\}\right)^{1 / 2}\left(\mathrm{E}_{\mathrm{P}}\left[J_{t}^{-2}\right]\right)^{1 / 2} \\
& \leq 2 \exp \left(-\frac{\left|z_{1}^{i}-z_{2}^{i}\right|^{2}}{C_{T}\left(|t-s|^{1 / 2}+|x-y|\right)}\right)\left(\mathrm{E}_{\mathrm{P}}\left[J_{t}^{-2}\right]\right)^{1 / 2} .
\end{aligned}
$$

Now, hypothesis $\mathbf{P} 1$ and $\mathbf{P 2}$ give

$$
\begin{aligned}
& \mathrm{E}_{\mathrm{P}}\left[J_{t}^{-2}\right] \leq \mathrm{E}_{\mathrm{P}}\left[\operatorname { e x p } \left(\int_{0}^{t} \int_{0}^{1} 2 \sigma^{-1}(\tilde{u}(r, v)) b(\tilde{u}(r, v)) \cdot W(d r, d v)\right.\right. \\
&\left.-\frac{1}{2} \int_{0}^{t} \int_{0}^{1} 4\left\|\sigma^{-1}(\tilde{u}(r, v)) b(\tilde{u}(r, v))\right\|^{2} d r d v\right) \\
&\left.\quad \times \exp \left(\int_{0}^{t} \int_{0}^{1}\left\|\sigma^{-1}(\tilde{u}(r, v)) b(\tilde{u}(r, v))\right\|^{2} d r d v\right)\right] \\
& \leq C,
\end{aligned}
$$

since the second exponential is bounded and the first is an exponential martingale.

Therefore, we have proved that

$$
\mathrm{P}\left\{\left|u_{i}(t, x)-u_{i}(s, y)\right|>\left|z_{1}^{i}-z_{2}^{i}\right|\right\} \leq C \exp \left(-\frac{\left|z_{1}^{i}-z_{2}^{i}\right|^{2}}{C_{T}\left(|t-s|^{1 / 2}+|x-y|\right)}\right)
$$

from which we conclude that

$$
\prod_{i=1}^{d}\left(\mathrm{P}\left\{\left|u_{i}(t, x)-u_{i}(s, y)\right|>\left|z_{1}^{i}-z_{2}^{i}\right|\right\}\right)^{\frac{1}{2 d}} \leq C \exp \left(-\frac{\left\|z_{1}-z_{2}\right\|^{2}}{C_{T}\left(|t-s|^{1 / 2}+|x-y|\right)}\right) .
$$

This proves (6.2).

Proof of (6.3). As in (3.1), using the continuity of the Skorohod integral $\delta$ and Hölder's inequality for Malliavin norms, we obtain

$$
\begin{gathered}
\left\|H_{(1, \ldots, 2 d)}(Z, 1)\right\|_{0,2} \leq C\left\|H_{(1, \ldots, 2 d-1)}(Z, 1)\right\|_{1,4}\left(\sum_{j=1}^{d}\left\|\left(\gamma_{Z}^{-1}\right)_{2 d, j}\right\|_{1,8}\left\|D\left(Z^{j}\right)\right\|_{1,8}\right. \\
\left.+\sum_{j=d+1}^{2 d}\left\|\left(\gamma_{Z}^{-1}\right)_{2 d, j}\right\|_{1,8}\left\|D\left(Z^{j}\right)\right\|_{1,8}\right) .
\end{gathered}
$$


Notice that the entries of $\gamma_{Z}^{-1}$ that appear in this expression belong to sets (3) and (4) of indices, as defined before Theorem 6.3. From Theorem 6.3(a) and Propositions 4.1 and 6.2, we find that this is bounded above by

$C_{T}\left\|H_{(1, \ldots, 2 d-1)}(Z, 1)\right\|_{1,4}\left(\sum_{j=1}^{d}\left(|t-s|^{1 / 2}+|x-y|\right)^{-\frac{1}{2}-d \eta}+\sum_{j=d+1}^{2 d}\left(|t-s|^{1 / 2}+|x-y|\right)^{-1-d \eta+\frac{1}{2}}\right)$,

that is, by

$$
C_{T}\left\|H_{(1, \ldots, 2 d-1)}(Z, 1)\right\|_{1,4}\left(|t-s|^{1 / 2}+|x-y|\right)^{-1 / 2-d \eta} .
$$

Iterating this procedure $d$ times (during which we only encounter coefficients $\left(\gamma_{Z}^{-1}\right)_{m, l}$ for $(m, l)$ in blocs $(3)$ and $(\mathbf{4})$, cf. Theorem $6.3(\mathrm{a}))$, we get, for some integers $m_{0}, k_{0}>0$,

$$
\left\|H_{(1, \ldots, 2 d)}(Z, 1)\right\|_{0,2} \leq C_{T}\left\|H_{(1, \ldots, d)}(Z, 1)\right\|_{m_{0}, k_{0}}\left(|t-s|^{1 / 2}+|x-y|\right)^{-d / 2-d^{2} \eta} .
$$

Again, using the continuity of $\delta$ and Hölder's inequality for the Malliavin norms, we obtain

$$
\begin{array}{r}
\left\|H_{(1, \ldots, d)}(Z, 1)\right\|_{m, k} \leq C\left\|H_{(1, \ldots, d-1)}(Z, 1)\right\|_{m_{1}, k_{1}}\left(\sum_{j=1}^{d}\left\|\left(\gamma_{Z}^{-1}\right)_{d, j}\right\|_{m_{2}, k_{2}}\left\|D\left(Z^{j}\right)\right\|_{m_{3}, k_{3}}\right. \\
\left.+\sum_{j=d+1}^{2 d}\left\|\left(\gamma_{Z}^{-1}\right)_{d, j}\right\|_{m_{4}, k_{4}}\left\|D\left(Z^{j}\right)\right\|_{m_{5}, k_{5}}\right)
\end{array}
$$

for some integers $m_{i}, k_{i}>0, i=1, \ldots, 5$. This time, the entries of $\gamma_{Z}^{-1}$ that appear in this expression come from the sets $(\mathbf{1})$ and $(\mathbf{2})$ of indices. We appeal again to Theorem 6.3(a) and Propositions 4.1 and 6.2 to get

$$
\left\|H_{(1, \ldots, d)}(Z, 1)\right\|_{m, k} \leq C_{T}\left\|H_{(1, \ldots, d-1)}(Z, 1)\right\|_{m_{1}, k_{1}}\left(|t-s|^{1 / 2}+|x-y|\right)^{-d \eta} .
$$

Finally, iterating this procedure $d$ times (during which we encounter coefficients $\left(\gamma_{Z}^{-1}\right)_{m, l}$ for $(m, l)$ in blocs $(\mathbf{1})$ and $(\mathbf{2})$ only, cf. Theorem $6.3(\mathrm{a}))$, and choosing $\eta^{\prime}=4 d^{2} \eta$, we conclude that

$$
\left\|H_{(1, \ldots, 2 d)}(Z, 1)\right\|_{0,2} \leq C_{T}\left(|t-s|^{1 / 2}+|x-y|\right)^{-\left(d+\eta^{\prime}\right) / 2},
$$

which proves (6.3) and concludes the proof of Theorem 1.1(c).

Proof of (6.4). In order to prove (6.4), we proceed exactly along the same lines as in the proof of (6.3) but we appeal to Theorem 6.3(b). This concludes the proof of Theorem $1.1(\mathrm{~d})$.

\subsection{Proof of Theorem 6.3}

Let $Z$ as in (6.1). Since the inverse of the matrix $\gamma_{Z}$ is the inverse of its determinant multiplied by its cofactor matrix, we examine these two factors separately. 
Proposition 6.5. Fix $T>0$ and let $I$ and $J$ be compact intervals as in Theorem 1.1. Assuming $\mathbf{P} 1$, for any $(s, y),(t, x) \in I \times J,(s, y) \neq(t, x), p>1$,

$$
\mathrm{E}\left[\left|\left(A_{Z}\right)_{m, l}\right|^{p}\right]^{1 / p} \leq \begin{cases}c_{p, T}\left(|t-s|^{1 / 2}+|x-y|\right)^{d} & \text { if } \quad(m, l) \in(\mathbf{1}), \\ c_{p, T}\left(|t-s|^{1 / 2}+|x-y|\right)^{d-\frac{1}{2}} & \text { if } \quad(m, l) \in(\mathbf{2}) \text { or }(\mathbf{3}), \\ c_{p, T}\left(|t-s|^{1 / 2}+|x-y|\right)^{d-1} & \text { if } \quad(m, l) \in(\mathbf{4}),\end{cases}
$$

where $A_{Z}$ denotes the cofactor matrix of $\gamma_{Z}$.

Proof. We consider the four different cases.

- If $(m, l) \in(\mathbf{1})$, we claim that

$$
\begin{aligned}
\left|\left(A_{Z}\right)_{m, l}\right| \leq C \sum_{k=0}^{d-1}\{ & \|D(u(s, y))\|_{\mathscr{H}}^{2 k} \times\|D(u(t, x)-u(s, y))\|_{\mathscr{H}}^{2(d-1-k)} \\
& \left.\times\|D(u(s, y))\|_{\mathscr{H}}^{2(d-1-k)} \times\|D(u(t, x)-u(s, y))\|_{\mathscr{H}}^{2(k+1)}\right\} .
\end{aligned}
$$

Indeed, let $A_{Z}^{m, l}=\left(a_{\bar{m}, \bar{l}}^{m, l}\right)_{\bar{m}, \bar{l}=1, \ldots, 2 d-1}$ be the $(2 d-1) \times(2 d-1)$-matrix obtained by removing from $\gamma_{Z}$ its row $m$ and column $l$. Then

$$
\left(A_{Z}\right)_{m, l}=\operatorname{det}\left(A_{Z}^{m, l}\right)=\sum_{\pi \text { permutation of }(1, \ldots, 2 d-1)} \operatorname{sign}(\pi) a_{1, \pi(1)}^{m, l} \cdots a_{2 d-1, \pi(2 d-1)}^{m, l},
$$

where $\operatorname{sign}(\pi) \in\{-1,1\}$ denotes the signature of the permutation $\pi$. Each term of this sum contains one entry from each row and column of $A_{Z}^{m, l}$. If there are $k$ entries taken from bloc (1) of $\gamma_{Z}$, these occupy $k$ rows and columns of $A_{Z}^{m, l}$. Therefore, $d-1-k$ entries must come from the $d-1$ remaining rows of bloc (2), and the same number from the columns of bloc (3). Finally, there remain $k+1$ entries to be taken from bloc (4). Therefore,

$$
\begin{aligned}
& \left|\left(A_{Z}\right)_{m, l}\right| \leq C \sum_{k=0}^{d-1} \sum\{\text { (product of } k \text { entries from }(\mathbf{1})) \\
& \quad \times(\text { product of } d-1-k \text { entries from }(\mathbf{2})) \\
& \quad \times(\text { product of } d-1-k \text { entries from }(\mathbf{3})) \times(\text { product of } k+1 \text { entries from }(\mathbf{4}))\} .
\end{aligned}
$$

Adding all the terms and using the particular form of these terms establishes (6.6).

Regrouping the various factors in (6.6), applying the Cauchy-Schwarz inequality and using (4.1) and Proposition 6.2, we obtain

$$
\begin{aligned}
\mathrm{E}\left[\left|\left(A_{Z}\right)_{m, l}\right|^{p}\right] & \leq C \sum_{k=0}^{d} \mathrm{E}\left[\|D(u(s, y))\|_{\mathscr{H}}^{2(d-1) p} \times\|D(u(t, x)-u(s, y))\|_{\mathscr{H}}^{2 d p}\right] \\
& \leq C_{T}\left(|t-s|^{1 / 2}+|x-y|\right)^{d p} .
\end{aligned}
$$


- If $(m, l) \in(\mathbf{2})$ or $(m, l) \in(\mathbf{3})$, then using the same arguments as above, we obtain

$$
\begin{aligned}
& \quad\left|\left(A_{Z}\right)_{m, l}\right| \\
& \leq C \sum_{k=0}^{d-1} \sum\left\{\|D(u(s, y))\|_{\mathscr{H}}^{2(d-1-k)} \times\|D(u(s, y))\|_{\mathscr{H}}^{k} \times\|D(u(t, x)-u(s, y))\|_{\mathscr{H}}^{k}\right. \\
& \left.\quad \times\|D(u(s, y))\|_{\mathscr{H}}^{k+1} \times\|D(u(t, x)-u(s, y))\|_{\mathscr{\mathscr { H }}}^{k+1} \times\|D(u(t, x)-u(s, y))\|_{\mathscr{H}}^{2(d-1-k)}\right\} \\
& \leq C \sum_{k=0}^{d-1}\left\{\|D(u(s, y))\|_{\mathscr{H}}^{2 d-1} \times\|D(u(t, x)-u(s, y))\|_{\mathscr{H}}^{2 d-1}\right\},
\end{aligned}
$$

from which we conclude, using (4.1) and Proposition 6.2, that

$$
\mathrm{E}\left[\left|\left(A_{Z}\right)_{m, l}\right|^{p}\right] \leq C_{T}\left(|t-s|^{1 / 2}+|x-y|\right)^{\left(d-\frac{1}{2}\right) p} .
$$

- If $(i, j) \in(4)$, we obtain

$$
\begin{aligned}
\left|\left(A_{Z}\right)_{m, l}\right| \leq & C \sum_{k=0}^{d-1} \sum\left\{\|D(u(s, y))\|_{\mathscr{H}}^{2(k+1)} \times\|D(u(s, y))\|_{\mathscr{H}}^{2(d-1-k)}\right. \\
& \left.\times\|D(u(t, x)-D(u(s, y)))\|_{\mathscr{H}}^{2(d-1-k)} \times\|D(u(t, x)-D(u(s, y)))\|_{\mathscr{H}}^{2 k}\right\} \\
\leq & C \sum_{k=0}^{d-1}\left\{\|D(u(s, y))\|_{\mathscr{H}}^{2 d} \times\|D(u(t, x)-u(s, y))\|_{\mathscr{H}}^{2 d-2}\right\},
\end{aligned}
$$

from which we conclude that

$$
\mathrm{E}\left[\left|\left(A_{Z}\right)_{m, l}\right|^{p}\right] \leq C_{T}\left(|t-s|^{1 / 2}+|x-y|\right)^{(d-1) p} .
$$

This concludes the proof of the proposition.

Proposition 6.6. Fix $\eta, T>0$. Assume $\mathbf{P 1}$ and $\mathbf{P 2}$. Let $I$ and $J$ be compact intervals as in Theorem 1.1.

(a) There exists $C$ depending on $T$ and $\eta$ such that for any $(s, y),(t, x) \in I \times J,(s, y) \neq$ $(t, x), p>1$,

$$
\mathrm{E}\left[\left(\operatorname{det} \gamma_{Z}\right)^{-p}\right]^{1 / p} \leq C\left(|t-s|^{1 / 2}+|x-y|\right)^{-d(1+\eta)} .
$$

(b) There exists $C$ only depending on $T$ such that for any $s=t \in I, x, y \in J, x \neq y$, $p>1$,

$$
\mathrm{E}\left[\left(\operatorname{det} \gamma_{Z}\right)^{-p}\right]^{1 / p} \leq C(|x-y|)^{-d}
$$

Assuming this proposition, we will be able to conclude the proof of Theorem 6.3, after establishing the following estimate on the derivative of the Malliavin matrix. 
Proposition 6.7. Fix $T>0$. Let $I$ and $J$ be compact intervals as in Theorem 1.1. Assuming $\mathbf{P} 1$, for any $(s, y),(t, x) \in I \times J,(s, y) \neq(t, x), p>1$ and $k \geq 1$,

$$
\mathrm{E}\left[\left\|D^{k}\left(\gamma_{Z}\right)_{m, l}\right\|_{\mathscr{H} \otimes k}^{p}\right]^{1 / p} \leq \begin{cases}c_{k, p, T} & \text { if } \quad(m, l) \in(\mathbf{1}), \\ c_{k, p, T}\left(|t-s|^{1 / 2}+|x-y|\right)^{1 / 2} & \text { if } \quad(m, l) \in(\mathbf{2}) \text { or }(\mathbf{3}), \\ c_{k, p, T}\left(|t-s|^{1 / 2}+|x-y|\right) & \text { if } \quad(m, l) \in(\mathbf{4}) .\end{cases}
$$

Proof. We consider the four different blocs.

- If $(m, l) \in(4)$, proceeding as in DAlang AND NuAlart [DN04, p.2131] and appealing to Proposition 6.2 twice, we obtain

$$
\begin{aligned}
& \mathrm{E}\left[\left\|D^{k}\left(\gamma_{Z}\right)_{m, l}\right\|_{\mathscr{H} \otimes k}^{p}\right] \\
& =\mathrm{E}\left[\left\|D^{k}\left(\int_{0}^{T} d r \int_{0}^{1} d v D_{r, v}\left(u_{m}(t, x)-u_{m}(s, y)\right) \cdot D_{r, v}\left(u_{l}(t, x)-u_{l}(s, y)\right)\right)\right\|_{\mathscr{H} \otimes k}^{p}\right] \\
& \leq(k+1)^{p-1} \sum_{j=0}^{k}\left(\begin{array}{l}
k \\
j
\end{array}\right)^{p} \mathrm{E}\left[\| \int_{0}^{T} d r \int_{0}^{1} d v D^{j} D_{r, v}\left(u_{m}(t, x)-u_{m}(s, y)\right)\right. \\
& \left.\cdot D^{k-j} D_{r, v}\left(u_{l}(t, x)-u_{l}(s, y)\right) \|_{\mathscr{H} \otimes k}^{p}\right] \\
& \leq \tilde{C}_{T}(k+1)^{p-1} \sum_{j=0}^{k}\left(\begin{array}{c}
k \\
j
\end{array}\right)^{p}\left\{\left(E\left[\left\|D^{j} D\left(u_{m}(t, x)-u_{m}(s, y)\right)\right\|_{\mathscr{H} \otimes(j+1)}^{2 p}\right]\right)^{1 / 2}\right. \\
& \left.\times\left(\mathrm{E}\left[\left\|D^{k-j} D\left(u_{l}(t, x)-u_{l}(s, y)\right)\right\|_{\mathscr{H} \otimes(k-j+1)}^{2 p}\right]\right)^{1 / 2}\right\} \\
& \leq C_{T}\left(|t-s|^{1 / 2}+|x-y|\right)^{p}
\end{aligned}
$$

- If $(m, l) \in(\mathbf{2})$ or $(m, l) \in(\mathbf{3})$, proceeding as above and appealing to (4.1) and Proposition 6.2 , we get

$$
\begin{aligned}
& \mathrm{E}\left[\left\|D^{k}\left(\gamma_{Z}\right)_{m, l}\right\|_{\mathscr{H} \otimes k}^{p}\right] \\
& \begin{aligned}
\leq \tilde{C}_{T}(k+1)^{p-1} \sum_{j=0}^{k}\left(\begin{array}{c}
k \\
j
\end{array}\right)^{p}\left\{\left(\mathrm{E}\left[\left\|D^{j} D\left(u_{m}(t, x)-u_{m}(s, y)\right)\right\|_{\mathscr{H} \otimes(j+1)}^{2 p}\right]\right)^{1 / 2}\right. \\
\left.\times\left(\mathrm{E}\left[\left\|D^{k-j} D\left(u_{l}(s, y)\right)\right\|_{\mathscr{H} \otimes(k-j+1)}^{2 p}\right]\right)^{1 / 2}\right\} \\
\leq C_{T}\left(|t-s|^{1 / 2}+|x-y|\right)^{p / 2} .
\end{aligned}
\end{aligned}
$$

- If $(m, l) \in(\mathbf{1})$, using (4.1), we obtain

$$
\begin{aligned}
& \mathrm{E}\left[\left\|D^{k}\left(\gamma_{Z}\right)_{m, l}\right\|_{\mathscr{H} \otimes k}^{p}\right] \\
& \begin{aligned}
\leq \tilde{C}_{T}(k+1)^{p-1} \sum_{j=0}^{k}\left(\begin{array}{c}
k \\
j
\end{array}\right)^{p} & \left\{\left(\mathrm{E}\left[\left\|D^{j} D\left(u_{m}(s, y)\right)\right\|_{\mathscr{H} \otimes(j+1)}^{2 p}\right]\right)^{1 / 2}\right. \\
& \left.\times\left(\mathrm{E}\left[\left\|D^{k-j} D\left(u_{l}(s, y)\right)\right\|_{\mathscr{H} \otimes(k-j+1)}^{2 p}\right]\right)^{1 / 2}\right\} \\
\leq C_{T} . &
\end{aligned}
\end{aligned}
$$


Proof of Theorem 6.3. When $k=0$, the result follows directly using the fact that the inverse of a matrix is the inverse of its determinant multiplied by its cofactor matrix and the estimates of Propositions 6.5 and 6.6.

For $k \geq 1$, we shall establish the following two properties.

(a) For any $(s, y),(t, x) \in I \times J,(s, y) \neq(t, x), s \leq t, k \geq 1$ and $p>1$,

$$
\mathrm{E}\left[\left\|D^{k}\left(\gamma_{Z}^{-1}\right)_{m, l}\right\|_{\mathscr{H} \otimes k}^{p}\right]^{1 / p} \leq \begin{cases}c_{k, p, \eta, T}\left(|t-s|^{1 / 2}+|x-y|\right)^{-d \eta} & \text { if }(m, l) \in(\mathbf{1}), \\ c_{k, p, \eta, T}\left(|t-s|^{1 / 2}+|x-y|\right)^{-1 / 2-d \eta} & \text { if }(m, l) \in(\mathbf{2}),(\mathbf{3}), \\ c_{k, p, \eta, T}\left(|t-s|^{1 / 2}+|x-y|\right)^{-1-d \eta} & \text { if }(m, l) \in(\mathbf{4}) .\end{cases}
$$

(b) For any $s=t \in I, x, y \in J, x \neq y, k \geq 1$ and $p>1$,

$$
\mathrm{E}\left[\left\|D^{k}\left(\gamma_{Z}^{-1}\right)_{m, l}\right\|_{\mathscr{H} \otimes k}^{p}\right]^{1 / p} \leq\left\{\begin{array}{lll}
c_{k, p, T} & \text { if } \quad(m, l) \in(\mathbf{1}) \\
c_{k, p, T}|x-y|^{-1 / 2} & \text { if } \quad(m, l) \in(\mathbf{2}) \text { or }(\mathbf{3}), \\
c_{k, p, T}|x-y|^{-1} & \text { if } \quad(m, l) \in(\mathbf{4}) .
\end{array}\right.
$$

Since

$$
\left\|\left(\gamma_{Z}^{-1}\right)_{m, l}\right\|_{k, p}=\left\{\mathrm{E}\left[\left|\left(\gamma_{Z}^{-1}\right)_{m, l}\right|^{p}\right]+\sum_{j=1}^{k} \mathrm{E}\left[\left\|D^{j}\left(\gamma_{Z}^{-1}\right)_{m, l}\right\|_{\mathscr{H} \otimes j}^{p}\right]\right\}^{1 / p}
$$

(a) and (b) prove the theorem.

We now prove (a) and (b). When $k=1$, we will use (3.3) written as a matrix product:

$$
D\left(\gamma_{Z}^{-1}\right)=\gamma_{Z}^{-1} D\left(\gamma_{Z}\right) \gamma_{Z}^{-1} .
$$

Writing (6.8) in bloc product matrix notation with blocs (1), (2), (3) and (4), we get that

$$
\begin{aligned}
D\left(\left(\gamma_{Z}^{-1}\right)^{(1)}\right)=( & \left.\gamma_{Z}^{-1}\right)^{(1)} D\left(\gamma_{Z}^{(1)}\right)\left(\gamma_{Z}^{-1}\right)^{(1)}+\left(\gamma_{Z}^{-1}\right)^{(1)} D\left(\gamma_{Z}^{(2)}\right)\left(\gamma_{Z}^{-1}\right)^{(3)} \\
& +\left(\gamma_{Z}^{-1}\right)^{(2)} D\left(\gamma_{Z}^{(3)}\right)\left(\gamma_{Z}^{-1}\right)^{(1)}+\left(\gamma_{Z}^{-1}\right)^{(2)} D\left(\gamma_{Z}^{(4)}\right)\left(\gamma_{Z}^{-1}\right)^{(3)}, \\
D\left(\left(\gamma_{Z}^{-1}\right)^{(2)}\right)=\left(\gamma_{Z}^{-1}\right)^{(1)} D\left(\gamma_{Z}^{(1)}\right)\left(\gamma_{Z}^{-1}\right)^{(2)}+\left(\gamma_{Z}^{-1}\right)^{(1)} D\left(\gamma_{Z}^{(2)}\right)\left(\gamma_{Z}^{-1}\right)^{(4)} & \\
& +\left(\gamma_{Z}^{-1}\right)^{(2)} D\left(\gamma_{Z}^{(3)}\right)\left(\gamma_{Z}^{-1}\right)^{(2)}+\left(\gamma_{Z}^{-1}\right)^{(2)} D\left(\gamma_{Z}^{(4)}\right)\left(\gamma_{Z}^{-1}\right)^{(4)}, \\
D\left(\left(\gamma_{Z}^{-1}\right)^{(3)}\right)=\left(\gamma_{Z}^{-1}\right)^{(3)} D\left(\gamma_{Z}^{(1)}\right)\left(\gamma_{Z}^{-1}\right)^{(1)}+\left(\gamma_{Z}^{-1}\right)^{(3)} D\left(\gamma_{Z}^{(2)}\right)\left(\gamma_{Z}^{-1}\right)^{(3)} & \\
& +\left(\gamma_{Z}^{-1}\right)^{(4)} D\left(\gamma_{Z}^{(3)}\right)\left(\gamma_{Z}^{-1}\right)^{(1)}+\left(\gamma_{Z}^{-1}\right)^{(4)} D\left(\gamma_{Z}^{(4)}\right)\left(\gamma_{Z}^{-1}\right)^{(3)}, \\
D\left(\left(\gamma_{Z}^{-1}\right)^{(4)}\right)=\left(\gamma_{Z}^{-1}\right)^{(3)} D\left(\gamma_{Z}^{(1)}\right)\left(\gamma_{Z}^{-1}\right)^{(2)}+\left(\gamma_{Z}^{-1}\right)^{(3)} D\left(\gamma_{Z}^{(2)}\right)\left(\gamma_{Z}^{-1}\right)^{(4)} & \\
& +\left(\gamma_{Z}^{-1}\right)^{(4)} D\left(\gamma_{Z}^{(3)}\right)\left(\gamma_{Z}^{-1}\right)^{(2)}+\left(\gamma_{Z}^{-1}\right)^{(4)} D\left(\gamma_{Z}^{(4)}\right)\left(\gamma_{Z}^{-1}\right)^{(4)} .
\end{aligned}
$$

It now suffices to apply Hölder's inequality to each block and use the estimates of the case $k=0$ and Proposition 6.7 to obtain the desired result for $k=1$. For instance, for 
$(m, l) \in(\mathbf{1})$

$$
\begin{aligned}
& \mathrm{E}\left[\left\|\left(\left(\gamma_{Z}^{-1}\right)^{(2)} D\left(\gamma_{Z}^{(4)}\right)\left(\gamma_{Z}^{-1}\right)^{(3)}\right)_{m, l}\right\|_{\mathscr{H}}^{p}\right]^{1 / p} \\
& \leq \sup _{m_{1}, l_{1}} \mathrm{E}\left[\left|\left(\left(\gamma_{Z}^{-1}\right)^{(2)}\right)_{m_{1}, l_{1}}\right|^{2 p}\right]^{1 /(2 p)} \sup _{m_{2}, l_{2}} \mathrm{E}\left[\left\|\left(D\left(\gamma_{Z}^{(4)}\right)\right)_{m_{2}, l_{2}}\right\|_{\mathscr{H}}^{4 p}\right]^{1 /(4 p)} \\
& \quad \times \sup _{m_{3}, l_{3}} \mathrm{E}\left[\left|\left(\left(\gamma_{Z}^{-1}\right)^{(3)}\right)_{m_{3}, l_{3}}\right|^{4 p}\right]^{1 /(4 p)} \\
& \leq c\left(|t-s|^{1 / 2}+|x-y|\right)^{-\frac{1}{2}-d \eta+1-\frac{1}{2}-d \eta}=c\left(|t-s|^{1 / 2}+|x-y|\right)^{-2 d \eta} .
\end{aligned}
$$

For $k \geq 1$, in order to calculate $D^{k+1}\left(\gamma_{Z}^{(\cdot)}\right)$, we will need to compute $D^{k}\left(\gamma_{Z}^{-1} D\left(\gamma_{Z}\right) \gamma_{Z}^{-1}\right)$. For bloc numbers $i_{1}, i_{2}, i_{3} \in\{1,2,3,4\}$ and $k \geq 1$, we have

$$
\begin{aligned}
& D^{k}\left(\left(\gamma_{Z}^{-1}\right)^{\left(i_{1}\right)} D\left(\gamma_{Z}^{\left(i_{2}\right)}\right)\left(\gamma_{Z}^{-1}\right)^{\left(i_{3}\right)}\right) \\
& =\sum_{\substack{j_{1}+j_{2}+j_{3}=k \\
j_{i} \in\{0, \ldots, k\}}}\left(\begin{array}{c}
k \\
j_{1} j_{2} j_{3}
\end{array}\right) D^{j_{1}}\left(\left(\gamma_{Z}^{-1}\right)^{\left(i_{1}\right)}\right) D^{j_{2}}\left(D\left(\gamma_{Z}^{\left(i_{2}\right)}\right)\right) D^{j_{3}}\left(\left(\gamma_{Z}^{-1}\right)^{\left(i_{3}\right)}\right) .
\end{aligned}
$$

Note that by Proposition 6.7, the norms of the derivatives $D^{j_{2}}\left(D\left(\gamma_{Z}^{\left(i_{2}\right)}\right)\right.$ of $\gamma_{Z}^{\left(i_{2}\right)}$ are of the same order for all $j_{2}$. Hence, we appeal again to Hölder's inequality and Proposition 6.7, and use a recursive argument in order to obtain the desired bounds.

Proof of Proposition 6.6. The main idea for the proof of Proposition 6.6 is to use a perturbation argument. Indeed, for $(t, x)$ close to $(s, y)$, the matrix $\gamma_{Z}$ is close to

$$
\hat{\gamma}=\left(\begin{array}{ccc}
\gamma_{Z}^{(1)} & \vdots & 0 \\
\cdots & \vdots & \cdots \\
0 & \vdots & 0
\end{array}\right) .
$$

The matrix $\hat{\gamma}$ has $d$ eigenvectors of the form $\left(\hat{\lambda}^{1}, \mathbf{0}\right), \ldots,\left(\hat{\lambda}^{d}, \mathbf{0}\right)$, where $\hat{\lambda}^{1}, \ldots, \hat{\lambda}^{d} \in \mathbb{R}^{d}$ are eigenvectors of $\gamma_{Z}^{(1)}=\gamma_{u(s, y)}$, and $\mathbf{0}=(0, \ldots, 0) \in \mathbb{R}^{d}$, and $d$ other eigenvectors of the form $\left(\mathbf{0}, e^{i}\right)$ where $e^{1}, \ldots, e^{d}$ is a basis of $\mathbb{R}^{d}$. These last eigenvectors of $\hat{\gamma}$ are associated with the eigenvalue 0 , while the former are associated with eigenvalues of order 1 , as can be seen in the proof of Proposition 4.2.

We now write

$$
\operatorname{det} \gamma_{Z}=\prod_{i=1}^{2 d}\left(\xi^{i}\right)^{T} \gamma_{Z} \xi^{i},
$$

where $\xi=\left\{\xi^{1}, \ldots, \xi^{2 d}\right\}$ is an orthonormal basis of $\mathbb{R}^{2 d}$ consisting of eigenvectors of $\gamma_{Z}$. We then expect that for $(t, x)$ close to $(s, y)$, there will be $d$ eigenvectors close to the subspace 
generated by the $\left(\hat{\lambda}^{i}, \mathbf{0}\right)$, which will contribute a factor of order 1 to the product in $(6.9)$, and $d$ other eigenvectors, close to the subspace generated by the $\left(\mathbf{0}, e^{i}\right)$, that will each contribute a factor of order $\left(|t-s|^{1 / 2}+|x-y|\right)^{-1-\eta}$ to the product. Note that if we do not distinguish between these two types of eigenvectors, but simply bound below the product by the smallest eigenvalue to the power $2 d$, following the approach used in the proof of Proposition 4.2, then we would obtain $C\left(|t-s|^{1 / 2}+|x-y|\right)^{-2 d p}$ in the right-hand side of (6.7), which would not be the correct order.

We now carry out this somewhat involved perturbation argument. Consider the spaces $E_{1}=\left\{(\lambda, \mathbf{0}): \lambda \in \mathbb{R}^{d}, \mathbf{0} \in \mathbb{R}^{d}\right\}$ and $E_{2}=\left\{(\mathbf{0}, \mu): \mu \in \mathbb{R}^{d}, \mathbf{0} \in \mathbb{R}^{d}\right\}$. Note that every $\xi^{i}$ can be written as

$$
\xi^{i}=\left(\lambda^{i}, \mu^{i}\right)=\alpha_{i}\left(\tilde{\lambda}^{i}, \mathbf{0}\right)+\sqrt{1-\alpha_{i}^{2}}\left(\mathbf{0}, \tilde{\mu}^{i}\right),
$$

where $\lambda^{i}, \mu^{i} \in \mathbb{R}^{d},\left(\tilde{\lambda}^{i}, \mathbf{0}\right) \in E_{1},\left(\mathbf{0}, \tilde{\mu}^{i}\right) \in E_{2}$, with $\left\|\tilde{\lambda}^{i}\right\|=\left\|\tilde{\mu}^{i}\right\|=1$ and $0 \leq \alpha_{i} \leq 1$. Note in particular that $\left\|\xi^{i}\right\|^{2}=\left\|\lambda^{i}\right\|^{2}+\left\|\mu^{i}\right\|^{2}=1$ (norms of elements of $\mathbb{R}^{d}$ or $\mathbb{R}^{2 d}$ are Euclidean norms).

Lemma 6.8. Given a sufficiently small $\alpha_{0}>0$, with probability one, there exist at least $d$ of these vectors, say $\xi^{i_{1}}, \ldots, \xi^{i_{d}}$, such that $\alpha_{i_{1}} \geq \alpha_{0}, \ldots, \alpha_{i_{d}} \geq \alpha_{0}$.

Proof. Observe that as $\xi$ is an orthogonal family and for $i \neq j$, the Euclidean inner product of $\xi^{i}$ and $\xi^{j}$ is

$$
\xi^{i} \cdot \xi^{j}=\alpha_{i} \alpha_{j}\left(\tilde{\lambda}^{i} \cdot \tilde{\lambda}^{j}\right)+\sqrt{1-\alpha_{i}^{2}} \sqrt{1-\alpha_{j}^{2}}\left(\tilde{\mu}^{i} \cdot \tilde{\mu}^{j}\right)=0 .
$$

For $\alpha_{0}>0$, let $D=\left\{i \in\{1, \ldots, 2 d\}: \alpha_{i}<\alpha_{0}\right\}$. Then, for $i, j \in D, i \neq j$, if $\alpha_{0}<\frac{1}{2}$, then

$$
\left|\tilde{\mu}^{i} \cdot \tilde{\mu}^{j}\right|=\frac{\alpha_{i} \alpha_{j}}{\sqrt{1-\alpha_{i}^{2}} \sqrt{1-\alpha_{j}^{2}}}\left|\tilde{\lambda}^{i} \cdot \tilde{\lambda}^{j}\right| \leq \frac{\alpha_{0}^{2}}{1-\alpha_{0}^{2}}\left\|\tilde{\lambda}^{i}\right\|\left\|\tilde{\lambda}^{j}\right\| \leq \frac{1}{3} \alpha_{0}^{2} .
$$

Since the diagonal terms of the matrix $\left(\tilde{\mu}^{i} \cdot \tilde{\mu}^{j}\right)_{i, j \in D}$ are all equal to 1 , for $\alpha_{0}$ sufficiently small, it follows that $\operatorname{det}\left(\left(\tilde{\mu}^{i} \cdot \tilde{\mu}^{j}\right)_{i, j \in D}\right) \neq 0$. Therefore, $\left\{\tilde{\mu}^{i}, i \in D\right\}$ is a linearly independent family, and, as $\left(\mathbf{0}, \tilde{\mu}^{i}\right) \in E_{2}$, for $i=1, \ldots, 2 d$, we conclude that a.s., $\operatorname{card}(D) \leq \operatorname{dim}\left(E_{2}\right)=d$. Therefore, there exists a set of indexes $\left\{i_{1}, \ldots, i_{d}\right\} \subset D^{c}$ and so $\alpha_{i_{1}} \geq \alpha_{0}, \ldots, \alpha_{i_{d}} \geq \alpha_{0}$.

By Lemma 6.8 and Cauchy-Schwarz inequality, one can write

$$
\begin{aligned}
\mathrm{E}\left[\left(\operatorname{det} \gamma_{Z}\right)^{-p}\right]^{1 / p} \leq \sum_{K \subset\{1, \ldots, 2 d\},|K|=d}\left(\mathrm{E}\left[\mathbf{1}_{A_{K}}\left(\prod_{i \in K}\left(\xi^{i}\right)^{T} \gamma_{Z} \xi^{i}\right)^{-2 p}\right]\right)^{1 /(2 p)} \\
\times\left(\mathrm{E}\left[\left(\inf _{\substack{\xi=(\lambda, \mu) \in \mathbb{R}^{2 d}: \\
\|\lambda\|^{2}+\|\mu\|^{2}=1}} \xi^{\top} \gamma_{Z} \xi\right)^{-2 d p}\right]\right)^{1 /(2 p)}
\end{aligned}
$$

where $A_{K}=\cap_{i \in K}\left\{\alpha_{i} \geq \alpha_{0}\right\}$.

With this, Propositions 6.9 and 6.13 below conclude the proof of Proposition 6.6. 


\subsubsection{Small Eigenvalues}

Let $I$ and $J$ two compact intervals as in Theorem 1.1.

Proposition 6.9. Fix $\eta, T>0$. Assume $\mathbf{P 1}$ and $\mathbf{P 2}$.

(a) There exists $C$ depending on $\eta$ and $T$ such that for all $s, t \in I, 0 \leq t-s<1, x, y \in J$, $(s, y) \neq(t, x)$, and $p>1$,

$$
\mathrm{E}\left[\left(\inf _{\substack{\xi=(\lambda, \mu) \in \mathbb{R}^{2 d:} \\\|\lambda\|^{2}+\|\mu\|^{2}=1}} \xi^{\top} \gamma_{Z} \xi\right)^{-2 d p}\right] \leq C\left(|t-s|^{1 / 2}+|x-y|\right)^{-2 d p(1+\eta)} .
$$

(b) There exists $C$ depending only on $T$ such that for all $s=t \in I, x, y \in J, x \neq y$, and $p>1$,

$$
\mathrm{E}\left[\left(\inf _{\substack{\xi=(\lambda, \mu) \in \mathbb{R}^{2 d}: \\\|\lambda\|^{2}+\|\mu\|^{2}=1}} \xi^{\top} \gamma_{Z} \xi\right)^{-2 d p}\right] \leq C(|x-y|)^{-2 d p}
$$

Proof. We begin by proving (a). Since $\gamma_{Z}$ is a matrix of inner products, we can write

$$
\xi^{\top} \gamma_{Z} \xi=\sum_{k=1}^{d} \int_{0}^{T} d r \int_{0}^{1} d v\left(\sum_{i=1}^{d}\left(\lambda_{i} D_{r, v}^{(k)}\left(u_{i}(s, y)\right)+\mu_{i}\left(D_{r, v}^{(k)}\left(u_{i}(t, x)\right)-D_{r, v}^{(k)}\left(u_{i}(s, y)\right)\right)\right)\right)^{2} .
$$

From here on, the proof is divided into two cases.

Case 1. In the first case, we assume that $t-s>0$ and $|x-y|^{2} \leq t-s$. Choose and fix an $\epsilon \in\left(0,(t-s) \wedge\left(\frac{1}{4}\right)^{2 / \eta}\right)$. Then we may write

$$
\xi^{\top} \gamma_{Z} \xi \geq J_{1}+J_{2}
$$

where

$$
\begin{aligned}
J_{1} & :=\sum_{k=1}^{d} \int_{s-\epsilon}^{s} d r \int_{0}^{1} d v\left(\sum_{i=1}^{d}\left(\lambda_{i}-\mu_{i}\right)\left[G_{s-r}(y, v) \sigma_{i k}(u(r, v))+a_{i}(k, r, v, s, y)\right]+W\right)^{2}, \\
J_{2} & :=\sum_{k=1}^{d} \int_{t-\epsilon}^{t} d r \int_{0}^{1} d v W^{2}
\end{aligned}
$$

$a_{i}(k, r, v, s, y)$ is defined in (4.3) and

$$
W:=\sum_{i=1}^{d}\left[\mu_{i} G_{t-r}(x, v) \sigma_{i k}(u(r, v))+\mu_{i} a_{i}(k, r, v, t, x)\right] .
$$

Therefore,

$$
\inf _{\|\xi\|=1} \xi^{\top} \gamma_{Z} \xi \geq \min \left(\inf _{\|\xi\|=1,\|\mu\| \geq \epsilon^{\eta / 2}} J_{2}, \inf _{\|\xi\|=1,\|\mu\| \leq \epsilon^{\eta / 2}} J_{1}\right)
$$


We are going to prove that

$$
\begin{aligned}
& \inf _{\|\xi\|=1,\|\mu\| \geq \epsilon^{\eta / 2}} J_{2} \geq \epsilon^{\frac{1}{2}+\eta}-Y_{1, \epsilon}, \\
& \inf _{\|\xi\|=1,\|\mu\| \leq \epsilon^{\eta / 2}} J_{1} \geq \epsilon^{1 / 2}-Y_{2, \epsilon},
\end{aligned}
$$

where, for all $q \geq 1$,

$$
\mathrm{E}\left[\left|Y_{1, \epsilon}\right|^{q}\right] \leq c_{1}(q) \epsilon^{q} \quad \text { and } \quad \mathrm{E}\left[\left|Y_{2, \epsilon}\right|^{q}\right] \leq c_{2}(q) \epsilon^{q\left(\frac{1}{2}+\eta\right)} .
$$

We assume these, for the time being, and finish the proof of the proposition in Case 1 . Then we will return to proving (6.12) and (6.13).

We can combine (6.12) and (6.13) with Proposition 3.5 to find that

$$
\begin{aligned}
\mathrm{E}\left[\left(\inf _{\|\xi\|=1} \xi^{\top} \gamma_{Z} \xi\right)^{-2 p d}\right] & \leq c\left((t-s) \wedge\left(\frac{1}{4}\right)^{2 / \eta}\right)^{-2 p d\left(\frac{1}{2}+\eta\right)} \leq c^{\prime}(t-s)^{-2 p d\left(\frac{1}{2}+\eta\right)} \\
& \leq \tilde{c}\left[(t-s)^{1 / 2}+|x-y|\right]^{-2 p d(1+2 \eta)}
\end{aligned}
$$

whence follows the proposition in the case that $|x-y|^{2} \leq t-s<1$. Now we complete our proof of Case 1 by deriving (6.12) and (6.13).

Let us begin with the term that involves $J_{2}$. Inequality (4.4) implies that

$$
\inf _{\|\xi\|=1,\|\mu\| \geq \epsilon^{\eta / 2}} J_{2} \geq \hat{Y}_{1, \epsilon}-Y_{1, \epsilon}
$$

where

$$
\begin{aligned}
\hat{Y}_{1, \epsilon} & :=\frac{2}{3} \inf _{\|\mu\| \geq \epsilon^{\eta / 2}} \sum_{k=1}^{d} \int_{t-\epsilon}^{t} d r \int_{0}^{1} d v\left(\sum_{i=1}^{d} \mu_{i} \sigma_{i k}(u(r, v))\right)^{2} G_{t-r}^{2}(x, v), \\
Y_{1, \epsilon} & :=2 \sup _{\|\mu\| \geq \epsilon^{\eta / 2}} \sum_{k=1}^{d} \int_{t-\epsilon}^{t} d r \int_{0}^{1} d v\left(\sum_{i=1}^{d} \mu_{i} a_{i}(k, r, v, t, x)\right)^{2} .
\end{aligned}
$$

In agreement with hypothesis P2, and thanks to Lemma 7.2,

$$
\hat{Y}_{1, \epsilon} \geq c \inf _{\|\mu\| \geq \epsilon^{\eta / 2}}\|\mu\|^{2} \epsilon^{1 / 2} \geq c \epsilon^{\frac{1}{2}+\eta} .
$$

Next we apply Lemma 6.11 below [with $s:=t]$ to find that $\mathrm{E}\left[\left|Y_{1, \epsilon}\right|^{q}\right] \leq c \epsilon^{q}$. This proves the bounds in (6.12) and (6.13) that concern $J_{2}$ and $Y_{1, \epsilon}$.

In order to derive the second bound in (6.12), we appeal to (4.4) once more to find that

$$
\inf _{\|\xi\|=1,\|\mu\| \leq \epsilon^{\eta / 2}} J_{1} \geq \hat{Y}_{2, \epsilon}-Y_{2, \epsilon}
$$

where

$$
\hat{Y}_{2, \epsilon}:=\frac{2}{3} \inf _{\|\mu\| \leq \epsilon^{\eta / 2}} \sum_{k=1}^{d} \int_{s-\epsilon}^{s} d r \int_{0}^{1} d v\left(\sum_{i=1}^{d}\left(\lambda_{i}-\mu_{i}\right) \sigma_{i k}(u(r, v))\right)^{2} G_{s-r}^{2}(y, v),
$$


and

$$
Y_{2, \epsilon}:=6\left(W_{1}+W_{2}+W_{3}\right)
$$

where

$$
\begin{aligned}
& W_{1}:=\sup _{\|\mu\| \leq \epsilon^{\eta / 2}} \sum_{k=1}^{d} \int_{s-\epsilon}^{s} d r \int_{0}^{1} d v\left(\sum_{i=1}^{d} \mu_{i} G_{t-r}(x, v) \sigma_{i k}(u(r, v))\right)^{2}, \\
& W_{2}:=\sup _{\|\xi\|=1} \sum_{k=1}^{d} \int_{s-\epsilon}^{s} d r \int_{0}^{1} d v\left(\sum_{i=1}^{d}\left(\lambda_{i}-\mu_{i}\right) a_{i}(k, r, v, s, y)\right)^{2}, \\
& W_{3}:=\sup _{\|\mu\| \leq \epsilon^{\eta / 2}} \sum_{k=1}^{d} \int_{s-\epsilon}^{s} d r \int_{0}^{1} d v\left(\sum_{i=1}^{d} \mu_{i} a_{i}(k, r, v, t, x)\right)^{2} .
\end{aligned}
$$

Hypothesis $\mathbf{P} 2$ and Lemma 7.2 together imply that $\hat{Y}_{2, \epsilon} \geq c\|\lambda-\mu\| \epsilon^{1 / 2}$. Therefore, because $\|\lambda\|^{2}+\|\mu\|^{2}=1$ and $\|\mu\| \leq \epsilon^{\eta / 2} \leq \frac{1}{4}$

$$
\inf _{\|\xi\|=1,\|\mu\| \leq \epsilon^{\eta / 2}} J_{1} \geq \tilde{c} \epsilon^{1 / 2}-6\left(W_{1}+W_{2}+W_{3}\right) .
$$

Next, we apply the Cauchy-Schwarz inequality to find that

$$
\begin{aligned}
\mathrm{E}\left[\left|W_{1}\right|^{q}\right] & \leq \sup _{\|\mu\| \leq \epsilon^{\eta / 2}}\|\mu\|^{2 q} \times \mathrm{E}\left[\left|\sum_{k=1}^{d} \int_{s-\epsilon}^{s} d r \int_{0}^{1} d v \sum_{i=1}^{d}\left(\sigma_{i k}(u(r, v))\right)^{2} G_{t-r}^{2}(x, v)\right|^{q}\right] \\
& \leq c \epsilon^{q \eta}\left|\sum_{k=1}^{d} \int_{s-\epsilon}^{s} d r \int_{0}^{1} d v G_{t-r}^{2}(x, v)\right|^{q},
\end{aligned}
$$

thanks to hypothesis P1. In light of this and using the change of variables $r^{\prime}=t-r$, Lemma 7.4 implies that $\mathrm{E}\left[\left|W_{1}\right|^{q}\right] \leq c \epsilon^{q\left(\frac{1}{2}+\eta\right)}$.

In order to bound the $q$-th moment of $\left|W_{2}\right|$, we use the Cauchy-Schwarz inequality together with hypothesis $\mathbf{P 1}$, and write

$$
\begin{aligned}
\mathrm{E}\left[\left|W_{2}\right|^{q}\right] & \leq \sup _{\|\mu\| \leq \epsilon^{\eta / 2}}\|\lambda-\mu\|^{2 q} \times \mathrm{E}\left[\left|\sum_{k=1}^{d} \int_{s-\epsilon}^{s} d r \int_{0}^{1} d v \sum_{i=1}^{d} a_{i}^{2}(k, r, v, s, y)\right|^{q}\right] \\
& \leq C \mathrm{E}\left[\left|\sum_{k=1}^{d} \int_{s-\epsilon}^{s} d r \int_{0}^{1} d v \sum_{i=1}^{d} a_{i}^{2}(k, r, v, s, y)\right|^{q}\right] .
\end{aligned}
$$

We apply Lemma 6.11 below [with $s:=t]$ to find that $\mathrm{E}\left[\left|W_{2}\right|^{q}\right] \leq c \epsilon^{q}$.

Similarly, we find using Lemma 6.11 that

$$
\begin{aligned}
\mathrm{E}\left[\left|W_{3}\right|^{q}\right] & \leq \sup _{\|\mu\| \leq \epsilon^{\eta / 2}}\|\mu\|^{2 q} \times \mathrm{E}\left[\left|\sum_{k=1}^{d} \int_{s-\epsilon}^{s} d r \int_{0}^{1} d v \sum_{i=1}^{d} a_{i}^{2}(k, r, v, t, x)\right|^{q}\right] \\
& \leq c \epsilon^{q \eta}(t-s+\epsilon)^{q / 2} \epsilon^{q / 2} \\
& \leq c \epsilon^{q\left(\frac{1}{2}+\eta\right)} .
\end{aligned}
$$


The preceding bounds for $W_{1}, W_{2}$, and $W_{3}$ prove, in conjunction, that $\mathrm{E}\left[\left|Y_{2, \epsilon}\right|^{q}\right] \leq$ $c_{2}(q) \epsilon^{q\left(\frac{1}{2}+\eta\right)}$. This and (6.14) together prove the bounds in (6.12) and (6.13) that concern $J_{1}$ and $Y_{2, \epsilon}$, whence follows the result in Case 1.

Case 2. Now we work on the second case where $|x-y|>0$ and $|x-y|^{2} \geq t-s \geq 0$. Let $\epsilon>0$ be such that $(1+\alpha) \epsilon^{1 / 2}<\frac{1}{2}|x-y|$, where $\alpha>0$ is large but fixed; its specific value will be decided on later (just before (6.27)). Then

$$
\xi^{\top} \gamma_{Z} \xi \geq I_{1}+I_{2}
$$

where

$$
\begin{aligned}
I_{1} & :=\sum_{k=1}^{d} \int_{s-\epsilon}^{s} d r \int_{0}^{1} d v\left(\mathscr{S}_{1}+\mathscr{S}_{2}\right)^{2}, \\
I_{2} & :=\sum_{k=1}^{d} \int_{(t-\epsilon) \vee s}^{t} d r \int_{0}^{1} d v \mathscr{S}_{2}^{2},
\end{aligned}
$$

and

$$
\begin{aligned}
& \mathscr{S}_{1}:=\sum_{i=1}^{d}\left(\lambda_{i}-\mu_{i}\right)\left[G_{s-r}(y, v) \sigma_{i, k}(u(r, v))+a_{i}(k, r, v, s, y)\right], \\
& \mathscr{S}_{2}:=\sum_{i=1}^{d} \mu_{i}\left[G_{t-r}(x, v) \sigma_{i k}(u(r, v))+a_{i}(k, r, v, t, x)\right] .
\end{aligned}
$$

From here on, Case 2 is divided into two further sub-cases.

Sub-Case A. Suppose, in addition, that $\epsilon \geq t-s$. In this case, we are going to prove that

$$
\inf _{\|\xi\|=1} \xi^{\top} \gamma_{Z} \xi \geq c \epsilon^{1 / 2}-Z_{1, \epsilon}
$$

where for all $q \geq 1$,

$$
\mathrm{E}\left[\left|Z_{1, \epsilon}\right|^{q}\right] \leq c(q) \epsilon^{q} .
$$

Indeed, we apply (4.4) to find that

$$
I_{1} \geq \frac{2}{3} \tilde{A}_{1}-B_{1}^{(1)}-B_{1}^{(2)},
$$

where

$$
\begin{aligned}
\tilde{A}_{1} & :=\sum_{k=1}^{d} \int_{s-\epsilon}^{s} d r \int_{0}^{1} d v\left(\sum_{i=1}^{d}\left[\left(\lambda_{i}-\mu_{i}\right) G_{s-r}(y, v)+\mu_{i} G_{t-r}(x, v)\right] \sigma_{i k}(u(r, v))\right)^{2}, \\
B_{1}^{(1)} & :=4\|\lambda-\mu\|^{2} \sum_{k=1}^{d} \int_{s-\epsilon}^{s} d r \int_{0}^{1} d v \sum_{i=1}^{d} a_{i}^{2}(k, r, v, s, y), \\
B_{1}^{(2)} & :=4\|\mu\|^{2} \sum_{k=1}^{d} \int_{s-\epsilon}^{s} d r \int_{0}^{1} d v \sum_{i=1}^{d} a_{i}^{2}(k, r, v, t, x) .
\end{aligned}
$$


Using the inequality

$$
(a+b)^{2} \geq a^{2}+b^{2}-2|a b|,
$$

we see that

$$
\tilde{A}_{1} \geq A_{1}+A_{2}-\left|B_{1}^{(3)}\right|,
$$

where

$$
\begin{aligned}
A_{1}:=\sum_{k=1}^{d} \int_{s-\epsilon}^{s} d r \int_{0}^{1} d v\left(\sum_{i=1}^{d}\left(\lambda_{i}-\mu_{i}\right) G_{s-r}(y, v) \sigma_{i k}(u(r, v))\right)^{2}, \\
A_{2}:=\sum_{k=1}^{d} \int_{s-\epsilon}^{s} d r \int_{0}^{1} d v\left(\sum_{i=1}^{d} \mu_{i} G_{t-r}(x, v) \sigma_{i k}(u(r, v))\right)^{2}, \\
B_{1}^{(3)}:=2 \sum_{k=1}^{d} \int_{s-\epsilon}^{s} d r \int_{0}^{1} d v\left(\sum_{i=1}^{d}\left(\lambda_{i}-\mu_{i}\right) G_{s-r}(y, v) \sigma_{i k}(u(r, v))\right) \\
\times\left(\sum_{i=1}^{d} \mu_{i} G_{t-r}(x, v) \sigma_{i k}(u(r, v))\right) .
\end{aligned}
$$

We can combine terms to find that

$$
I_{1} \geq \frac{2}{3}\left(A_{1}+A_{2}\right)-\left(B_{1}^{(1)}+B_{1}^{(2)}+\left|B_{1}^{(3)}\right|\right) .
$$

Finally, we appeal to (4.4) to find that

$$
I_{2} \geq \frac{2}{3} A_{3}-B_{2},
$$

where

$$
\begin{aligned}
& A_{3}:=\sum_{k=1}^{d} \int_{(t-\epsilon) \vee s}^{t} d r \int_{0}^{1} d v\left(\sum_{i=1}^{d} \mu_{i} G_{t-r}(x, v) \sigma_{i k}(u(r, v))\right)^{2}, \\
& B_{2}:=2 \sum_{k=1}^{d} \int_{(t-\epsilon) \vee s}^{t} d r \int_{0}^{1} d v\left(\sum_{i=1}^{d} \mu_{i} a_{i}(k, r, v, t, x)\right)^{2} .
\end{aligned}
$$

By hypothesis P2,

$$
\begin{aligned}
A_{1}+A_{2}+A_{3} \geq \rho^{2}( & \|\lambda-\mu\|^{2} \int_{s-\epsilon}^{s} d r \int_{0}^{1} d v G_{s-r}^{2}(y, v) \\
& +\|\mu\|^{2} \int_{s-\epsilon}^{s} d r \int_{0}^{1} d v G_{t-r}^{2}(x, v) \\
& \left.+\|\mu\|^{2} \int_{s}^{t} d r \int_{0}^{1} d v G_{t-r}^{2}(x, v)\right) .
\end{aligned}
$$


Note that we have used the defining assumption of Sub-Case A, namely, that $\epsilon \geq t-s$. Next, we group the last two integrals and apply Lemma 7.2 to find that

$$
\begin{aligned}
A_{1}+A_{2}+A_{3} & \geq c\left(\|\lambda-\mu\|^{2} \epsilon^{1 / 2}+\|\mu\|^{2} \int_{t-\epsilon}^{t} d r \int_{0}^{1} d v G_{t-r}^{2}(x, v)\right) \\
& \geq c\left(\|\lambda-\mu\|^{2}+\|\mu\|^{2}\right) \epsilon^{1 / 2} \\
& \geq c \epsilon^{1 / 2} .
\end{aligned}
$$

We are aiming for (6.15), and propose to bound the absolute moments of $B_{1}^{(i)}, i=1,2,3$ and $B_{2}$, separately. According to Lemma 6.11 below with $s=t$,

$$
\mathrm{E}\left[\sup _{\|\xi\|=1}\left|B_{2}\right|^{q}\right] \leq c(q) \epsilon^{q} .
$$

Next we bound the absolute moments of $B_{1}^{(i)}, i=1,2,3$. Using hypothesis $\mathbf{P} 1$ and Lemma 6.11, with $t=s$, we find that for all $q \geq 1$,

$$
\mathrm{E}\left[\sup _{\|\xi\|=1}\left|B_{1}^{(1)}\right|^{q}\right] \leq c \epsilon^{q} .
$$

In the same way, we see that

$$
\mathrm{E}\left[\sup _{\|\xi\|=1}\left|B_{1}^{(2)}\right|^{q}\right] \leq c(t-s+\epsilon)^{q / 2} \epsilon^{q / 2} .
$$

We are in the sub-case A where $t-s \leq \epsilon$. Therefrom, we obtain the following:

$$
\mathrm{E}\left[\sup _{\|\xi\|=1}\left|B_{1}^{(2)}\right|^{q}\right] \leq c \epsilon^{q} .
$$

We can combine (6.22) and (6.24) as follows:

$$
\mathrm{E}\left[\sup _{\|\xi\|=1}\left(B_{1}^{(1)}+B_{1}^{(2)}\right)^{q}\right] \leq c(q) \epsilon^{q} .
$$

Finally, we turn to bounding the absolute moments of $B_{1}^{(3)}$. Hypothesis $\mathbf{P} 1$ assures us that

$$
\begin{aligned}
\left|B_{1}^{(3)}\right| & \leq c \int_{s-\epsilon}^{s} d r \int_{0}^{1} d v G_{s-r}(y, v) G_{t-r}(x, v) \\
& =c \int_{s-\epsilon}^{s} d r G_{t+s-2 r}(x, y),
\end{aligned}
$$


thanks to the semi-group property WALSH [W86, (3.6)] (see (6.40) below). This and Lemma 7.1 together prove that

$$
\begin{aligned}
\left|B_{1}^{(3)}\right| & \leq c \int_{0}^{\epsilon} \frac{d u}{\sqrt{t-s+2 u}} \exp \left(-\frac{|x-y|^{2}}{2(t-s+2 u)}\right) \\
& \leq c \int_{0}^{\epsilon} \frac{d u}{\sqrt{t-s+2 u}} \exp \left(-\frac{\alpha^{2} \epsilon}{2(t-s+2 u)}\right),
\end{aligned}
$$

since $|x-y| \geq 2(1+\alpha) \epsilon^{1 / 2} \geq \alpha \epsilon^{1 / 2}$. Now we can change variables $\left[z:=2(t-s+2 u) /\left(\alpha^{2} \epsilon\right)\right]$, and use the bounds $0 \leq t-s \leq \epsilon$ to find that

$$
\left|B_{1}^{(3)}\right| \leq c \epsilon^{1 / 2} \Psi(\alpha), \quad \text { where } \quad \Psi(\alpha):=\alpha \int_{0}^{6 / \alpha^{2}} z^{-1 / 2} e^{-1 / z} d z .
$$

Now, by (6.20), (6.21), (6.26) and (6.25)

$$
\begin{aligned}
\inf _{\|\xi\|=1} \xi^{\top} \gamma_{Z} \xi & \geq \frac{2}{3}\left(A_{1}+A_{2}+A_{3}\right)-\left(B_{1}^{(1)}+B_{1}^{(2)}+\left|B_{1}^{(3)}\right|+B_{2}\right) \\
& \geq c_{1} \epsilon^{1 / 2}-c_{2} \Psi(\alpha) \epsilon^{1 / 2}-Z_{1, \epsilon}
\end{aligned}
$$

where $Z_{1, \epsilon}:=B_{1}^{(1)}+B_{1}^{(2)}+B_{2}$ satisfies $\mathrm{E}\left[\left|Z_{1, \epsilon}\right|^{q}\right] \leq c_{1}(q) \epsilon^{q}$. Because $\lim _{\nu \rightarrow \infty} \Psi(\nu)=0$, we can choose and fix $\alpha$ so large that $c_{2} \Psi(\alpha) \leq c_{1} / 4$ for the $c_{1}$ and $c_{2}$ of the preceding displayed equation. This yields,

$$
\inf _{\|\xi\|=1} \xi^{\top} \gamma_{Z} \xi \geq c \epsilon^{1 / 2}-Z_{1, \epsilon}
$$

as in (6.15) and (6.16).

Sub-Case B. In this final (sub-) case we suppose that $\epsilon \leq t-s \leq|x-y|^{2}$. Choose and fix $0<\epsilon<t-s$. During the course of our proof of Case 1, we established the following:

$$
\inf _{\|\xi\|=1} \xi^{\top} \gamma_{Z} \xi \geq \min \left(c \epsilon^{\frac{1}{2}+\eta}-Y_{1, \epsilon}, c \epsilon^{1 / 2}-Y_{2, \epsilon}\right)
$$

where

$$
\mathrm{E}\left[\left|Y_{1, \epsilon}\right|^{q}\right] \leq c(q) \epsilon^{q} \quad \text { and } \quad \mathrm{E}\left[\left|Y_{2, \epsilon}\right|^{q}\right] \leq c(q) \epsilon^{q\left(\frac{1}{2}+\eta\right)} .
$$

See (6.12) and (6.13).

Combine Sub-Cases A and B, and, in particular, (6.15) and (6.28), to find that for all $0<\epsilon<\frac{1}{4}(1+\alpha)^{-2}|x-y|^{2}$,

$$
\inf _{\|\xi\|=1} \xi^{\top} \gamma_{Z} \xi \geq \min \left(c \epsilon^{\frac{1}{2}+\eta}-Y_{1, \epsilon}, c \epsilon^{1 / 2}-Y_{2, \epsilon}-Z_{1, \epsilon} \mathbf{1}_{\{t-s<\epsilon\}}\right) .
$$

Because of this and (6.16), Proposition 3.5 implies that

$$
\begin{aligned}
\mathrm{E}\left[\left(\inf _{\|\xi\|=1} \xi^{\top} \gamma_{Z} \xi\right)^{-2 p d}\right] & \leq c|x-y|^{2(-2 d p)\left(\frac{1}{2}+\eta\right)} \\
& \leq c\left(|t-s|^{1 / 2}+|x-y|\right)^{-2 d p(1+2 \eta)}
\end{aligned}
$$


This concludes the proof of Proposition 6.9(a).

If $t=s$, then Sub-Case B does not arise, and so we get directly from (6.27) and Proposition 3.5 that

$$
\mathrm{E}\left[\left(\inf _{\|\xi\|=1} \xi^{\top} \gamma_{Z} \xi\right)^{-2 p d}\right] \leq c|x-y|^{-2 d p}
$$

This proves (b) and concludes the proof of Proposition 6.9.

Remark 6.10. If $\sigma$ and $b$ are constant, then $a_{i}=0$, so $\eta$ can be taken to be 0 . This gives the correct upper bound in the Gaussian case, which shows that the method of proof of Proposition 6.9 is rather tight.

We finally prove a result that we have used in the proof of Proposition 6.9.

Lemma 6.11. Assume $\mathbf{P} 1$. For all $T>0$ and $q \geq 1$, there exists a constant $c=c(q, T) \in$ $(0, \infty)$ such that for every $0<\epsilon \leq s \leq t \leq T$ and $x \in[0,1]$,

$$
\mathrm{E}\left[\left(\sum_{k=1}^{d} \int_{s-\epsilon}^{s} d r \int_{0}^{1} d v \sum_{i=1}^{d} a_{i}^{2}(k, r, v, t, x)\right)^{q}\right] \leq c(t-s+\epsilon)^{q / 2} \epsilon^{q / 2} .
$$

Proof. Define

$$
A:=\sum_{k=1}^{d} \int_{s-\epsilon}^{s} d r \int_{0}^{1} d v \sum_{i=1}^{d} a_{i}^{2}(k, r, v, t, x)
$$

Use (4.3) to write

$$
\mathrm{E}\left[|A|^{q}\right] \leq c\left(\mathrm{E}\left[\left|A_{1}\right|^{q}\right]+\mathrm{E}\left[\left|A_{2}\right|^{q}\right]\right),
$$

where

$$
A_{1}:=\sum_{i, j, k=1}^{d} \int_{s-\epsilon}^{s} d r \int_{0}^{1} d v\left|\int_{r}^{t} \int_{0}^{1} G_{t-\theta}(x, \eta) D_{r, v}^{(k)}\left(\sigma_{i j}(u(\theta, \eta))\right) W^{j}(d \theta, d \eta)\right|^{2},
$$

and

$$
A_{2}:=\sum_{i, k=1}^{d} \int_{s-\epsilon}^{s} d r \int_{0}^{1} d v\left|\int_{r}^{t} d \theta \int_{0}^{1} d \eta G_{t-\theta}(x, \eta) D_{r, v}^{(k)}\left(b_{i}(u(\theta, \eta))\right)\right|^{2} .
$$

We bound the $q$-th moment of $A_{1}$ and $A_{2}$ separately.

As regards $A_{1}$, we apply the Burkholder inequality for Hilbert-space-valued martingales (Lemma 7.6) to find that

$$
\mathrm{E}\left[\left|A_{1}\right|^{q}\right] \leq c \sum_{i, j, k=1}^{d} \mathrm{E}\left[\left|\int_{s-\epsilon}^{t} d \theta \int_{0}^{1} d \eta \int_{s-\epsilon}^{s} d r \int_{0}^{1} d v \Theta^{2}\right|^{q}\right]
$$

where

$$
\begin{aligned}
\Theta & :=\mathbf{1}_{\{\theta>r\}} G_{t-\theta}(x, \eta)\left|D_{r, v}^{(k)}\left(\sigma_{i j}(u(\theta, \eta))\right)\right| \\
& \leq c \mathbf{1}_{\{\theta>r\}} G_{t-\theta}(x, \eta)\left|\sum_{l=1}^{d} D_{r, v}^{(k)}\left(u_{l}(\theta, \eta)\right)\right|
\end{aligned}
$$


thanks to hypothesis P1. Thus,

$$
\mathrm{E}\left[\left|A_{1}\right|^{q}\right] \leq c \sum_{k=1}^{d} \mathrm{E}\left[\left|\int_{s-\epsilon}^{t} d \theta \int_{0}^{1} d \eta G_{t-\theta}^{2}(x, \eta) \int_{s-\epsilon}^{s \wedge \theta} d r \int_{0}^{1} d v\left(\sum_{l=1}^{d} D_{r, v}^{(k)}\left(u_{l}(\theta, \eta)\right)\right)^{2}\right|^{q}\right] .
$$

We apply Hölder's inequality with respect to the measure $G_{t-\theta}^{2}(x, \eta) d \theta d \eta$ to find that

$$
\begin{aligned}
\mathrm{E}\left[\left|A_{1}\right|^{q}\right] \leq c\left(\int_{s-\epsilon}^{t}\right. & \left.d \theta \int_{0}^{1} d \eta G_{t-\theta}^{2}(x, \eta)\right)^{q-1} \\
& \times \int_{s-\epsilon}^{t} d \theta \int_{0}^{1} d \eta G_{t-\theta}^{2}(x, \eta) \sum_{k=1}^{d} \mathrm{E}\left[\left|\int_{s-\epsilon}^{s \wedge \theta} d r \int_{0}^{1} d v \Upsilon^{2}\right|^{q}\right],
\end{aligned}
$$

where $\Upsilon:=\sum_{l=1}^{d} D_{r, v}^{(k)}\left(u_{l}(\theta, \eta)\right)$. Lemma 7.3 assures us that

$$
\left(\int_{s-\epsilon}^{t} d \theta \int_{0}^{1} d \eta G_{t-\theta}^{2}(x, \eta)\right)^{q-1} \leq c(t-s+\epsilon)^{(q-1) / 2}
$$

On the other hand, Lemma 7.5 implies that

$$
\sum_{k=1}^{d} \mathrm{E}\left[\left|\int_{s-\epsilon}^{s \wedge \theta} d r \int_{0}^{1} d v \Upsilon^{2}\right|^{q}\right] \leq c \epsilon^{q / 2}
$$

where $c \in(0, \infty)$ does not depend on $(\theta, \eta, s, t, \epsilon, x)$. Consequently,

$$
\begin{array}{rl}
\int_{s-\epsilon}^{t} & d \theta \int_{0}^{1} d \eta G_{t-\theta}^{2}(x, \eta) \sum_{k=1}^{d} \mathrm{E}\left[\left|\int_{s-\epsilon}^{s \wedge \theta} d r \int_{0}^{1} d v \Upsilon^{2}\right|^{q}\right] \\
& \leq c \epsilon^{q / 2} \int_{s-\epsilon}^{t} d \theta \int_{0}^{1} d \eta G_{t-\theta}^{2}(x, \eta) \\
& \leq c \epsilon^{q / 2}(t-s+\epsilon)^{1 / 2} .
\end{array}
$$

Equations (6.30), (6.31), and (6.32) together imply that

$$
\mathrm{E}\left[\left|A_{1}\right|^{q}\right] \leq c(t-s+\epsilon)^{q / 2} \epsilon^{q / 2} .
$$

This is the desired bound for the $q$-th moment of $A_{1}$. Next we derive a similar bound for $A_{2}$. This will finish the proof. By the Cauchy-Schwarz inequality

$$
\mathrm{E}\left[\left|A_{2}\right|^{q}\right] \leq c(t-s+\epsilon)^{q} \sum_{i, k=1}^{d} \mathrm{E}\left[\left|\int_{s-\epsilon}^{s} d r \int_{0}^{1} d v \int_{r}^{t} d \theta \int_{0}^{1} d \eta \Phi^{2}\right|^{q}\right]
$$

where $\Phi:=G_{t-\theta}(x, \eta)\left|D_{r, v}^{(k)}\left(b_{i}(u(\theta, \eta))\right)\right|$. From here on, the $q$-th moment of $A_{2}$ is estimated as that of $A_{1}$ was; cf. (6.29), and this yields $\mathrm{E}\left[\left|A_{2}\right|^{q}\right] \leq c(t-s+\epsilon)^{3 q / 2} \epsilon^{q / 2}$. This completes the proof.

Remark 6.12. It is possible to prove that $\mathrm{E}\left[\left|A_{1}\right|\right]$ is at least a constant times $(t-s+\epsilon)^{1 / 2} \epsilon^{1 / 2}$. In this sense, the preceding result is not improvable. 


\subsubsection{Large Eigenvalues}

Let $I$ and $J$ be two compact intervals as in Theorem 1.1.

Proposition 6.13. Assume $\mathbf{P} 1$ and $\mathbf{P 2}$. Fix $T>0$ and $p>1$. Then there exists $C=$ $C(p, T)$ such that for all $s, t \in I$ with $0 \leq t-s<\frac{1}{2}, x, y \in J,(s, y) \neq(t, x)$,

$$
\mathrm{E}\left[\mathbf{1}_{A_{K}}\left(\prod_{i \in K}\left(\xi^{i}\right)^{T} \gamma_{Z} \xi^{i}\right)^{-p}\right] \leq C,
$$

where $A_{K}$ is defined as in (6.11).

Proof. Let $0<\epsilon<s \leq t$. We fix $i_{0} \in\{1, \ldots, 2 d\}$ and write $\tilde{\lambda}^{i_{0}}=\left(\tilde{\lambda}_{1}^{i_{0}}, \ldots, \tilde{\lambda}_{d}^{i_{0}}\right)$ and $\tilde{\mu}^{i_{0}}=\left(\tilde{\mu}_{1}^{i_{0}}, \ldots, \tilde{\mu}_{d}^{i_{0}}\right)$. We look at $\left(\xi^{i_{0}}\right)^{\top} \gamma_{Z} \xi^{i_{0}}$ on the event $\left\{\alpha_{i_{0}} \geq \alpha_{0}\right\}$. As in the proof of Proposition 6.9 and using the notation from (6.10), this is bounded below by

$$
\begin{aligned}
& \sum_{k=1}^{d} \int_{s-\epsilon}^{s} d r \int_{0}^{1} d v\left(\sum _ { i = 1 } ^ { d } \left[\left(\alpha_{i_{0}} \tilde{\lambda}_{i}^{i_{0}} G_{s-r}(y, v)\right.\right.\right. \\
& \left.\quad+\tilde{\mu}_{i}^{i_{0}} \sqrt{1-\alpha_{i_{0}}^{2}}\left(G_{t-r}(x, v)-G_{s-r}(y, v)\right)\right) \sigma_{i k}(u(r, v)) \\
& \quad+\alpha_{i_{0}} \tilde{\lambda}_{i}^{i_{0}} a_{i}(k, r, v, s, y) \\
& \left.\left.\quad+\tilde{\mu}_{i}^{i_{0}} \sqrt{1-\alpha_{i_{0}}^{2}}\left(a_{i}(k, r, v, t, x)-a_{i}(k, r, v, s, y)\right)\right]\right)^{2} \\
& +\sum_{k=1}^{d} \int_{s \vee(t-\epsilon)}^{t} d r \int_{0}^{1} d v\left(\sum _ { i = 1 } ^ { d } \left[\tilde{\mu}_{i}^{i_{0}} \sqrt{1-\alpha_{i_{0}}^{2}} G_{t-r}(x, v) \sigma_{i k}(u(r, v))\right.\right. \\
& \left.\left.\quad+\tilde{\mu}_{i}^{i_{0}} \sqrt{1-\alpha_{i_{0}}^{2}} a_{i}(k, r, v, t, x)\right]\right)^{2} .
\end{aligned}
$$

We intend to use Proposition 3.5 with $\varepsilon_{0}>0$ fixed, so we seek lower bounds for this expression for $0<\varepsilon<\varepsilon_{0}$. In the remainder of this proof, we will use the generic notation $\alpha, \tilde{\lambda}$ and $\tilde{\mu}$ for the realisations $\alpha_{i_{0}}(\omega), \tilde{\lambda}^{i_{0}}(\omega)$, and $\tilde{\mu}^{i_{0}}(\omega)$.

Case 1. $t-s \leq \epsilon$. Then, by (4.4), the expression in (6.35) is bounded below by

$$
\frac{2}{3}\left(f_{1}(s, t, \epsilon, \alpha, \tilde{\lambda}, \tilde{\mu}, x, y)+f_{2}(s, t, \epsilon, \alpha, \tilde{\lambda}, \tilde{\mu}, x, y)\right)-2 I_{\epsilon},
$$

where, from hypothesis P2,

$$
\begin{aligned}
& f_{1} \geq c \rho^{2} \int_{s-\epsilon}^{s} d r \int_{0}^{1} d v\left\|\alpha \tilde{\lambda} G_{s-r}(y, v)+\sqrt{1-\alpha^{2}} \tilde{\mu}\left(G_{t-r}(x, v)-G_{s-r}(y, v)\right)\right\|^{2}, \\
& f_{2} \geq c \rho^{2} \int_{s \vee(t-\epsilon)}^{t} d r \int_{0}^{1} d v\left\|\tilde{\mu} \sqrt{1-\alpha^{2}} G_{t-r}(x, v)\right\|^{2},
\end{aligned}
$$


and $I_{\epsilon}=3\left(I_{1, \epsilon}+I_{2, \epsilon}+I_{3, \epsilon}\right)$, where

$$
\begin{aligned}
I_{1, \epsilon} & :=\sum_{k=1}^{d} \int_{s-\epsilon}^{s} d r \int_{0}^{1} d v\left(\sum_{i=1}^{d}\left[\alpha \tilde{\lambda}_{i}-\tilde{\mu}_{i} \sqrt{1-\alpha^{2}}\right] a_{i}(k, r, v, s, y)\right)^{2}, \\
I_{2, \epsilon} & :=\sum_{k=1}^{d} \int_{s-\epsilon}^{s} d r \int_{0}^{1} d v\left(\sum_{i=1}^{d} \tilde{\mu}_{i} \sqrt{1-\alpha^{2}} a_{i}(k, r, v, t, x)\right)^{2}, \\
I_{3, \epsilon} & :=\sum_{k=1}^{d} \int_{t-\epsilon}^{t} d r \int_{0}^{1} d v\left(\sum_{i=1}^{d} \tilde{\mu}_{i} \sqrt{1-\alpha^{2}} a_{i}(k, r, v, t, x)\right)^{2} .
\end{aligned}
$$

There are obvious similarities between the terms $I_{1, \epsilon}$ and $B_{1}^{(1)}$ in (6.17). However, we must keep in mind that $\alpha, \tilde{\lambda}$ and $\tilde{\mu}$ are realisations of $\alpha_{i_{0}}, \tilde{\lambda}^{i_{0}}$ and $\tilde{\mu}^{i_{0}}$. Therefore,

$$
\begin{aligned}
I_{1, \epsilon} & =\sum_{k=1}^{d} \int_{s-\epsilon}^{s} d r \int_{0}^{1} d v\left(\sum_{i=1}^{d}\left[\alpha_{i_{0}} \tilde{\lambda}_{i}^{i_{0}}-\tilde{\mu}_{i}^{i_{0}} \sqrt{1-\alpha^{2}}\right] a_{i}(k, r, v, s, y)\right)^{2}, \\
& \leq C \sum_{k=1}^{d} \int_{s-\epsilon}^{s} d r \int_{0}^{1} d v \sum_{i=1}^{d} a_{i}^{2}(k, r, v, s, y) .
\end{aligned}
$$

Thus, we apply the same method that was used to bound $\mathrm{E}\left[\left|B_{1}^{(1)}\right|^{q}\right]$ to deduce that $\mathrm{E}\left[\left|I_{1, \epsilon}\right|^{q}\right] \leq$ $c(q) \epsilon^{q}$. Similarly, since $I_{2, \epsilon}$ is similar to $B_{1}^{(2)}$ from (6.18) and $t-s \leq \epsilon$, we see using (6.24) that $\mathrm{E}\left[\left|I_{2, \epsilon}\right|^{q}\right] \leq c(q) \epsilon^{q}$. Finally, using the similarity between $I_{3, \epsilon}$ and $B_{2}$ in (6.19), we see that $\mathrm{E}\left[\left|I_{3, \epsilon}\right|^{q}\right] \leq c(q) \epsilon^{q}$.

We claim that for every $\alpha_{0}>0$, there exists $\epsilon_{0}>0$ and $c_{0}>0$ such that

$$
f_{1}+f_{2} \geq c_{0} \sqrt{\epsilon} \text { for all } \alpha \in\left[\alpha_{0}, 1\right], \epsilon \in\left(0, \epsilon_{0}\right], s, t \in[1,2], x, y \in[0,1] .
$$

Using this for the $\alpha_{0}$ from Lemma 6.8, this will imply in particular that for $\epsilon \geq t-s$,

$$
\left(\xi^{i_{0}}\right)^{\top} \gamma_{Z} \xi^{i_{0}} \geq c_{0} \epsilon^{1 / 2}-2 I_{\epsilon}
$$

where $\mathrm{E}\left[\left|I_{\epsilon}\right|^{q}\right] \leq c(q) \epsilon^{q}$.

In order to prove (6.38), first define

$$
p_{t}(x, y):=(4 \pi t)^{-1 / 2} e^{-(x-y)^{2} /(4 t)} .
$$

In addition, let $g_{1}(s, t, \epsilon, \alpha, \tilde{\lambda}, \tilde{\mu}, x, y)$ and $g_{2}(s, t, \epsilon, \alpha, \tilde{\lambda}, \tilde{\mu}, x, y)$ be defined by the same expressions as the right-hand sides of (6.36) and (6.37), but with $G_{s-r}(x, v)$ replaced by $p_{s-r}(x-v)$, and $\int_{0}^{1}$ replaced by $\int_{-\infty}^{+\infty}$.

Observe that $g_{1} \geq 0, g_{2} \geq 0$, and if $g_{1}=0$, then for all $v \in \mathbb{R}$,

$$
\left\|\alpha p_{s-r}(y-v) \tilde{\lambda}+\sqrt{1-\alpha^{2}}\left(p_{t-r}(x-v)-p_{s-r}(y-v)\right) \tilde{\mu}\right\|=0 .
$$

If, in addition, $\tilde{\lambda}=\tilde{\mu}$, then we get that for all $v \in \mathbb{R}$,

$$
\left(\alpha-\sqrt{1-\alpha^{2}}\right) p_{s-r}(y-v)+\sqrt{1-\alpha^{2}} p_{t-r}(x-v)=0 .
$$


We take Fourier transforms to deduce from this that for all $\xi \in \mathbb{R}$,

$$
\left(\alpha-\sqrt{1-\alpha^{2}}\right) e^{i \xi y}=-\sqrt{1-\alpha^{2}} e^{i \xi x} e^{(s-t) \xi^{2}} .
$$

If $x=y$, then it follows that $s=t$ and $\alpha-\sqrt{1-\alpha^{2}}=-\sqrt{1-\alpha^{2}}$. Hence, if $\alpha \neq 0, x=y$ and $\tilde{\lambda}=\tilde{\mu}$, then $g_{1}>0$. We shall make use of this observation shortly.

Because $\|\tilde{\lambda}\|=\|\tilde{\mu}\|=1, f_{1}$ is bounded below by

$$
\begin{aligned}
& c \rho^{2} \int_{s-\epsilon}^{s} d r \int_{0}^{1} d v\left(\alpha^{2} G_{s-r}^{2}(y, v)+\left(1-\alpha^{2}\right)\left(G_{t-r}(x, v)-G_{s-r}(y, v)\right)^{2}\right. \\
& \left.\quad+2 \alpha \sqrt{1-\alpha^{2}} G_{s-r}(y, v)\left(G_{t-r}(x, v)-G_{s-r}(y, v)\right)(\tilde{\lambda} \cdot \tilde{\mu})\right) \\
& =c \rho^{2} \int_{s-\epsilon}^{s} d r \int_{0}^{1} d v\left(\left(\alpha-\sqrt{1-\alpha^{2}}\right)^{2} G_{s-r}^{2}(y, v)\right)+\left(1-\alpha^{2}\right) G_{t-r}^{2}(x, v) \\
& \quad+2\left(\alpha-\sqrt{1-\alpha^{2}}\right) \sqrt{1-\alpha^{2}} G_{s-r}(y, v) G_{t-r}(x, v) \\
& \left.\quad+2 \alpha \sqrt{1-\alpha^{2}} G_{s-r}(y, v)\left(G_{t-r}(x, v)-G_{s-r}(y, v)\right)(\tilde{\lambda} \cdot \tilde{\mu}-1)\right) .
\end{aligned}
$$

Recall the semigroup property

$$
\int_{0}^{1} d v G_{s-r}(y, v) G_{t-r}(x, v)=G_{s+t-2 r}(x, y)
$$

(see WALSh [W86, (3.6)]). We set $h:=t-s$ and change variables $[\bar{r}:=s-r]$ to obtain the following bound:

$$
\begin{aligned}
f_{1} \geq c \rho^{2} \int_{0}^{\epsilon} d r\left(\left(\alpha-\sqrt{1-\alpha^{2}}\right)^{2} G_{2 r}(y, y)+\left(1-\alpha^{2}\right) G_{2 h+2 r}(x, x)\right. \\
\quad+2\left(\alpha-\sqrt{1-\alpha^{2}}\right) \sqrt{1-\alpha^{2}} G_{h+2 r}(x, y) \\
\left.\quad+2 \alpha \sqrt{1-\alpha^{2}}\left(G_{h+2 r}(x, y)-G_{2 r}(y, y)\right)(\tilde{\lambda} \cdot \tilde{\mu}-1)\right) .
\end{aligned}
$$

Recall ([W86, p.318]), that

$$
G_{t}(x, y)=p_{t}(x, y)+H_{t}(x, y),
$$

where $H_{t}(x, y)$ is a continuous function that is uniformly bounded over $(t, x, y) \in(0, \infty) \times$ $(0,1) \times(0,1)$. Therefore, $f_{1} \geq c \rho^{2} \tilde{g}_{1}-c \epsilon$, where

$$
\begin{aligned}
\tilde{g}_{1}:=\tilde{g}_{1}(h, \epsilon, \alpha, \tilde{\lambda}, \tilde{\mu}, x, y) \\
=\int_{0}^{\epsilon} d r\left(\left(\alpha-\sqrt{1-\alpha^{2}}\right)^{2} p_{2 r}(y, y)+\left(1-\alpha^{2}\right) p_{2 h+2 r}(x, x)\right. \\
+2\left(\alpha-\sqrt{1-\alpha^{2}}\right) \sqrt{1-\alpha^{2}} p_{h+2 r}(x, y) \\
\left.+2 \alpha \sqrt{1-\alpha^{2}}\left(p_{h+2 r}(x, y)-p_{2 r}(y, y)\right)(\tilde{\lambda} \cdot \tilde{\mu}-1)\right) .
\end{aligned}
$$


We can recognize that

$$
p_{h+2 r}(x, y)-p_{2 r}(y, y)=\frac{\exp \left(-(x-y)^{2} /(4(h+2 r))\right)}{\sqrt{4 \pi(h+2 r)}}-\frac{1}{\sqrt{4 \pi(2 r)}} \leq 0 .
$$

Also, $\tilde{\lambda} \cdot \tilde{\mu}-1 \leq 0$. Thus,

$$
\tilde{g_{1}} \geq \hat{g_{1}}
$$

where

$$
\begin{aligned}
\hat{g_{1}}:=\hat{g_{1}}(h, \epsilon, \alpha, x, y) & \\
=\int_{0}^{\epsilon} d r & \left(\left(\alpha-\sqrt{1-\alpha^{2}}\right)^{2} p_{2 r}(y, y)+\left(1-\alpha^{2}\right) p_{2 h+2 r}(x, x)\right. \\
& \left.+2\left(\alpha-\sqrt{1-\alpha^{2}}\right) \sqrt{1-\alpha^{2}} p_{h+2 r}(x, y)\right) .
\end{aligned}
$$

Therefore,

$$
\begin{gathered}
\hat{g_{1}}=\int_{0}^{\epsilon} d r\left(\left(\alpha-\sqrt{1-\alpha^{2}}\right)^{2} \frac{1}{\sqrt{8 \pi r}}+\left(1-\alpha^{2}\right) \frac{1}{\sqrt{8 \pi(h+r)}}\right. \\
\left.+2\left(\alpha-\sqrt{1-\alpha^{2}}\right) \sqrt{1-\alpha^{2}} p_{h+2 r}(x, y)\right) .
\end{gathered}
$$

On the other hand, by (6.40) above,

$$
\begin{aligned}
f_{2} & \geq \int_{0}^{\epsilon \wedge(t-s)} d r\left(1-\alpha^{2}\right) G_{2 r}(y, y) \\
& \geq \tilde{g}_{2}:=\int_{0}^{\epsilon \wedge h} d r\left(1-\alpha^{2}\right) p_{2 r}(y, y)-C \epsilon \\
& =\left(1-\alpha^{2}\right) \sqrt{\epsilon \wedge h}-C \epsilon .
\end{aligned}
$$

Finally, we conclude that

$$
\begin{aligned}
f_{1}+f_{2} \geq \hat{g}_{1} & +\tilde{g}_{2}-2 C \epsilon \\
=( & \left.\alpha-\sqrt{1-\alpha^{2}}\right)^{2} \frac{\sqrt{\epsilon}}{\sqrt{2 \pi}}+\frac{1-\alpha^{2}}{\sqrt{2 \pi}}(\sqrt{h+\epsilon}-\sqrt{h}) \\
& +2\left(\alpha-\sqrt{1-\alpha^{2}}\right) \sqrt{1-\alpha^{2}} \int_{0}^{\epsilon} d r p_{h+2 r}(x, y) \\
& +\frac{1-\alpha^{2}}{\sqrt{2 \pi}} \sqrt{\epsilon \wedge h}-2 C \epsilon .
\end{aligned}
$$

Now we consider two different sub-cases.

Sub-case (i). Suppose $\alpha-\sqrt{1-\alpha^{2}} \geq 0$, that is, $\alpha \geq 2^{-1 / 2}$. Then

$$
\epsilon^{-1 / 2}\left(\hat{g_{1}}+\tilde{g}_{2}\right) \geq \phi_{1}\left(\alpha, \frac{h}{\epsilon}\right)-2 C \epsilon^{1 / 2}
$$


where

$$
\phi_{1}(\alpha, z):=\frac{1}{\sqrt{2 \pi}}\left(\left(\alpha-\sqrt{1-\alpha^{2}}\right)^{2}+\left(1-\alpha^{2}\right) \frac{1}{\sqrt{1+z}+\sqrt{z}}+\left(1-\alpha^{2}\right) \sqrt{1 \wedge z}\right) .
$$

Clearly,

$$
\begin{aligned}
\inf _{\alpha \geq 2^{-1 / 2}} \inf _{z>0} \phi_{1}(\alpha, z) & \geq \inf _{\alpha>2^{-1 / 2}} \frac{\left(\alpha-\sqrt{1-\alpha^{2}}\right)^{2}+c_{0}\left(1-\alpha^{2}\right)}{\sqrt{2 \pi}} \\
& >\phi_{0}>0 .
\end{aligned}
$$

Thus,

$$
\inf _{\alpha \geq 2^{-1 / 2}, h \geq 0,0<\epsilon \leq \epsilon_{0}} \epsilon^{-1 / 2}\left(\hat{g_{1}}+\tilde{g}_{2}\right)>0 .
$$

Sub-case (ii). Now we consider the case where $\alpha-\sqrt{1-\alpha^{2}}<0$, that is, $\alpha<2^{-1 / 2}$. In this case,

$$
\epsilon^{-1 / 2}\left(\hat{g_{1}}+\tilde{g}_{2}\right) \geq \psi_{1}\left(\alpha, \frac{h}{\epsilon}\right)-2 C \epsilon^{1 / 2}
$$

where

$$
\begin{aligned}
\psi_{1}(\alpha, z):= & \frac{1}{\sqrt{2 \pi}}\left(\left(\alpha-\sqrt{1-\alpha^{2}}\right)^{2}+\left(1-\alpha^{2}\right) \frac{1}{\sqrt{1+z}+\sqrt{z}}\right. \\
& \left.-2\left(\sqrt{1-\alpha^{2}}-\alpha\right) \sqrt{1-\alpha^{2}} \frac{\sqrt{2}}{\sqrt{2+z}+\sqrt{z}}+\left(1-\alpha^{2}\right) \sqrt{1 \wedge z}\right) .
\end{aligned}
$$

Note that $\psi_{1}(\alpha, z)>0$ if $\alpha \neq 0$. This corresponds to the observation made in the lines following (6.39). Moreover, for $z \geq 1$, we have $\psi_{1}(\alpha, z) \geq(2 \pi)^{-1 / 2} \alpha^{2}$ so that

$$
\inf _{\alpha \in\left[\alpha_{0}, 2^{-1 / 2}\right]} \inf _{z \geq 0} \psi_{1}(\alpha, z) \geq \min \left\{(2 \pi)^{-1 / 2} \alpha_{0}^{2}, \inf _{\alpha \in\left[\alpha_{0}, 2^{-1 / 2}\right]} \inf _{z \in[0,1]} \psi_{1}(\alpha, z)\right\} .
$$

Since $\lim _{z \downarrow 0} \psi_{1}(\alpha, z) \geq(2 \pi)^{-1 / 2} \alpha^{2} \geq(2 \pi)^{-1 / 2} \alpha_{0}^{2}$ and $\psi_{1}(\alpha, z)>0$, there exists $c_{\alpha_{0}}>0$ such that

$$
\inf _{\alpha \in\left[\alpha_{0}, 2^{-1 / 2}\right]} \inf _{z \in[0,1]} \psi_{1}(\alpha, z) \geq c_{\alpha_{0}},
$$

and hence

$$
\inf _{\alpha \in\left[\alpha_{0}, 2^{-1 / 2}\right], z \geq 0} \psi_{1}(\alpha, z)>0 .
$$

This concludes the proof of the claim (6.38).

Case 2. $t-s>\epsilon$. In accord with (6.35), we are interested in

$$
\inf _{1 \geq \alpha \geq \alpha_{0}}\left(\xi^{i_{0}}\right)^{\top} \gamma_{Z} \xi^{i_{0}}:=\min \left(E_{1, \epsilon}, E_{2, \epsilon}\right),
$$


where

$$
\begin{aligned}
& E_{1, \epsilon}:=\inf _{\alpha_{0} \leq \alpha \leq \sqrt{1-\epsilon^{\eta}}}\left(\xi^{i_{0}}\right)^{\top} \gamma_{Z} \xi^{i_{0}}, \\
& E_{2, \epsilon}:=\inf _{\sqrt{1-\epsilon^{\eta}} \leq \alpha \leq 1}\left(\xi^{i_{0}}\right)^{\top} \gamma_{Z} \xi^{i_{0}} .
\end{aligned}
$$

Clearly,

$$
E_{1, \epsilon} \geq \frac{2}{3} f_{2}-2 I_{3, \epsilon}
$$

Since $\alpha \leq \sqrt{1-\epsilon^{\eta}}$ is equivalent to $\sqrt{1-\alpha^{2}} \geq \epsilon^{\eta / 2}$, we use hypothesis $\mathbf{P 2}$ to deduce that

$$
f_{2} \geq c \rho^{2} \epsilon^{\eta} \int_{t-\epsilon}^{t} d r \int_{0}^{1} d v G_{t-r}^{2}(x, v) \geq c \rho^{2} \epsilon^{\frac{1}{2}+\eta} .
$$

Therefore,

$$
E_{1, \epsilon} \geq c \rho^{2} \epsilon^{\frac{1}{2}+\eta}-I_{3, \epsilon},
$$

and we have seen that $I_{3, \epsilon}$ has the desirable property $\mathrm{E}\left[\left|I_{3, \epsilon}\right|^{q}\right] \leq c(q) \epsilon^{q}$.

In order to estimate $E_{2, \epsilon}$, we observe using (6.35) that

$$
E_{2, \epsilon} \geq \frac{2}{3} \tilde{f}_{1}-\tilde{J}_{1, \epsilon}-\tilde{J}_{2, \epsilon}-\tilde{J}_{3, \epsilon}-\tilde{J}_{4, \epsilon}
$$

where

$$
\begin{aligned}
& \tilde{f}_{1} \geq \alpha^{2} \sum_{k=1}^{d} \int_{s-\epsilon}^{s} d r \int_{0}^{1} d v\left(\sum_{i=1}^{d} \tilde{\lambda}_{i} \sigma_{i k}(u(r, v))\right)^{2} G_{s-r}^{2}(y, v), \\
& \tilde{J}_{1, \epsilon}=2\left(1-\alpha^{2}\right) \sum_{k=1}^{d} \int_{s-\epsilon}^{s} d r \int_{0}^{1} d v\left(\sum_{i=1}^{d} \tilde{\mu}_{i} \sigma_{i k}(u(r, v))\right)^{2} G_{t-r}^{2}(x, v), \\
& \tilde{J}_{2, \epsilon}=2\left(1-\alpha^{2}\right) \sum_{k=1}^{d} \int_{s-\epsilon}^{s} d r \int_{0}^{1} d v\left(\sum_{i=1}^{d} \tilde{\mu}_{i} \sigma_{i k}(u(r, v))\right)^{2} G_{s-r}^{2}(y, v), \\
& \tilde{J}_{3, \epsilon}=2 \sum_{k=1}^{d} \int_{s-\epsilon}^{s} d r \int_{0}^{1} d v\left(\sum_{i=1}^{d}\left(\alpha \tilde{\lambda}_{i}-\tilde{\mu}_{i} \sqrt{1-\alpha^{2}}\right) a_{i}(k, r, v, s, y)\right)^{2}, \\
& \tilde{J}_{4, \epsilon}=2\left(1-\alpha^{2}\right) \sum_{k=1}^{d} \int_{s-\epsilon}^{s} d r \int_{0}^{1} d v\left(\sum_{i=1}^{d} \tilde{\mu}_{i} a_{i}(k, r, v, t, x)\right)^{2} .
\end{aligned}
$$

Because $\alpha^{2} \geq 1-\epsilon^{\eta}$ and $\epsilon \leq t-s \leq \frac{1}{2}$, hypothesis $\mathbf{P 2}$ and Lemma 7.2 imply that $\tilde{f}_{1} \geq c \epsilon^{1 / 2}$. On the other hand, since $1-\alpha^{2} \leq \epsilon^{\eta}$, we can use hypothesis $\mathbf{P} \mathbf{1}$ and Lemma 7.4 to see that

$$
\mathrm{E}\left[\left|\tilde{J}_{1, \epsilon}\right|^{q}\right] \leq c(q) \epsilon^{q \eta} \epsilon^{q / 2}=c(q) \epsilon^{\left(\frac{1}{2}+\eta\right) q},
$$

and similarly, using Lemma $7.3, \mathrm{E}\left[\left|\tilde{J}_{2, \epsilon}\right|^{q}\right] \leq c(q) \epsilon^{\left(\frac{1}{2}+\eta\right) q}$. The term $\tilde{J}_{3, \epsilon}$ is equal to $2 I_{1, \varepsilon}$, so $\mathrm{E}\left[\left|\tilde{J}_{3, \epsilon}\right|^{q}\right] \leq c \varepsilon^{q}$, and $\tilde{J}_{4, \varepsilon}$ is similar to $B_{1}^{(2)}$ from (6.18), so we find using (6.23) that

$$
\mathrm{E}\left[\left|\tilde{J}_{4, \epsilon}\right|^{q}\right] \leq c \epsilon^{q \eta}(t-s+\epsilon)^{q / 2} \epsilon^{q / 2} \leq c \epsilon^{\left(\frac{1}{2}+\eta\right) q} .
$$


We conclude that when $t-s>\epsilon$, then $E_{2, \epsilon} \geq c \epsilon^{1 / 2}-\tilde{J}_{\epsilon}$, where $\mathrm{E}\left[\left|\tilde{J}_{\epsilon}\right|^{q}\right] \leq c(q) \epsilon^{\left(\frac{1}{2}+\eta\right) q}$. Therefore, when $t-s>\epsilon$,

$$
1_{\left\{\alpha_{i_{0}} \geq \alpha_{0}\right\}}\left(\xi^{i_{0}}\right)^{\top} \gamma_{Z} \xi^{i_{0}} \geq 1_{\left\{\alpha_{i_{0}} \geq \alpha_{0}\right\}} \min \left(c \rho^{2} \epsilon^{\frac{1}{2}+\eta}-I_{3, \epsilon}, c \epsilon^{\frac{1}{2}}-\tilde{J}_{\epsilon}\right) .
$$

Putting together the results of Case 1 and Case 2, we see that for $0<\epsilon \leq \epsilon_{0}$,

$$
1_{\left\{\alpha_{i_{0}} \geq \alpha_{0}\right\}}\left(\xi^{i_{0}}\right)^{\top} \gamma_{Z} \xi^{i_{0}} \geq 1_{\left\{\alpha_{i_{0}} \geq \alpha_{0}\right\}} Z,
$$

where

$$
Z=\min \left(c \rho^{2} \epsilon^{\frac{1}{2}+\eta}-I_{3, \epsilon}, c \epsilon^{\frac{1}{2}}-2 I_{\epsilon} \mathbf{1}_{\{\epsilon \geq t-s\}}-\tilde{J}_{\epsilon} \mathbf{1}_{\{\epsilon<t-s\}}\right) .
$$

Note that all the constants are independent of $i_{0}$. Taking into account the bounds on moments of $I_{3, \epsilon}, I_{\epsilon}$ and $\tilde{J}_{\epsilon}$, and then using Proposition 3.5, we deduce that for all $p \geq 1$, there is $C>0$ such that

$$
E\left[\left(1_{\left\{\alpha_{i_{0}} \geq \alpha_{0}\right\}}\left(\xi^{i_{0}}\right)^{\top} \gamma_{Z} \xi^{i_{0}}\right)^{-p}\right] \leq E\left[1_{\left\{\alpha_{i_{0}} \geq \alpha_{0}\right\}} Z^{-p}\right] \leq E\left[Z^{-p}\right] \leq C .
$$

Since this applies to any $p \geq 1$, we can use Hölder's inequality to deduce (6.34). This proves Proposition 6.13.

\section{Appendix}

On several occasions, we have appealed to the following technical estimates on the Green kernel of the heat equation.

Lemma 7.1. [BP98, (A.1)] There exists $C>0$ such that for any $0<s<t$ and $x, y \in[0,1]$, $x \neq y$,

$$
G_{t-s}(x, y) \leq C \frac{1}{\sqrt{2 \pi(t-s)}} \exp \left(-\frac{|x-y|^{2}}{2(t-s)}\right) .
$$

Lemma 7.2. [BP98, (A.3)] There exists $C>0$ such that for any $t \geq \epsilon>0$ and $x \in[0,1]$,

$$
\int_{t-\epsilon}^{t} \int_{x-\sqrt{\epsilon}}^{x+\sqrt{\epsilon}} G_{t-s}^{2}(x, y) d y d s \geq C \sqrt{\epsilon}
$$

Lemma 7.3. [BP98, (A.5)] There exists $C>0$ such that for any $\epsilon>0, q<\frac{3}{2}, t \geq \epsilon$ and $x \in[0,1]$,

$$
\int_{t-\epsilon}^{t} \int_{0}^{1} G_{t-s}^{2 q}(x, y) d y d s \leq C \epsilon^{3 / 2-q} .
$$

Lemma 7.4. There exists $C>0$ such that for all $0<a<b$ and $x \in[0,1]$,

$$
\int_{a}^{b} \int_{0}^{1} G_{s}^{2}(x, y) d y d s \leq C \frac{b-a}{\sqrt{b}+\sqrt{a}}
$$


Proof. Using Lemma 7.1 and the change of variables $z=\frac{x-y}{\sqrt{s}}$, we see that

$$
\begin{aligned}
\int_{a}^{b} \int_{0}^{1} G_{s}^{2}(x, y) d y d s & \leq C \int_{a}^{b} \int_{-\infty}^{\infty} \frac{1}{\sqrt{s}} e^{-z^{2}} d z d s \\
& =\tilde{C} \int_{a}^{b} \frac{1}{\sqrt{s}} d s=2 \tilde{C}(\sqrt{b}-\sqrt{a})
\end{aligned}
$$

which concludes the proof.

The next result is a straightforward extension to $d \geq 1$ of MorIEn [M98, Lemma 4.2] for $d=1$.

Lemma 7.5. Assume P1. For all $q \geq 1, T>0$ there exists $C>0$ such that for all $T \geq t \geq s \geq \epsilon>0$ and $0 \leq y \leq 1$,

$$
\sum_{k=1}^{d} \mathrm{E}\left[\left(\int_{s-\epsilon}^{s} d r \int_{0}^{1} d v\left|\sum_{i=1}^{d} D_{r, v}^{(k)}\left(u_{i}(t, y)\right)\right|^{2}\right)^{q}\right] \leq C \epsilon^{q / 2}
$$

The next result is Burkholder's inequality for Hilbert-space-valued martingales.

Lemma 7.6. [BP98, eq.(4.18)] Let $H_{s, t}$ be a predictable $L^{2}\left(([0, t] \times[0,1])^{m}, d \alpha\right)$-valued process, where $m \geq 1$ and $d \alpha$ denotes Lebesgue measure. Then, for any $p \geq 1$, there exists $C>0$ such that

$$
\begin{aligned}
\mathrm{E}\left[\left|\int_{([0, t] \times[0,1])^{m}}\left(\int_{0}^{t} \int_{0}^{1} H_{s, y}(\alpha) W(d y, d s)\right)^{2} d \alpha\right|^{p}\right] \\
\leq C \mathrm{E}\left[\left|\int_{0}^{t} \int_{0}^{1}\left(\int_{([0, t] \times[0,1])^{m}} H_{s, y}^{2}(\alpha) d \alpha\right) d y d s\right|^{p}\right] .
\end{aligned}
$$

Acknowledgement. The authors thank V. Bally for several stimulating discussions and an anonymous referee for a careful reading of the manuscript and many useful comments.

\section{References}

[BMS95] Bally, V., Millet, A. And Sanz-Solé, M. (1995), Approximation and support theorem in Hölder norm for parabolic stochastic partial differential equations, The Annals of Probability, 23, 178-222.

[BP98] Bally, V. And Pardoux, E. (1998), Malliavin calculus for white noise driven parabolic SPDEs, Potential Analysis, 9, 27-64.

[DN04] Dalang, R.C. And Nualart, E. (2004), Potential theory for hyperbolic SPDEs, The Annals of Probability, 32, 2099-2148. 
[DKN07] Dalang, R.C., Khoshnevisan, D. and Nualart, E. (2007), Hitting probabilities for systems of non-linear stochastic heat equations with additive noise, $A L E A, \mathbf{3}, 231-271$.

[K85] Kahane, J.-P. (1985), Some random series of functions, Cambridge University Press.

[K02] Khoshnevisan, D. (2002), Multiparameter processes. An introduction to random fields, Springer-Verlag.

[K03] Kohatsu-Higa, A. (2003), Lower bound estimates for densities of uniformly elliptic random variables on Wiener space, Probab. Theory Related Fields, 126, 421-457.

[M98] Morien, P.-L. (1998), The Hölder and the Besov regularity of the density for the solution of a parabolic stochastic partial differential equation, Bernouilli, 5, 275-298.

[MT03] Mueller, C. And Tribe, R. (2002), Hitting properties of the random string, Electronic Journal of Probability, 7, 1-29.

[NP94] Nualart, D. and Pardoux, E. (1994), Markov field properties of solutions of white noise driven quasi-linear parabolic PDEs, Stochastics and Stochastics Reports, 48, 17-44.

[N95] Nualart, D. (1995), The Malliavin calculus and related topics, Springer-Verlag.

[N98] Nualart, D. (1998), Analysis on Wiener space and anticipating stochastic calculus, Ecole d'Eté de Probabilités de Saint-Flour XXV, Lect. Notes in Math. 1690, Springer-Verlag, 123-227.

[S05] SAnz-SolÉ, M. (2005), Malliavin calculus with applications to stochastic partial differential equations, EPFL Press.

[W86] Walsh, J.B. (1986), An Introduction to Stochastic Partial Differential Equations, Ecole d'Eté de Probabilités de Saint-Flour XIV, Lect. Notes in Math., 1180, Springer-Verlag, 266-437.

[W84] Watanabe, S. (1984), Lectures on Stochastic Differential Equations and Malliavin Calculus, Tata Institute of Fundamental Research Lectures on Math. and Physics, 73, Springer-Verlag, Berlin. 